Florida International University FIU Digital Commons

$12-18-2013$

\title{
Perceptions and Experiences of Intimate Partner Violence Among Hispanic College Students
}

Racquel Vera

Florida International University, rvera006@fiu.edu

DOI: $10.25148 /$ etd.FI14040801

Follow this and additional works at: https://digitalcommons.fiu.edu/etd

Part of the Counseling Commons, Developmental Psychology Commons, Family Practice Nursing Commons, Other Nursing Commons, Pediatric Nursing Commons, and the Public Health and Community Nursing Commons

\section{Recommended Citation}

Vera, Racquel, "Perceptions and Experiences of Intimate Partner Violence Among Hispanic College Students" (2013). FIU Electronic Theses and Dissertations. 1172.

https://digitalcommons.fiu.edu/etd/1172 


\title{
FLORIDA INTERNATIONAL UNIVERSITY \\ Miami, Florida
}

\section{PERCEPTIONS AND EXPERIENCES OF INTIMATE PARTNER VIOLENCE AMONG HISPANIC COLLEGE STUDENTS}

\author{
A dissertation submitted in partial fulfillment of the \\ requirements for the degree of \\ DOCTOR OF PHILOSOPHY \\ in \\ NURSING \\ by \\ Racquel Vera
}

2014 
To: Dean Ora L. Strickland

College of Nursing and Health Sciences

This dissertation, written by Racquel Vera, and entitled Perceptions and Experiences of Intimate Partner Violence Among Hispanic College Students, having been approved in respect to style and intellectual content, is referred to you for judgment.

We have read this dissertation and recommend that it be approved.

$\begin{array}{r}\text { Frank Dillon } \\ \hline \text { Sandra Gracia Jones } \\ \hline \text { Larol A. Patsdaughter, Co-Major Professor } \\ \hline \text { Anahid Kulwicki, Co-Major Professor }\end{array}$

Date of Defense: December 18, 2013

The dissertation of Racquel Vera is approved.

Dean Ora L. Strickland

College of Nursing and Health Sciences

Dean Lakshmi N. Reddi

University Graduate School

Florida International University, 2014 
C Copyright 2014 by Racquel Vera

All rights reserved. 


\section{ACKNOWLEDGMENTS}

I would like to thank all of the people who have encouraged and supported me throughout the pursuit of my personal and professional endeavors.

Firstly, to Dr. Dorothy Brooten, Dr. Kevin O'Shea and Dr. Lidia Kos, my deepest gratitude for your much needed guidance as I reach the most coveted finish-line.

Second, I would like to thank the committee's Co-Chair Dr. Carol Patsdaughter, for keeping me focused and appreciate the concept of logical and mindful thinking (i.e., orders of orange, apples, grapes, and bananas). You will be greatly missed Dr. Pat. I never really referred you as Dr. Pat as you always asked me to. You were my teacher and will always be one of the best catalysts in life. My committee members, Dr. Sande Jones, Dr. Lidia Kos and Dr. Frank Dillon for your patience and kindness, for keeping me grounded with your comments and encouragements on my dissertation. Thank you for being members of my dissertation committee and also for making this journey attainable.

Also, I thank Cari Romeo, for your assistance in data collection and entry. I wish you all that are good and joyful. Shawn Muhammed and Rey Llanes for assisting me with data collection, you made this process very enjoyable. Ms. Karen Iglesia, I will never forget your encouraging words and caring actions. You were there to guide me through the labyrinth of the final lap of this journey.

Third, the completion of this project would not have been possible without my children's love, support, and admiration. To my daughter, Amanda Elizabeth, and son David Michael, I thank you for letting mommy study and hopefully win the contest when I was busy writing my dissertation. I know you both wanted to hang out and watch Glee yet you keep expressing your love for me even when I did not have time to give you 
much attention. I would like to thank Mr. Scott McLean for all the love, caring, and words of encouragements. To my mother, Luzviminda Vera Torres, you are always there for me as my inspiration while I was consumed for countless hours writing my dissertation. To my beautiful brothers and sisters, I love you so very much.

Fourth, to my dear colleagues and friends Priscilla, Betsy, Marie, Kelly and Dr. Janyce Dyer (You'll always be my teacher, I cannot do the first name basis), Suha and Carmen - I thank you for being my cheerleaders during every step of this journey. I thank you all from the bottom of my heart for being there for me anytime that I needed you. I will always cherish the relationship we shared as colleagues and friends. Betsy, I promise you that your investment will definitely yield a successful return.

Finally, to my students at South University, I would like to thank you for showing interest in my research project and for being proud of my educational accomplishment. 


\title{
ABSTRACT OF THE DISSERTATION
}

\section{PERCEPTIONS AND EXPERIENCES OF INTIMATE PARTNER VIOLENCE AMONG HISPANIC COLLEGE STUDENTS}

\author{
by \\ Racquel Vera \\ Florida International University, 2014 \\ Miami, Florida \\ Anahid Kulwicki, Co-Major Professor \\ Carol A. Patsdaughter, Co-Major Professor
}

Intimate partner violence (IPV) is recognized as a serious, growing problem on college campuses. IPV rates among college students exceed estimates reported for the general population. Few studies have examined the impact of IPV among the Hispanic college student (HCS) population or explored how HCSs perceive and experience IPV. Focusing on young adults (ages 18 to 25 years), this mixed methods study was designed to explore the perceptions and experiences of IPV focusing on levels of victimization and perpetration in relation to gender role attitudes and beliefs, exposure to parental IPV, acculturation, and religiosity. A sample of 120 HCSs was recruited from two south Florida universities. A subsample of 20 participants was randomly selected to provide qualitative responses. All participants completed a series of questionnaires including a demographic survey, the FPB, CTS2-CA, SASH, ERS and CTS2. Bivariate correlational techniques and multiple regressions were used to analyze data. Marked discrepancy between participants' perceived experience of IPV $(N=120)$ and their CTS2 responses $(n=116,96.7 \%)$. Only $5 \%$ of the participants saw themselves as 
victims or perpetrators of IPV, yet $66 \%$ were victims or $67 \%$ were perpetrators of verbal aggression; and $31 \%$ were victims or $32.5 \%$ were perpetrators of sexual coercion based on their CTS2 scores. Qualitative responses elicited from the subsample of 20 students provided some insight regarding this disparity.

There was rejection of traditional stratified gender roles. Few participants indicated that they were religious $(20.8 \%, n=25)$. Evidence for the theory of intergenerational transmission of violence was noted. Recall of parental IPV was a significant predictor of level of IPV victimization $(\beta=0.177, S E=0.85, p=0.041)$. Nursing and social service providers must be cognizant that contributing factors to either victimization and/or perpetration of IPV among college students must be addressed first (i.e., perceptions of IPV), both in acute (i.e., emergency department) and community (i.e., college and university) settings for optimum intervention outcome. 


\section{TABLE OF CONTENTS}

CHAPTER

PAGE

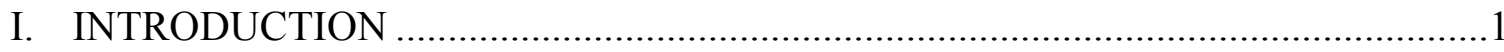

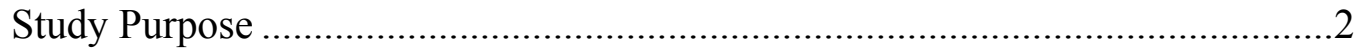

Significance of the Study .............................................................................

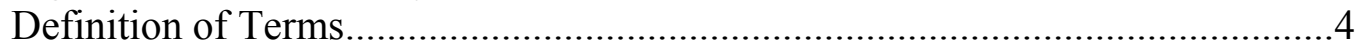

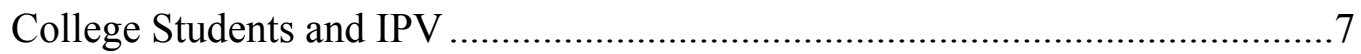

Hispanic College Students ........................................................................

Gender Symmetry, IPV, and Hispanic College Students ...........................10

Acculturation....................................................................................

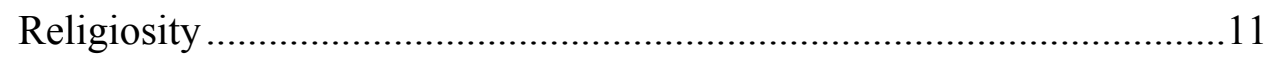

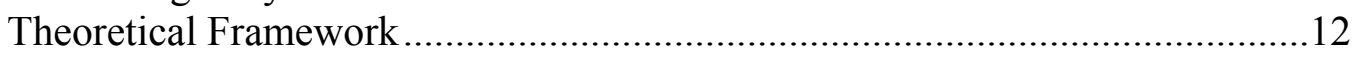

Intergenerational Transmission of Violence .............................................12

Research Questions and Hypotheses ..............................................................14

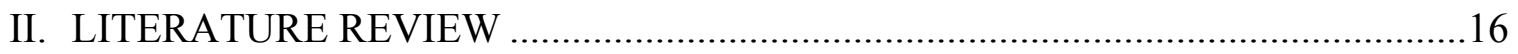

Factors Influencing IPV and the IGTV Among Hispanic Young Adults...........19

Adolescent Aggression ...........................................................................19

Traditional Latin Gender Role Ideology ................................................21

Marianismo and Domestic Violence..............................................22

Gender Role Socialization ..............................................................23

Changing Attitudes and Perspectives.................................................25

Gender Role Attitudes and Aggression............................................................2

Male Gender Role ............................................................................28

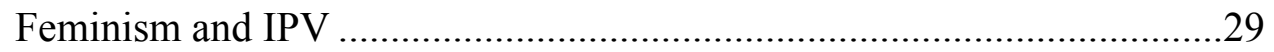

Family Violence....................................................................................

Intergenerational Transmission of Violence.........................................................33

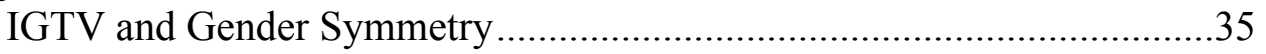

Acculturation..........................................................................................36

Prevalence of Partner Violence Among Hispanic People.....................................37

Attitudes Toward Partner Violence: Honor Cultures............................................39

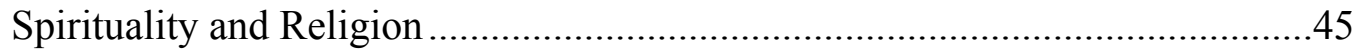

Religious Leaders' Beliefs...................................................................45

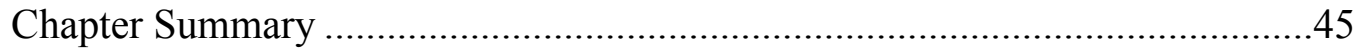

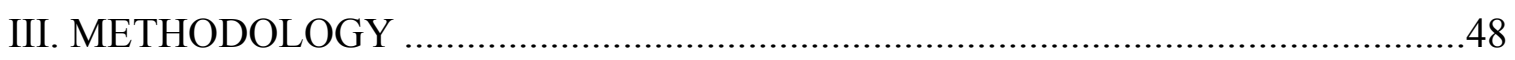

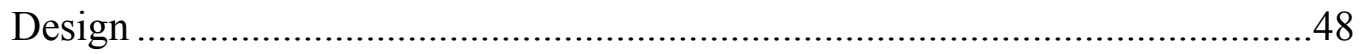

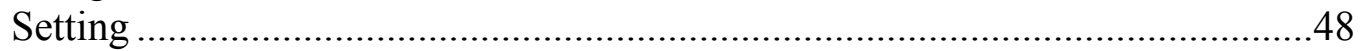

Sample

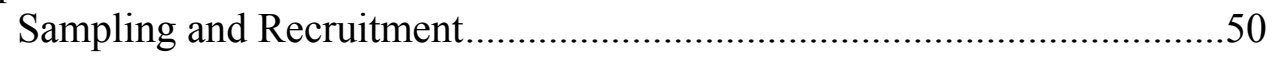

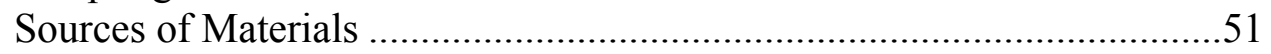

Quantitative Analysis ...........................................................................52

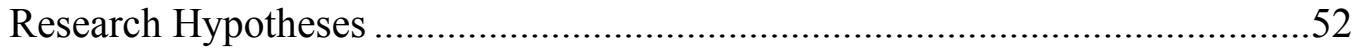




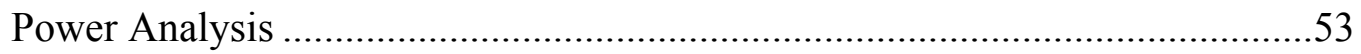

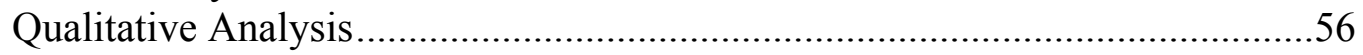

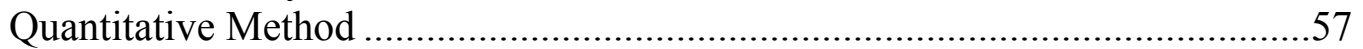

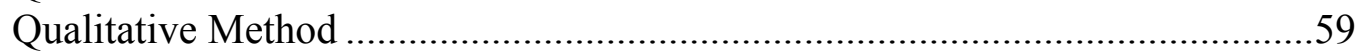

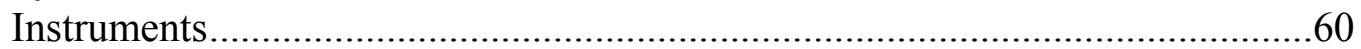

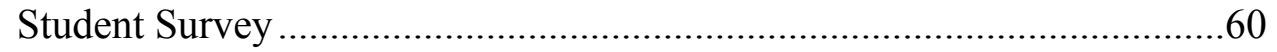

Overview of Study Measures................................................................60

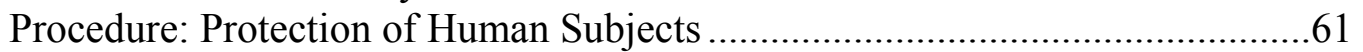

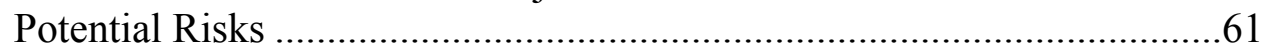

Adequacy of Protection Against Risks ......................................................61

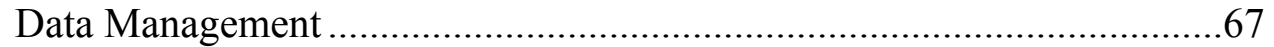

Recruitment and Informed Consent .........................................................67

Potential Benefits of the Research to Human Subjects and Others .....................69

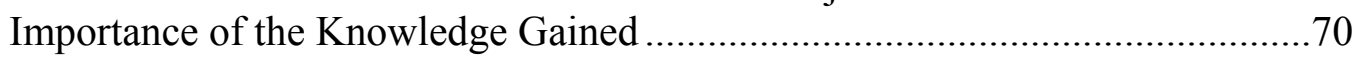

Inclusion of Women and Minorities ................................................................

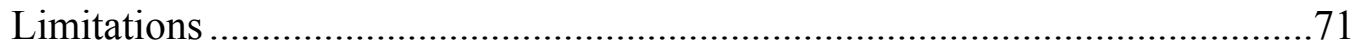

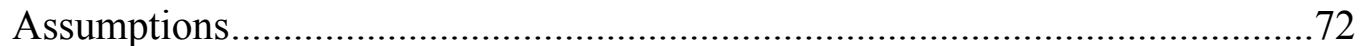

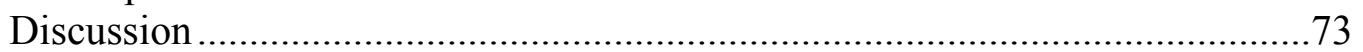

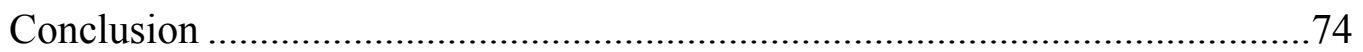

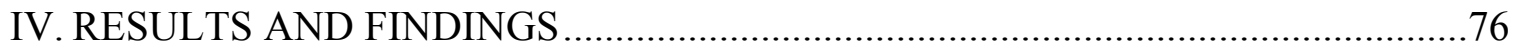

Demographic and Background Characteristics.................................................76

Reliability Estimates for Instruments............................................................77

Descriptive Findings on Major Study Variables................................................ 81

Cultural Gender Role .........................................................................82

Adult Recall of Parental IPV ................................................................83

Short Acculturation Scale for Hispanics...................................................84

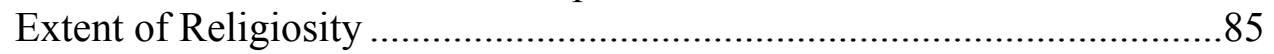

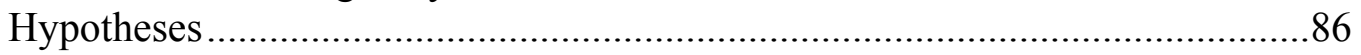

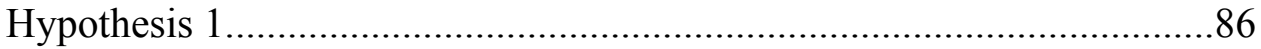

Gender and Perceptions of IPV....................................................... 86

Cultural Gender Roles and Perceptions of IPV ...............................87

Adult Recall of Parental IPV and Perceptions of IPV ......................88

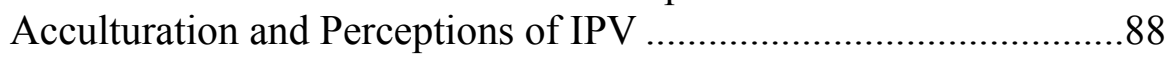

Religiosity and Perceptions of IPV ……….....................................89

Level of Victimization and Level of Perpetration and

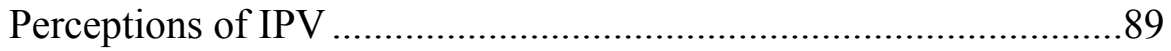

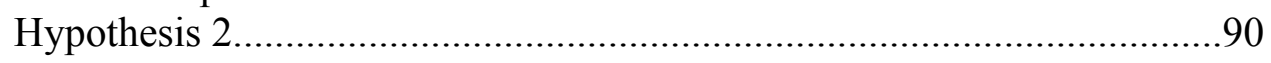

Gender and Experiences (Level) of Victimization ...........................91

Cultural Gender Role and Level of Victimization.............................91

Adult Recall of Parental IPV and Level of Victimization ................91

Acculturation and Level of Victimization ......................................92

Religiosity and Level of Victimization..........................................92

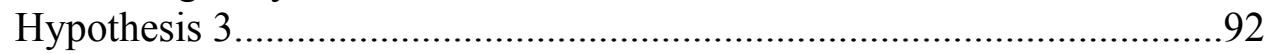

Gender and Experiences (Level) of Perpetration...............................93 
Cultural Gender Role and Level of Perpetration

Adult Recall of Parental IPV and Level of Perpetration..................94

Acculturation and Level of Perpetration...........................................94

Religiosity and Level of Perpetration ............................................94

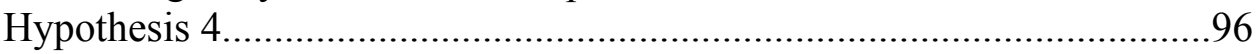

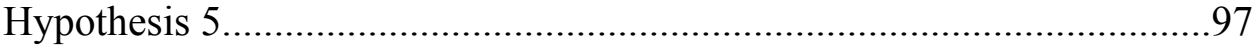

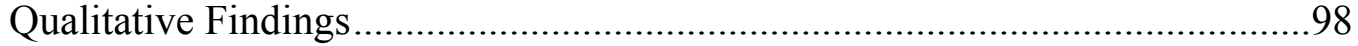

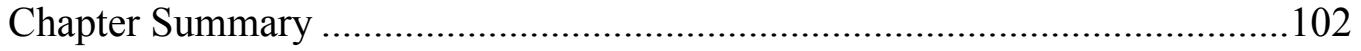

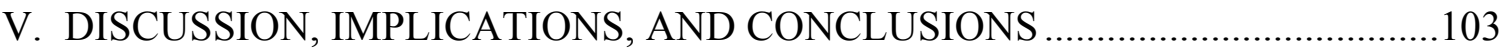

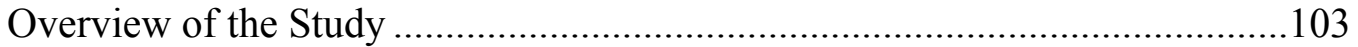

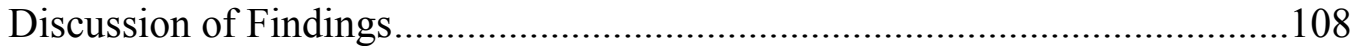

IPV Victimization and Perpetration: Subjective Perceptions Versus

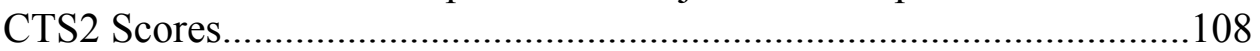

HCSs' Perceptions of IPV ...........................................................110

Student Participants' of IPV Versus Parental Perceptions ......................112

Types of Interpersonal Violence Identified by HCSs ...........................115

Cultural Gender Roles, Parental IPV, Acculturation, and Religiosity.....116

Cultural Gender Roles................................................................117

Experience of Parental IPV .......................................................119

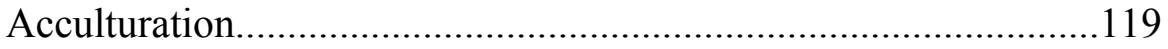

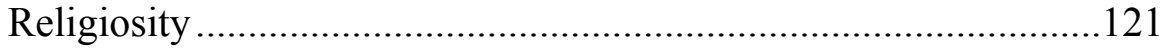

Relationships Among Gender, Cultural Gender Roles, Parental IPV,

Acculturation, Religiosity, and IPV Victimization..............................121

Relationships Among Gender, Cultural Gender Roles, Parental IPV,

Acculturation, Religiosity, and IPV Perpetration .................................122

Relative Contributions of Gender, Cultural Gender Roles, Parental IPV,

Acculturation, Religiosity, to IPV Victimization ..................................122

Relative Contributions of Gender, Cultural Gender Roles, Parental IPV,

Acculturation, Religiosity, to IPV Perpetration .....................................123

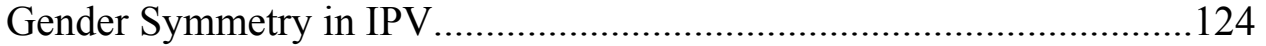

Cultural Gender Role Differences in IPV ............................................126

Intergenerational Transmission of Violence ...............................................128

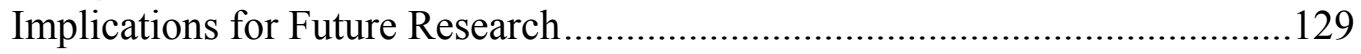

Implications for Nursing and Interdisciplinary Practice ................................132

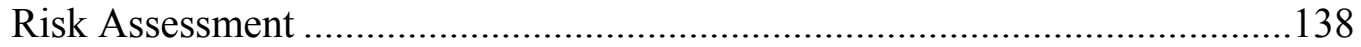

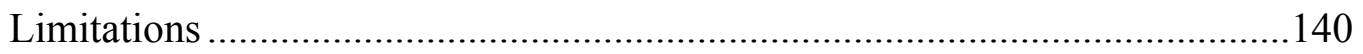

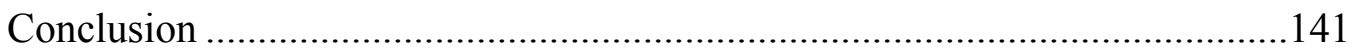

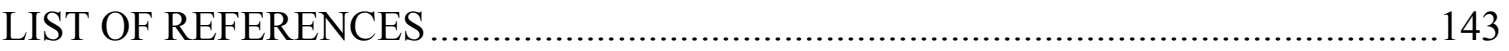

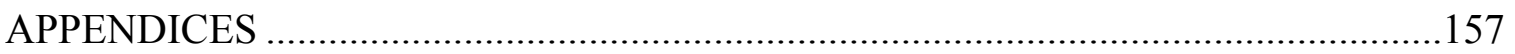

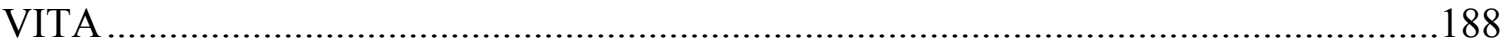




\section{LIST OF TABLES}

TABLE

PAGE

1. Test of Normality for Cultural Gender Roles, Adult Recall of Parental IPV, Acculturation, Religiosity, Past and Current Experiences of IPV, Level of Victimization and Level of Perpetration

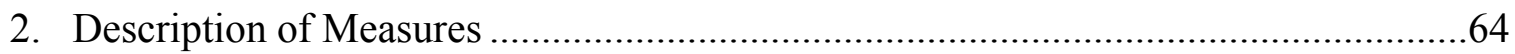

3. Demographic and Background Characteristics....................................................79

4. Cronbach Alphas for Cultural Gender Roles, Adult Recall of Parental IPV, Acculturation, Religiosity, and Past and Current Experiences of IPV Scales

5. Descriptive Finding on Major Study Variables

6. Descriptive Finding on Dependent Variables: Perceived Victimization and Perceived Perpetration

7. Mean Gender Role Ratings of Male and Female Hispanic College Students .84

8. Preferred Ethnicity of Social Groups

9. Correlations Between Cultural Gender Roles, Adult Recall Of Parental IPV, Acculturation, and Religiosity Scales, and Level of Victimization

10. Correlations Between Cultural Gender Roles, Adult Recall Of Parental IPV, Acculturation, and Religiosity Scales, and and Level of Perpetration

11. Regression Analysis of Level of Victimization Predicted by Gender, Cultural Gender Roles, Adult Recall of Parental IPV, Acculturation, and Religiosity

12. Regression Analysis on Level of Perpetration Predicted by Gender, Cultural Gender Roles, Adult Recall of Parental IPV, Acculturation, and Religiosity 


\section{ABBREVIATIONS AND ACRONYMS}

CDC Center for Disease Control and Prevention

CTS2 Revised Conflict Tactic Scale

CTS2-CA Conflict Tactic Scale - Parent Child

ERS Extent of Religiosity

FPB Familial Patriarchal Beliefs

HCSs Hispanic College Students

IGTV Intergenerational Transmission of Violence

IPV Intimate Partner Violence

IPVAC Intimate Partner Violence Assistance Clinic

NCIPC National Center for Injury Prevention and Control

NIH National Institutes of Health

NIMHD National Institute of Minority Health and Health Disparities

OVW Office on Violence Against Women

PI Principal Investigator

PTSD Posttraumatic Stress Disorder

RA Research Assistant

SASH Short Acculturation Scale for Hispanics

SES Social Economic Status

USDOJ United States Department of Justice

WHO World Health Organization 


\section{CHAPTER I}

\section{INTRODUCTION}

More than one in three women (35.6\%) and more than one in four men $(28.5 \%)$ in the United States have experienced rape, physical violence, and/or stalking by an intimate partner in their lifetime (Black et al., 2011). As early as the 1980s (Makepeace, 1981), research has indicated that college students have a higher prevalence of intimate-partner violence (IPV) and are more likely to exhibit violent behaviors, compared to married couples (Stets \& Straus, 1992). Moreover, IPV in this population is widespread. Most undergraduate and graduate students are in the age groups at highest risk for IPV (Coker, Sanderson, Cantu, Huerta, \& Fadden, 2008; Ramirez, 2007; B. A. M. Smith, Thompson, Tomaka, \& Buchanan, 2006). Furthermore, dating violence is a common problem on college campuses (Wasserman, 2004).

IPV is a serious problem among college students. Approximately 20\% (Shook, Gerrity, Jurich, \& Segrist, 2000) to 50\% (Straus \& Ramirez, 2007) of violence is perpetrated against one's intimate partner; while an estimated $30 \%$ of college students physically assault their intimate partners (Straus, 2004). The National Intimate Partner and Sexual Violence Survey 2010 Summary Report indicated that almost 38\% of female victims were 18 to 24 years of age at the time of their first completed rape victimization. The report also cited that among this age group, $34 \%$ of women and $28 \%$ of men were stalked by either an acquaintance, current partner, or former intimate partner. Among women who ever experienced rape, physical violence, and/or stalking by an intimate partner, nearly half (47.1\%) were between 18 and 24 years of age; while among men who 
ever experienced rape, physical violence, and/or stalking by an intimate partner, $38.6 \%$ were between the ages of 18 and 24 years (Black et al., 2011).

According to the 2009 American Community Survey 1-Year Estimates, Latinos constitute $15.8 \%$ of the U.S. population and are the largest and fastest growing minority group in the country (U.S. Census Bureau, 2010b.). In 2008, nearly two-thirds of Hispanic people in the United States self-identified as being of Mexican origin. Nine of the other 10 largest Hispanic origin groups-Puerto Rican, Cuban, Salvadoran, Dominican, Guatemalan, Colombian, Honduran, Ecuadorian and Peruvian—accounted for about a quarter of the U.S. Hispanic population (Dockterman, 2011).

\section{Study Purpose}

This dissertation project was a mixed-method study of IPV as seen through the eyes of young Hispanic adults aged 18 to 25 years. The success of prevention and intervention efforts in the area of IPV is contingent on understanding the intricate array of factors underlying the problem. A starting point for understanding the problem is having a coherent definition of IPV, specifically exploring Hispanic college student (HCS) perceptions and experiences of IPV.

\section{Significance of the Study}

Very few studies have explored the impact of IPV among the HCS population (Sanderson, Coker, Roberts, Tortolero, \& Reininger, 2004), including how they perceive and experience IPV. Focusing on emerging adults who are beginning to form intimate relationships is integral to understanding their dating relationships, gender-role socialization, abusive beliefs, attitudes, and behavioral patterns of abusive interactions (Magdol, Moffitt, Caspi, \& Silva, 1998). This study explored the perceptions and 
experiences of IPV including physical, emotional, and sexual violence among Hispanic/Latino youth, and other factors (e.g., exposure to familial violence, acculturation, and religiosity) among HCS that may contribute to or mitigate the incidence of IPV among HCSs.

A growing challenge noted in most recent research has suggested that incidences of IPV increase during youth and young adulthood (Noonan \& Charles, 2009). Actions that are highly correlated with IPV events are risky behaviors such as engaging in highrisk sexual behaviors, tobacco and illicit drug use, drinking and driving, alcohol abuse (T. A. Roberts, Auinger, \& Klein, 2005), ineffective social skills, and inability to manage anger (Foshee et al., 2008). Furthermore, a recent body of research on family violence has suggested that experiencing violence is associated with dating relationships that put young individuals "at risk for continuous dating behavior within and across relationships" (Noonan \& Charles, 2009, p. 1088).

Cuevas, Sabina, and Picard (2010) calculated that studies focusing on Latinos represented about a scant $1 \%$ of the research on victimization. As previously noted, Latinos constitute almost $16 \%$ of the U.S. population and is the largest and fastest growing minority group in the country (U.S. Census Bureau, 2010b). In response to the glaring knowledge gap as well as the methodological limitations of many studies (e.g., small sample size, reliance on convenience samples, focus on only one type of victimization), Cuevas et al. designed the Sexual Assault Among Latinas study to assess various types of interpersonal violence experienced by Latinas in the United States, along with psychological symptoms and their relationship to the experience of victimization. 
Thompson, Basile, Hertz, and Sitterle (2006) defined IPV as actual or threatened physical or sexual violence or psychological/emotional abuse. It includes threatened physical or sexual violence when the threat is used to control a person's actions. Various types of violence, whether physical, emotional, sexual, or even witnessing violence, may influence the growing child to believe that the violence is normal (Fagan, 2005). Common terms used to describe IPV are domestic abuse, spouse abuse, domestic violence, courtship violence, battering, marital rape, and date rape. The incidence of partner abuse varies based on different methods and definitions used to define the problem. Findings from multiple research studies have demonstrated that the cycle of abuse starts very early in life. Social-learning theory proposes that violence is a coping mechanism learned through observation or experience. Modeling is a contributory factor to learning violent behavior as well (Corvo, 2006; Fagan, 2005; Schwartz, Hage, Bush, \& Burns, 2006).

In general, the prevalence of IPV on college campuses makes it an important issue that merits greater research attention. With the increasing diversity of the U.S. college population, cross-cultural research would serve to illuminate differences and similarities across and within groups for the purpose of designing campus primary prevention and intervention campaigns.

\section{Definition of Terms}

The following terms used in this study are clarified to assist the reader's understanding: 
Acculturation. This is a process mandating that immigrants willingly modify their own culture as an accommodation to their transition to accepting the general values and attitudes of their new culture and homeland (Ryder, Alden, \& Paulhus, 2000).

Culture. Culture involves the shared perceptions, customs, traditions, values, beliefs and history among a group of people and provides a set of guidelines for a certain group of people to live by (Huff, 1999). It is "historically transmitted pattern of meaning embodied in symbols, a system of inherited conceptions expressed in symbolic forms by means of which communicate, perpetuate, and develop their knowledge about and attitudes toward life" (Geertz, 1973, p.89).

Ethnic identity. Ethnic identity is an individual's sense of self as a member of an ethnic group (Phinney, 2003).

Hispanic/Latino. The Merriam-Webster dictionary defined Hispanic as "being a person of Latin American descent living in the United States, especially of Cuban, Mexican or Puerto-Rican origin; while a Latino is a person of Latin American origin living in the United States" (Merriam-Webster, 2011a, 2011b). Individuals who indicated that they are "other Spanish/Hispanic/Latino" include those whose origins are from Spain, the Spanish-speaking countries of Central or South America, the Dominican Republic, or people identifying themselves generally as Spanish, Spanish American, Hispanic, Hispano, Latino, and so forth. Origin can be viewed as the heritage, nationality group, lineage, or country of birth of the person or the person's parents or ancestors before their arrival in the United States. People who identify their origin as Spanish, Hispanic, or Latino may be of any race (U.S. Census Bureau, 2010a.) 
Intergenerational transmission of violence. This is based on the original term coined by Bandura (1977) in social-learning theory that subsequently became socialcognitive theory (Bandura, 1986). It has been speculated that violent behavior is learned and adaptive and that in "social situations is most important in determining the frequency, form, circumstances, and target of the action. ... The acquisition of aggressive behavior can be learned through modeling or observational learning or by direct experience or practice" (Humphreys \& Campbell, 2011, p. 42).

Intimate partner violence (IPV). For the purposes of this dissertation, IPV will be based on the Centers for Disease Control and Prevention's (CDC, 2006) definition of IPV, which is actual or threatened physical or sexual violence or psychological/emotional abuse. It includes threatened physical or sexual violence when the threat is used to control a person's actions.

Patriarchy. Patriarchy is defined as social organization marked by the supremacy of the father in the clan or family, the legal dependence of wives and children, and the reckoning of descent and inheritance in the male line; control of men by a disproportionately large share of power (Merriam-Webster, 2011c).

Perpetrate. To perpetrate is to commit a crime or a violent or harmful act (Cambridge Dictionary Online, 2012a).

Religiosity. Religiosity is a process of searching that manifests itself in external rituals of devotion or worship. It operates on health by way of participation in institutionalized rituals and the fellowship of the faith-based community that shares the religion (Daly, 2005, p. 1238). It is also "an individual's beliefs and behavior in relation to the supernatural and/or high-intensity values" (Roof, 1979, p. 18). 
Victimize. To victimize is to treat someone in an intentionally unfair way, especially because of their race, sex, or beliefs (Cambridge Dictionary Online, 2012b).

\section{College Students and IPV}

Researchers and practitioners are alarmed by the high proportion of college students who consider some degree of physical violence in dating relationships "acceptable or normal in some circumstances" (emphasis in original, Wasserman, 2004). Dating violence encompasses physical violence, sexual violence, and stalking, which are often combined. Definitions of IPV include psychological abuse, which is more insidious and can be even more detrimental than physical abuse (World Health Organization [WHO], 2005). Of all forms of interpersonal violence, stalking may actually have the most damaging psychological effects (Cuevas et al., 2010).

Hispanic college students. Coker et al. (2008) investigated the prevalence of IPV in a sample of Mexican American women who attended a college located close to the Texas-Mexico border. Ranging in age from 18 to 35 years, a total of 149 women completed the survey. First-year students comprised the largest segment of the sample (34.5\%), followed by juniors (22.3\%), sophomores, (20.3\%), seniors (13.5\%), and graduate students $(6.5 \%)$. More than half the students resided with a parent $(60.2 \%)$ and a similar proportion (60\%) were single. Most of the students reported low or lower middle-class family incomes. The instruments used included the revised Conflict Tactic Scale (CTS), the Women's Experience With Battering scale, four items from the Sexual Experience Survey assessing sexual violence, four items from the National Violence Against Women Survey related to stalking, and a compressed version of the

Psychological Maltreatment of Women scale. The primary focus was on violence 
experienced within the last year, but Coker and colleagues added a question covering lifetime physical partner violence.

Of the total sample, $43 \%$ of the women experienced some form of partner violence, including sexual violence (12.1\%), physical violence (19.7\%), threats of physical violence (11.5\%), battering (15.6\%), stalking (19.7\%), and psychological abuse (30.2\%). A particularly unfortunate finding was that only one quarter of the women who experienced physical or sexual assault regarded violence as a problem in their relationship. However, Coker et al. (2008) noted that as the frequency of violence escalated, so did the probability that the women considered it problematic. There was also a substantial degree of co-occurrence of different types of violence. Among the 64 women who reported experiencing partner violence within the past year, $43 \%$ had been stalked by a partner and the vast majority (nearly 90\%) endured psychological abuse. Slightly more than half of the women (51\%) who experienced some type of violence were single.

While noting that these findings for the experience of violence by Mexican American college women do not diverge dramatically from empirical studies of dating violence, Coker et al. (2008) emphasized that they are nonetheless high. The incidence of stalking reported by Coker et al. is actually nearly twice as high as some studies of college students, but the concurrence of more than one type of violence is not unusual (Wasserman, 2004). Wasserman also noted that many students seemed to accept some degree of violence in relationships as "normal" or acceptable "in some circumstances" (emphasis in original, 2004, p. 19) and found these findings to be alarming. Tolerance of violence in dating relationships predisposes students to victimization by partners. 
The high incidence of partner violence, the prevalence of stalking (which can have serious detrimental effects on mental and physical health), and the disturbing number of women who seemed to tolerate moderate to severe physical violence in their relationships led Coker et al. (2008) to conclude that many college women are in dangerous relationships. The researchers noted that all the survey participants were informed of local community services for abused or battered women, and those who said they experienced IPV of any type were urged to avail themselves of appropriately targeted services. Coker et al. advocated future research into partner and dating violence with attention to cultural influences such as cultural heritage, acculturation, bicultural self-efficacy, and ethnic identity.

Daley and Noland (2001) explored IPV in an ethnically diverse sample of students attending a large community college using a modified version of the CTSRevised (CTS2). Women comprised roughly 53\% of the participants were $52 \%$ White, 20.3\% Hispanic, and $13.1 \%$ African American. Approximately two thirds of the women had been verbally abused by a dating partner within the last year. Women also made up the majority of students who had experienced some form of physical aggression, including, for the majority of victims, severe physical violence. A second study conducted online with university students focused on sexual victimization. The prevalence of rape among the respondents was much lower than CDC figures for the same year (i.e., $11.4 \%$ versus $20 \%$ ). Among the women who had been raped, $64 \%$ were between the ages of 17 and 24 the first time it happened, and $74 \%$ were in the same age group the last time it happened. 
Gender symmetry, IPV, and Hispanic college students. Drawing from the International Dating Violence Study, Straus and Ramirez (2007) examined gender symmetry in the prevalence, severity, and persistence of physical aggression against dating partners by university students in the United States and Mexico. Two sites with sociodemographically different student populations were chosen from each country. Across the four sites, there was strong evidence of gender symmetry. That is, men and women had similar prevalence rates for perpetrating acts of severe violence and for chronically perpetrating minor violence. Additionally, in the majority of couples with one violent partner, both partners had committed at least one act of violence. The one gender distinction that surfaced in the analysis was in the subgroup of students who committed acts of severe violence men in all four settings perpetrated severe violence more often than women. While concluding that these data affirm gender symmetry in dating violence, Straus and Ramirez acknowledged that women are more likely to incur serious injuries. The researchers emphasized the need for programs and policies targeting the primary prevention of partner violence by women to reduce partner victimization among both genders.

Acculturation. Ramirez (2007) investigated the relationship of acculturation and social integration to IPV perpetration in a sample of 348 Mexican American and White students recruited from two southwestern universities. The study was based on two theoretical perspectives of IPV: one, an ethnic perspective that there would be lower rates of IPV among Mexican American students, and second, Hirschi' s (1969) criminologicalsocial-control theory, which suggests IPV would be less common among students who were more socially integrated. In contrast to Ramirez's expectations for ethnicity, there 
were no significant differences in IPV between the two student groups. In fact, there was higher prevalence of IPV among Mexican American students compared to White students (i.e., $26 \%$ versus $18 \%$ ), but the difference did not reach statistical significance.

Acculturation was not a factor in the perpetration of IPV, although Ramirez (2007) acknowledged that the English-speaking, relatively high-income Mexican American university students comprised a sophisticated group with minimal differences in acculturation. Social integration, however, did affect the prevalence of IPV despite the fairly high levels of social integration found for the Mexican American and White students as a group. Specifically, higher levels of social integration were linked with lower prevalence of IPV, thus supporting the social-control theory of interpersonal violence.

Religiosity. Religiosity has been linked to IPV. Davidson, Moore, and Ullstrup (2004) studied college women's religiosity and sexual attitudes. In this study, the authors concluded that the higher the religiosity score, the less likely these women will engage in sexually risky behaviors such as low condom use and multiple sexual partners. Deviant behaviors, including perpetration of violence and alcohol consumption among college students have been linked to levels of religiosity (Cochran, Beeghley, \& Bock, 1988). Interestingly, Higginbotham, Ketring, Hibbert, Wright, and Guarino (2007) explored levels of religiosity among 18 - to 24 year-old women. Participants who reported experiencing low religiosity also reported more courtship violence compared to those who have high-religiosity experiences. It was also found that women who seek partners who have similar religious and spiritual values experience less violence. Future studies 
evaluating the effects of religiosity on courtship violence should include measures of relationship religiosity.

\section{Theoretical Framework}

Intergenerational transmission of violence. The theory of intergenerational transmission of violence (IGTV) is based on social-learning theory (Bandura, 1977). Observational learning (i.e., modeling) is a cornerstone of social-learning theory; thus, witnessing domestic violence even without being victimized can have a marked impact on later behavior. There is some controversy regarding the extent that the IGTV predicts the perpetuation of abuse, with estimates ranging from $18 \%$ to $70 \%$ (Allen, 2001). Citing researchers Kaufman and Zigler $(1987,1993)$ who concluded that a $30 \%$ intergenerational transmission rate constituted the "best estimate," Allen noted that while accounting for less than half of all individuals, a figure of $30 \%$ is "six times the base rate of abuse in the general population" (emphasis added, 2001, p. 63).

The WHO (2005) recognized prior victimization and family violence as major risk factors for IPV victimization. In many cases, family violence takes place in a constellation of factors that raise the risk of subsequent violence, such as poverty and related stressors and substance abuse. Culture and religion play powerful roles in the perpetuation of abuse. On the other hand, high self-esteem, social support, recognition of the damage caused by family violence, and deliberate planning strategies to protect against personal victimization (e.g., delaying marriage, pursuing education, achieving financial independence) foster resilience in women who have experienced childhood abuse and domestic violence (Belknap \& Cruz, 2007; DeJonghe, Bogat, Levendosky, \& 
von Eye, 2008; A. R. Roberts, 2006). Crane and Constantino (2003) advocated tailoring interventions for abused women to underpin their psychosocial and social support needs.

There is little dispute that understanding IPV is a complex endeavor. Conceptions of IPV and risk appraisal for future victimization can differ dramatically between clinicians and women who experience IPV (Cattaneo, 2007). Furthermore, professionals from different disciplines have different perspectives, and there are few clear guidelines for intervention within disciplines (Magnussen et al., 2004; Tower, 2003, 2006; Wandrei \& Rupert, 2000).

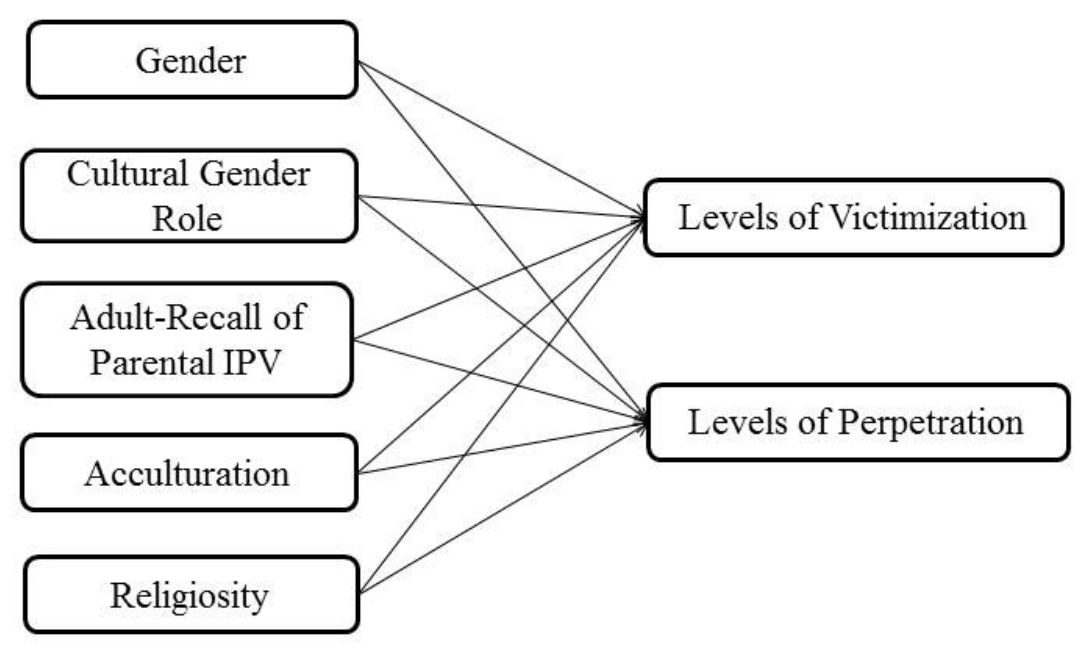

Figure 1. Author's conceptual framework for primers of intergenerational transmission of violence, based on Bandura's (1977) observational/modeling theory.

Independent variables represented factors at the sociocontextual level on how HCSs perceive and experience IPV. These are gender, cultural gender roles, adult recall of parental IPV, acculturation, and religiosity. The model hypothesizes possible relationships and relative contributions of the level of victimization and the level of perpetration among HCSs. The hypothesized direction of the arrows follow a regression 
pattern in the model to provide better understanding of the relationships between variables that are consistent with Bandura's (1977) observational/modeling theory. IGTV is widely considered from the perspective of social-learning theory, with the consequence that variables external to that perspective are often overlooked (Corvo, 2006). Although witnessing or experiencing abuse in the family of origin is well supported in the literature as a key component of the IGTV, there has been less attention to other family-of-origin factors that contribute to, mediate, or moderate future IPV. A focus on consequences of parenting on future intimate violence is needed beyond the effect of modeling abusive behavior (Schwartz et al., 2006).

\section{Research Questions and Hypotheses}

This dissertation project was a mixed-method study of IPV major concepts in the theoretical framework as seen through the eyes of young Hispanic adults. The success of prevention and intervention efforts in the area of IPV is contingent on understanding the intricate array of factors underlying the problem. A starting point for understanding the problem was having a coherent definition of IPV, specifically exploring HCS perceptions and experiences of IPV based on these following inquiries:

1. What are HCSs' perceptions of IPV?

2. What are the levels of (a) cultural gender roles; (b) adult recall of parental IPV; (c) acculturation; and (d) religiosity among HCSs?

3. Are there relationships between gender, cultural gender roles, adult recall of parental IPV, acculturation, religiosity, and level of victimization?

4. Are there relationships between gender, cultural gender roles, adult recall of parental IPV, acculturation, religiosity, and level of perpetration? 
5. What are the relative contributions of cultural gender roles, adult recall of parental IPV, acculturation, and religiosity to level of victimization?

6. What are the relative contributions of cultural gender roles, adult recall of parental IPV, acculturation, and religiosity to level of perpetration?

Research Question 1 was addressed through content and descriptive data analysis; while Research Questions 2 through 6 were addressed through quantitative analysis. Five hypotheses were tested:

$\mathrm{H}_{1}$ Perception of IPV is directly related to gender, cultural gender roles, adult recall of parental IPV, acculturation, religiosity, level of victimization, and level of perpetration.

$\mathrm{H}_{2}$ There is a relationship between gender, cultural gender roles, adult recall of parental IPV, acculturation, religiosity, and level of victimization.

$\mathrm{H}_{3}$ There is a relationship between gender, cultural gender roles, adult recall of parental IPV, acculturation, religiosity, and level of perpetration.

$\mathrm{H}_{4}$ There are relative contributions of cultural gender roles, adult recall of parental IPV, acculturation, and religiosity to level of victimization.

$\mathrm{H}_{5}$ There are relative contributions of cultural gender roles, adult recall of parental IPV, acculturation, and religiosity to level of perpetration. 


\section{CHAPTER II}

\section{LITERATURE REVIEW}

The literature presented in this review was drawn from PubMed and the following EBSCOhost databases: Academic Search Premier, MasterFILE Premier, PsycINFO, PsycARTICLES, and MEDLINE. Keywords used either individually or in conjunction included intimate partner violence, domestic violence, interpersonal violence, dating violence, abuse, aggression, attitudes, disclosure, college students, Latinas, Latinos, Hispanics, young adults, women, men, gender, culture, and ethnicity.

In searching the PsycINFO database using the keywords "Latin" and "victim" and "Hispanic" and "victim," compared to a broad search using only "victimization," Cuevas et al. (2010) calculated that studies focusing on Latinos represent a scant $1 \%$ of the research on victimization. For perspective, according to the 2009 American Community Survey 1-Year Estimates, Latinos constituted 15.8\% of the United States population and are the largest and fastest growing minority group in the country (U.S. Census Bureau, 2010a.). In 2008, nearly two-thirds of Hispanic people in the United States self-identified as being of Mexican origin. Nine of the other 10 largest Hispanic origin groups-Puerto Rican, Cuban, Salvadoran, Dominican, Guatemalan, Colombian, Honduran, Ecuadorian, and Peruvian - accounted for about a quarter of the U.S. Hispanic population (Dockterman, 2011; U.S. Census Bureau, 2010b ).

In response to the glaring knowledge gap as well as the methodological limitations of many studies (e.g., small sample size, reliance on convenience samples, focus on only one type of victimization), Cuevas et al. (2010) designed the Sexual Assault Among Latinas study to assess various types of interpersonal violence 
experienced by Latinas in the United States, along with psychological symptoms and their relationship to the experience of victimization.

Working from a similar interest in addressing research gaps in the knowledge and understanding of interpersonal violence, B. A. M. Smith and colleagues (2006) developed the Intimate Partner Violence Attitude Scales, which they tested in a sample of Mexican American college students. As motivation for their research, B. A. M. Smith et al. cited both the minimal research on Mexican Americans and the relative lack of attention to college students' attitudes toward IPV. College students are frequently used as research participants in evaluating the utility of a novel assessment tool. When the topic is interpersonal violence, the choice of a college student sample is particularly apt. Most undergraduate and graduate students are in the age groups at highest risk for IPV. B. A. M. Smith et al., along with Coker et al. (2008) and Ramirez (2007), who explored partner violence among Mexican American students, chose university students for that reason. Furthermore, dating violence is a common problem on college campuses (Wasserman, 2004).

Researchers and practitioners have been alarmed by the high proportion of college students who consider some degree of physical violence in dating relationships "acceptable or normal in some circumstances" (emphasis in original, Wasserman, 2003/2004). Dating violence encompasses physical violence, sexual violence, and stalking, which are often combined. Definitions of IPV include psychological abuse, which is more insidious and can be even more detrimental than physical abuse (WHO, 2005). Of all forms of interpersonal violence, stalking may actually have the most damaging psychological effects (Cuevas et al., 2010). 
Studies that have examined the incidence and prevalence of IPV among Latinas compared to other ethnic groups have tended to produce inconsistent results (GonzalezGuarda, Peragallo, Vasquez, Urrutia, \& Mitrani, 2009). Some studies have reported equivalent rates of IPV victimization for Latina and non-Latina women (Bonomi, Anderson, Cannon, Siesnick, \& Rodriguez, 2008; Catalano, 2007), whereas other studies have found higher rates of victimization among Latinas (Cuevas et al., 2010; Flake \& Forste, 2006; Hazen \& Soriano, 2005; Murdaugh, Hunt, Sowell, \& Santana, 2004). Underreporting is a persistent issue in understanding the incidence and prevalence of domestic violence. Linguistic and cultural barriers inhibit the disclosure of IPV by Latinas, thus signifying the need for culturally sensitive community services (MontalvoLiendo, 2009; Montalvo-Liendo, Wardell, Englebretson, \& Reininger, 2009; Rodriguez, Sheldon, Bauer, \& Perez-Stable, 2001).

Numerous researchers have implicated traditional Latin gender-role ideology, entrenched in the cultural constructs of machismo and marianismo, supporting male privilege and power and female self-sacrifice and submission as key contributors to the incidence of violence by men against women (Ahrens, Rios-Mandel, Isas, \& del CarmenLopez, 2010; Edelson, Hodoka, \& Ramos-Lira, 2007; Harris, Firestone, \& Vega, 2005; Lehrer, Lehrer, \& Zhao, 2010; Rondon, 2003). In particular, the acceptance of traditional gender roles leads to the acceptance of domestic violence and the willingness of abused Latinas to remain in abusive relationships (Kasturirangan \& Williams, 2003; Vandello, Cohen, Grandon, \& Franiuk, 2009). At the same time, Ahrens et al. (2010) questioned the extent to which Latin women and men in the United States actually adhere to traditional gender-role norms. Even women with fairly low levels of acculturation often embrace 
ideals of gender equality (Ramos-Lira, Koss, \& Russo, 1999). Factors such as acculturation, education, socioeconomic status (SES), country of origin, and religious beliefs as well as individual differences in attitudes, beliefs, and experiences must all be considered in understanding the issue of IPV.

Ahrens et al. (2010) made a valid point in cautioning against stereotypical assumptions that Hispanic men and women endorse traditional cultural gender roles and values. Nevertheless, there is empirical support, including evidence from their own study, that traditional gender-role ideology or "scripts" figure prominently in attitudes toward IPV by women and men of Latin heritage. The following section will provide a background on traditional Latin American gender roles and their potential influence on attitudes toward and acceptance of IPV.

\section{Factors Influencing IPV and the IGTV Among Hispanic Young Adults}

Adolescent aggression. Moretti, Obsuth, Odgers, and Reebye (2006) explored the relationship between exposures to IPV aggressive behavior in adolescents by including posttraumatic stress disorder (PTSD) as an important factor in the dynamics involved. Specifically, the researchers theorized that adolescents with PTSD would be more predisposed toward relationship violence. The study also examined the prospective divergent effects of witnessing violence perpetrated by fathers and mothers and the independent effects on each gender. The participants were 63 girls and 49 boys drawn from two referral sources in the greater Vancouver area, a provincial center for the assessment of severe behavior problems and juvenile-justice facilities. Two-thirds of the participants were Caucasian, 22\% were Aboriginal, and the remaining represented a variety of ethnic groups. The relatively high representation of youth from Aboriginal 
families is consistent with the high rates of domestic violence reported among Native Americans and Alaska Natives in the United States (Tjaden \& Thoennes, 2000). The overwhelming majority of participants (i.e., $89 \%$ of the girls and $92 \%$ of the boys) resided in two-parent families (Moretti et al., 2006). Nevertheless, the fact that roughly $10 \%$ of the adolescents did not add weight to the assertion that the U.S. data underestimate the number of children exposed to IPV (McDonald, Jouriles, RamisettyMikler, Caetano, \& Green, 2006).

Certain gender differences emerged from the analysis (Moretti et al., 2006). Boys who witnessed their fathers commit violent acts against their mothers were more predisposed to display physical aggression toward their friends than those who did not. In a parallel fashion, girls who saw their mothers commit acts of IPV were more inclined toward physical aggression toward their friends than their counterparts who did not. There were no cross-gender effects. However, witnessing physical aggression by the parent of the opposite gender had no effect on the actions of the boys or girls toward their friends. This supports the social-learning tenet that behavior modeling is most powerful when the model is someone with whom one can closely identify (Bandura, 1977).

An intriguing finding was that girls who saw their fathers commit acts of IPV were more likely to be physically aggressive toward their fathers (Moretti et al., 2006). There were no other associations between IPV and aggression toward parents. Noting that these findings contrasts with another study that reported that mothers were more often the victims of aggressive behavior by their children, Moretti et al. (2006) pointed out that the earlier study covered a broad range of children whereas their study focused entirely on high-risk adolescents. They suggested that adolescents might be more 
provoked to aggression by witnessing their fathers commit IPV. In a study conducted in United Kingdom, children of all ages reported intervening to prevent their mothers from being victimized (Leason, 2005).

Another unusual finding was that witnessing their mothers committing IPV was associated with relationship aggression for both boys and girls, while fathers' violent behavior was not (Moretti et al., 2006). Although there could be several explanations for this effect, Moretti et al. (2006) found the most plausible that relationship violence emanated from being a victim rather than a witness of physical abuse. The results confirmed the association between family violence and child abuse. Roughly $60 \%$ of the adolescents exposed to IPV were physically abused by one or both parents.

The incidence of PTSD was high: about one-third of the adolescents met Diagnostic and Statistical Manual of Mental Disorders, 4th edition (APA, 2000) criteria for PTSD (Moretti et al., 2006). More than twice the proportions of girls to boys were affected (i.e., $46 \%$ versus 22\%). Moretti et al. (2006) proposed a developmental model of trauma in which PTSD is the central facet in understanding the impact of family violence on children and youth. They called for future research to explore the unique ways that paternal versus maternal IPV affects the development of girls and boys with the goal of neutralizing the intergenerational transmission of aggressive and violent behavior.

Traditional Latin gender-role ideology. The culture of machismo promotes male dominance and privilege. Marianismo is based on the premise that women model themselves after the Virgin Mary, a paradigm of self-sacrifice. Mary is considered to be spiritually and morally superior by virtue of putting the needs of others first and being capable of enduring suffering (Bracero, 1998; Rondon, 2003). Submissiveness, strength 
in adversity, and personal sacrifice for husband and children are the qualities of a "good" woman. Placed in the context of domestic violence, "Latina women are strong and brave because they can put up with abuse" (Kasturirangan \& Williams, 2003, p. 173).

Edelson and colleague (2007) argued that marianismo, machismo, and familismo (i.e., strong family ties that subordinate individual interests to the collective good of the family) keeps many Latina women trapped in abusive relationships, diminishes their coping resources, and intensifies psychological distress. Rondon (2003) viewed marianismo as a distortion of the Virgin Mary that promotes female passivity and patriarchal violence in Latin American cultures. Another related concept is respeto, respect for authority, which underlies the high power distance found in Latin cultures (Bracero, 1998). Familismo and respeto present especially formidable obstacles to acknowledging child sexual abuse committed by a family member or a respected authority figure such as a teacher or priest (Ramos-Lira et al., 1999).

Marianismo and domestic violence. Based on their work with Latina domestic violence survivors, Kasturirangan and Williams (2003) observed two conceptions of marianismo. The first is summed up by the comment of one woman that "las mujeres latinas de nuestra cultura somos educadas para servir al hombre" (Latina women of our culture are educated to serve men" p. 169). In accordance with the principles of marianismo, they described the "typical" Latina as a woman who is submissive and sacrifices her own needs for the sake of her husband and family. However, rather than regarding a woman as being weak for tolerating abuse, they viewed her as being strong and courageous for being able to endure abuse. Women were described as strong, hard- 
working, and responsible, whereas only one woman in the study described men as responsible or in any positive way.

According to Kasturirangan and Williams (2003), the ability to derive pride in suffering represents a positive facet of marianismo that counselors should attempt to understand. They also viewed strong family bonds as a resource for abused women as opposed to an obstacle to leaving an abusive relationship. However, while virtually all sources have agreed that programs and services for the prevention and intervention of domestic violence should be culturally sensitive, most decry marianismo and familismo for restricting the lives of women and making them vulnerable to IPV (Edelson et al., 2007; Rondon, 2003). There is far more evidence that women remain in abusive relationships out of fear and lack of resources that would allow them to leave than out of a sense of pride in enduring suffering (Edelson et al., 2007; Gonzalez-Guarda et al., 2009; Montalvo-Liendo et al., 2009; Murdaugh et al., 2004; Rodriguez et al., 2001). Shame, depression, and low-self-esteem are extremely prevalent among abused Latinas and they have the insidious effect of making it more difficult for women to disclose the abuse and leave the abuser.

Gender-role socialization. Several of the Latina abuse survivors commented that the belief that women should cater to men and be submissive and self-sacrificing was being challenged by women who were more educated and independent (Kasturirangan \& Williams, 2003). Personal attitudes toward traditional gender scripts vary tremendously among individuals in the same cultural group (Vandello \& Cohen, 2003). Raffaelli and Ontai (2004) explored the transmission of traditional cultural gender values in Latin families in two studies that examined how parents socialize their children. The first study 
focused on the family-socialization experiences of Latina women between the ages of 20 and 45 who had grown up in Spanish-speaking households. The 22 women were relatively well educated: all had graduated from high school, and half had a college or graduate degree or some college experience. In contrast, more than half of their mothers and fathers had not completed high school, and many parents had not gone beyond ninth grade.

Three dominant themes arose from the women's narratives of their experiences growing up: different treatment for girls and boys, parents' enforcement of stereotypically feminine behavior for daughters, and the restriction of girls' activities outside the home (Raffaelli \& Ontai, 2004). There was no evidence that socialization toward the traditional feminine gender role was less stringent for younger women. The push toward traditional gender roles and curtailment of personal freedom intensified during adolescence. The themes that emerged from the study were used to create a survey instrument for a larger study of Latina/o college students. A total of 97 women and 69 men ranging in age from 19 to 45 (median age $=21$ years) was recruited from four Midwestern campuses (i.e., two public universities, a private university, and a community college).

All female participants reported that they had more restrictions placed on their activities than their male counterparts. Raffaelli and Ontai (2004) noted that male participants agreed that they had more freedom at home than their sisters or other female relatives, thus corroborating the women's reports of being restricted. The results also showed that mothers took a more direct role in the gender socialization of daughters, while the fathers assumed a more direct role in socializing their sons. The parents' own 
gender-role attitudes were the predominant factor in their gender-socialization practices. Demographic factors such as birthplace, language, and education were indirectly related through their influence on these attitudes. For daughters, traditional gender-role attitudes on the part of mothers and fathers translated into encouragement to adopt stereotypically feminine behavior. For sons, a mother's traditional gender-role attitudes resulted in encouragement to engage in traditionally masculine behavior. A father's egalitarian attitudes and use of English at home had some influence on the son's encouragement to do household chores.

Changing attitudes and perspectives. Ruiz-Balsara (2002) explored attitudes toward machismo (conceptualized as both a cultural construct and as negative masculinity) and marianismo (conceptualized as submission) by Hispanic adults, with emphasis on the influences of gender, education, acculturation, SES, and religious beliefs. The study also examined the relationships between familism and machismo and marianismo. The findings revealed significant differences in the endorsement of the cultural construct of machismo across all variables examined with the exception of religion. As Ruiz-Balsara anticipated, the results showed stronger support for machismo among men and less-educated and less-acculturated respondents. Familism was moderately associated with both facets of machismo in opposite directions.

A study that explored what Latina/o women and men would like to see in a marriage-education program revealed that domestic violence and conflict-resolution skills were important topics and also that both women and men had fluid ideas of family gender roles. The study was conducted with a diverse sample of adults living in a Western community who were divided into homogenous focus groups: high school women, high 
school men, young adult single women, young adult single men, women in committed relationships, men in committed relationships, women making the transition to parenthood, men making the transition to parenthood, married women with children, married men with children, and professionals (Snyder, Duncan, \& Larson, 2010). Most of the participants were Mexican American, with some participants from South and Central America.

Domestic violence was a prominent concern for the single women, and some explicitly expressed concern over marrying someone who might be physically violent (Snyder et al., 2010). The women who were becoming parents were also concerned about community resources to protect against family abuse. Challenging the notion of traditional gender roles, the prospective mothers desired equal relationships in which their partners shared activities such as cooking and changing diapers, and most of the males, including high school students, single men as well as prospective fathers, wanted parenting classes. Across all groups, poor communication, infidelity, and finances were implicated as the main causes of marital problems, while good communication skills emerged as a key resource for strengthening relationship bonds.

Interestingly, conflict-resolution skills surfaced as a major topic in the men's groups but not in the women's groups (Snyder et al., 2010). It is possible that the men recognized conflict-resolution skills as a proactive strategy for averting physical or psychological abuse. Unique themes among the professionals included anger management, self-esteem issues, and decision-making skills. In view of the traditional Latin gender roles, Snyder et al. did not find it unexpected that infidelity (accepted for men but condemned in women), communication skills, and equal partnerships should be 
mentioned as important topics for community education. The professionals raised the issue of domestic-violence awareness campaigns, including information on legal ramifications in the United States.

Snyder et al. (2010) agreed with Ahrens et al. (2010) that there are numerous individual variations to the extent that men and women of Latin heritage adhere to traditional gender roles, pointing out that many Latino couples' relationships, regardless of their national origin, depart from traditional gender roles. Both research teams emphasized the importance of being sensitive to individual differences as well as cultural concepts on issues related to gender roles and relationships. There was a virtually unanimous preference by participants in the marriage-education study to have leaders and facilitators of Latin ethnicity (Snyder et al., 2010).

\section{Gender-Role Attitudes and Aggression}

According to Rondon (2003), domestic violence in patriarchal cultures is rooted in power dynamics that perpetuate gender inequities. The factors that promote and reinforce violence perpetrated by men include the desire to control women, emanating from insecurity; norms that accept male dominance over women; power differential between males and females; and the predisposition of certain groups to rely on violence as a means of communication. From the victim's standpoints, factors contributing to the perpetuation of violence include the perception that violence is a "normal" part of a woman's life, submissive and passive attitudes, and a strong commitment to the relationship as the central facet of a woman's life. 
Male gender role. Rondon's (2003) specific focus has been Latin America, which has unduly high rates of domestic violence. In addition to gender inequities, factors such as economic stress and political and social upheaval contribute to the prevalence of domestic violence in certain countries (Flake \& Forste, 2006; WHO, 2005). However, adherence to traditional concepts of masculinity has been associated with partner violence regardless of cultural heritage. In a review of the literature on masculinity and partner violence, Moore and Stuart (2005) found that the relationship depended on how masculinity is operationalized. About half the studies used collegestudent samples. The overall findings suggested that men may resort to violence when they feel their masculinity is threatened or feel they need to maintain male power, thus supporting the idea that the use of violence by men to control women comes from insecurity (Rondon, 2003).

According to Moore and Stuart (2005), men's beliefs regarding male gender-role expectations are the driving force in psychological aggression toward a relationship partner. Based on the research, Moore and Stuart concluded that men's "attitudes toward women's rights and roles are not as relevant to understanding violent behavior as how men respond to situations in which they feel challenged or threatened in conflicts with women" (2005, p. 56).

Jakupcak, Lisak, and Roemer (2002) examined the influence of masculine genderrole ideology and gender-role stress on the perpetration of partner violence in a sample of 165 college men. Masculine ideology per se was not significantly linked with aggression and violence. However, high levels of gender-role stress could provoke a violent response, particularly in conjunction with a high degree of masculine gender-role 
ideology. An interesting finding was that the combination of high masculine ideology and low gender-role stress decreased tendencies toward aggression and violence. Jakupcak et al. (2002) proposed that this effect might represent a "chivalrous subtype" of men with traditional masculine gender-role attitudes (p. 104). Furthermore, this "chivalrous" aspect of traditional masculinity is sometimes conceptualized as positive machismo whereas behavior that is controlling and violent represents negative machismo. Devotion and dedication to his children and to the women in his family are attributes of machismo, along with courage, strength, and indomitable will (Snyder et al., 2010). However, the allegedly positive chivalrous side of machismo carries negative implications for women because it implies that women are weak, vulnerable, and in need of protection (Bracero, 1998).

Feminism and IPV. Rondon's (2003) portrayal of domestic violence reflects the feminist theory of IPV, which asserts that IPV arises from patriarchal social structures that socialize males and females into stratified gender-specific roles (Próspero, 2008). Violence is seen as emanating from men's exercise of power to control women. From this perspective, the emphasis is on "the patriarchal family, the social construction of masculinity, and the structural factors that restrict a woman's ability to break away from IPV victimization" (p. 640). According to the feminist perspective, men comprise the vast majority of perpetrators of IPV with women as the victims (i.e., gender asymmetry of IPV).

Patriarchal values are central to feminist theories of domestic violence. Some researchers target "hostile masculinity," namely negative attitudes toward women and the acceptance of violence toward women as a major cause of such violence (Graham- 
Bermann \& Brescoll, 2000, p. 600). Others who have worked with male batterers have argued that some men invoke patriarchal beliefs to rationalize their actions only after committing abuse. Graham-Bermann and Brescoll (2000) investigated the associations between domestic violence and patriarchal-, family-, and gender-stereotyped attitudes by surveying children exposed to varying degrees of domestic violence. The participants were 21 children ranging in age from 6 to 12 years who responded to items related to stereotypes about power and violence in the family.

The analysis produced four major factors: male power, female power, violence privilege, and family autonomy (Graham-Bermann \& Brescoll, 2000). Boys expressed more stereotyped attitudes than girls, especially with regard to the male-power dimension. Younger children and children from lower income families held more stereotyped beliefs than their older and more affluent counterparts, a finding consistent with prior research. Especially notable was that children of ethnic-minority heritage endorsed more stratified family-role beliefs and more extreme attitudes regarding "the appropriateness and necessity of the use of physical violence in the family" (p. 608). This finding reinforces the assertion of Fosco and Grych (2007) that the issue of how culture affects children exposed to IPV warrants greater attention and further investigation.

The extent of emotional and physical abuse endured by mothers was a major factor in how children viewed the acceptability or even the necessity of physical violence in the family, as well as their beliefs in "the inherent superiority and privilege of men in the family" (Graham-Bermann \& Brescoll, 2000, p. 609). Younger age was an important factor in this effect. Younger children tended to feel that violence is a more acceptable 
way of resolving conflicts (Fosco \& Grych, 2007). At the same time, they are also more likely to blame themselves for conflicts between their parents, and they have more limited coping mechanisms compared to older children. The way children appraise situations of family violence has a marked impact on their psychosocial adjustment (Jouriles, Spiller, Stephens, McDonald, \& Swank, 2000).

Children who exhibited internalizing behavioral problems were more likely to view women as less powerful, but they did not necessarily attribute more power to men as some researchers have argued. Graham-Bermann and Brescoll (2000) concluded that gender stereotypes, attitudes toward the acceptability of violence, and the ages of children exposed to family violence should be focal points for cognitive interventions designed to help children reformulate detrimental attitudes and beliefs about gender and power. The results suggested that the need for intervention is greater for younger children and boys.

Family violence. A second perspective for examining IPV is the family violence perspective, which attributes the incidence of domestic violence to societal tolerance of violence as an acceptable means of resolving interpersonal conflict (Próspero, 2008). From this standpoint, any family member, male or female, may turn to violence in the face of family conflict. Proponents of the family-violence perspective have found that women and men are equally likely to turn to violence in conflict situations (i.e., gender symmetry of IPV).

Próspero (2008) and Graham-Kevan and Archer (2008) both invoked Johnson (1995), who argued that the feminist and family-violence perspectives represent two distinctive types of IPV and developed a framework for understanding domestic violence based on the motivation driving the perpetrator. In both types of IPV, control is the 
central theme. Johnson (1995) coined the term patriarchal terrorism to denote physical and psychological aggression carried out by men with the specific purpose of controlling the female partner. Forced economic dependence, isolation, intimidation, and threats are all control techniques used by men to control their partners. Victims of patriarchal terrorism are at high risk for physical injury and death, as evidenced by criminal-justice data (Catalano, 2007; National Center for Injury Prevention and Control [NCIPC], 2003). Statistics have shown that IPV causes close to 2 million injuries and almost 1,300 deaths among women each year (NCIPC, 2003). More than 555,000 of injuries resulting from IPV require medical attention and more than 145,000 require one or more nights in the hospital. Although there is no direct connection between the national data and patriarchal terrorism, Graham-Kevan and Archer (2008) pointed out that the accounts of IPV reported by women in battered women's shelters, along with the data from police and hospital records, convey an image of unrelenting male aggression against women consistent with patriarchal terrorism.

In Johnson's (1995) model, the family-violence perspective of IPV is labeled common couple violence. Partners who engage in this type of IPV are not driven by a need to control one another but rather to control situational conflicts that arise in family life. According to Johnson, the contrasting statistics reported by feminist researchers and family-violence researchers reflect different data sources. Evidence of the familyviolence perspective comes from general-population and college-student samples in contrast to the hospital, shelter, and criminal-justice records that support the feminist perspective. Recently, however, Johnson named patriarchal terrorism intimate terrorism 
in view of evidence showing that women could also exhibit a high degree of controlling behaviors combined with physical aggression (Graham-Kevan \& Archer, 2008).

\section{Intergenerational Transmission of Violence}

Similar to Graham-Kevan and Archer (2008), Próspero (2008) addressed Johnson's model of IPV in a study examining the role of control in the perpetration of IPV. In addition to Johnson's control typology, Próspero's study was guided by Bandura's (1977) social-learning theory, which is the framework for this dissertation study. Próspero noted that there is empirical evidence supporting a social-learning theory of interpersonal violence, specifically citing research showing that adolescents are more likely to perpetrate dating violence if they witnessed domestic violence at home, if they were witnesses to dating violence, or their peers were involved in antisocial behavior. Próspero grouped all three scenarios under the umbrella of social-learning theories. The study explored the effects of biological sex and gender-role orientation, along with controlling behaviors and IPV victimization, on attitudes toward three types of IPV perpetrations: physical, sexual, and psychological. The participants were 167 university students, roughly two thirds female (68\%) and 40\% Hispanic.

The instruments used for the study included the Revised Conflict Scale (CTS2) by Straus, Hamby, Boney-McCoy, and Sugarman (1996), the Bem Sex Role Inventory by Bem (1974), the revised Controlling Behaviors Scale devised by Graham-Kevan and Archer (2003) and used in their own research, and the revised Expagg of Archer and Haigh (1997), which assesses attitudes toward aggression (Próspero, 2008). Multiple regression analysis showed that IPV victimization, controlling behavior, violent attitudes, gender, and sex were significant factors in all three types of IPV perpetration, accounting 
for about $80 \%$ of the variance. IPV victimization and controlling behaviors emerged as the strongest predictors of IPV perpetration across all three analyses. With other factors controlled, masculinity and femininity were significant independent predictors of psychological IPV only, with higher masculinity linked with higher psychological IPV and higher femininity linked with lower psychological IPV.

Violent attitudes were only significantly linked with physical IPV only, with instrumental violent attitudes translating into higher physical IPV and expressive violent attitudes predicting lower physical IPV (Próspero, 2008). The findings for biological sex revealed that women were more likely to be perpetrators of psychological IPV, while men were more likely to perpetrate sexual IPV. In fact, college women have the highest risk of being victims of rape and other types of sexual assault than any other population group (Wasserman, 2004). Estimates for physical and psychological IPV victimization tend to show slightly higher rates of victimization among men or equivalent rates of victimization for men and women.

According to Próspero (2008), the findings highlight the complexity of understanding the perpetration of IPV. Jakupcak et al. (2002) and Moore and Stuart (2005) focused on men in their research on masculinity and violence. Próspero's analyses illustrated that the relationships between masculinity and violence also extends to women with a masculine profile. Participants with a masculine profile were more predisposed toward psychological IPV, while those with a feminine profile were less predisposed to perpetrate psychological IPV, irrespective of their biological sex. According to Próspero, this knowledge can be used to guide IPV intervention services that might operate on the stereotypical assumption that men are the perpetrators and women the victims of IPV. As 
a channel for future research, Próspero suggested examining the relationship between hypermasculinity and intimate terrorism with male and female perpetrators and across cultures. Negative machismo can be construed as a form of hypermasculinity.

IGTV and gender symmetry. Sugihara and Warner (2002) explored gender differences in dominance and aggressive behavior in partner relationships in a sample of 315 Mexican American men and women living in south Texas. The men and women had a mean age of 34 and 32 years, respectively; on the average, they had completed high school and some college and had income levels spanning a full socioeconomic spectrum. The CTS2 (Straus et al., 1996) was used in conjunction with the Dominance Scale, designed to assess domineering behavior among intimate partners.

Supporting the mutuality of aggression in partner relationships, the findings revealed almost equivalent levels of aggression by women and men, although women engaged in aggressive acts slightly less frequently (Sugihara \& Warner, 2002). Socioeconomic status was an important factor in the perpetration of violence. Lower income men and women were more likely to have physically assaulted a partner, and lower income men were more likely to report that they injured a partner. Sugihara and Warner found no evidence of traditional gender roles and stereotypes in this sample of Englishspeaking Mexican Americans who were at least second generation. In fact, both women and men displayed high levels of dominance, although the men had significantly higher decision-making power. Not surprisingly, Latina women were more eager to abandon traditional gender roles than men were to relinquish their traditional roles (Ruiz-Balsara, 2002; Snyder et al., 2010). 
Sugihara and Warner (2002) suggested that for some Mexican American men, aggressive behavior toward a partner may reflect an inability to adapt to changing gender roles. The assumption is supported by research on masculinity, gender-role stress, and violence perpetrated by men (Jakupcak et al., 2002; Moore \& Stuart, 2005). In particular, the violence displayed by lower income men, severe enough to injure a partner, may emanate from intense anger or rage in the face of discrimination and prejudice against Latino men in the United States (Sugihara \& Warner, 2002). Flores-Ortiz (1993) described a pattern of "cultural freezing" in which Latino men who have difficulty adapting to "Anglo" culture adopted exaggerated machismo and attempted to impose rigid gender-role expectations on their partners, including attempting to isolate their partner from Anglo culture. What Flores-Ortiz described is essentially a culture-specific form of intimate terrorism.

Sugihara and Warner (2002) concluded that "the stereotype of the 'macho' wife abuser is overly simplistic," and furthermore, women were far more likely to be aggressive toward their partners than to display marianismo (p. 332). Devaluation or lack of respect for the partner and possessiveness were both significant predictors of violence for men and women. It would be interesting to see the results if Sugihara and Warner had assessed intimate terrorism and common couple violence in their participants. Especially in conjunction with Próspero's (2008) study, Sugihara and Warner's (2002) findings dispel stereotypical assumptions about machismo and marianismo in IPV perpetration and victimization.

Acculturation. Harris et al. (2005) and Ramirez (2007) both explored the role of acculturation in IPV. Harris et al. focused specifically on female IPV victimization using 
data from the Mexican American Prevalence and Services Survey. A subset of 997 women living in a marital or cohabiting relationship were surveyed on the prevalence of physical and verbal aggression experienced in the last year by their current partners. The results showed that the greater degree the women adhered to traditional gender roles, the less likely they were to report incidents of IPV. Among domestic-violence survivors interviewed by Kasturirangan and Williams (2003), marianismo was a key factor in reluctance to report abuse. According to Harris et al. (2005), Mexican American women with very traditional gender-role attitudes may not recognize their partner's behavior as abuse. The researchers found it striking that gender-role beliefs had such a powerful role on reporting for both the Mexican-born and U.S.-born respondents, transcending sociodemographic characteristics and family dynamics that distinguished the two groups.

\section{Prevalence of Partner Violence Among Hispanic People}

Lown and Vega (2001) examined the lifetime prevalence of IPV, along with factors related to partner abuse, in a sample of 1,115 women of Mexican heritage living in Fresno, California, which has a large Hispanic population (38\%). A total of 127 women $(10.7 \%)$ reported being physically abused by a current partner.

Sociodemographic factors that increased the probability of abuse include being born in the United States, being young, residing in an urban area, being socially isolated, and having several children. Income status was not related to abuse, although Lown and Vega noted there was not much variance in income status among participants. Social support and church attendance emerged as protective factors against abuse and might have some interrelationship with support coming from other church members. 
Lown and Vega (2001) acknowledged that their findings of higher prevalence of abuse among women who were born in the United States and more acculturated may appear paradoxical, especially because women in that group enjoyed higher incomes and education, greater social support, and had fewer children. They speculated that some aspects of the traditional Mexican family might protect against domestic abuse. Another possible explanation is that the women might have been more acculturated and less inclined to accept traditional gender roles than their partners, which provokes an aggressive response in some Latino men (Sugihara \& Warner, 2002). Alternately, using the explanation of Harris et al. (2005), less acculturated Latina women may not always recognize their partner's aggressive behavior as abuse.

Hazen and Soriano (2005) investigated IPV in three groups of Latinas: women born in the United States, immigrant women, and migrant women. The sample consisted of 291 Latinas, primarily Mexican American. The findings disclosed high rates of abuse both in lifetime prevalence and of abuse experience within the last year. The overwhelming majority $(82.5 \%)$ experienced psychological abuse by a partner at some point, and close to three quarters (72.6\%) experienced psychological abuse during the past year. About one third experienced physical violence during their lives, and 18.5\% reported recent assaults. For sexual coercion, the figure was $20.9 \%$ for lifetime prevalence and $14.4 \%$ the past year.

Consistent with the findings of Lown and Vega (2001), Hazen and Soriano (2005) found that women who were more acculturated were more likely to have experienced IPV. The relationship between acculturation and IPV was due in part to higher prevalence of IPV among Latinas born in the United States. Nevertheless, high rates of partner 
violence are common in South and Central American countries (Flake \& Forste, 2006;

Lehrer et al., 2010; Rondon, 2003; WHO, 2005).

\section{Attitudes Toward Partner Violence: Honor Cultures}

Vandello and colleagues (Vandello \& Cohen, 2003; Vandello et al., 2009)

approached the issue of domestic violence from the perspective of cultural codes of honor that serve to justify violence against women who transgress traditional norms for "female chastity, purity, and modesty" (Vandello \& Cohen, 2003, p. 998). From this perspective, Vandello and Cohen conceptualized honor as a cultural syndrome that can promote maleto-female interpersonal violence. The researchers noted that virtually all cultures value honor, embodying qualities such as good moral character, integrity, virtuous behavior, and altruism. These qualities are equally admired in women and men. Honor can also be taken to mean status and reputation, usually in relation to male power and privilege. In cultures of honor, the second definition of honor is embedded in heavily stratified gender roles. Men preserve honor by exhibiting strength, toughness, and power, whereas cultural honor norms for women emphasize modesty and shame, and thus avoiding behaviors such as immodesty and adultery that would bring shame to the family.

Vandello and Cohen (2003) pointed out that the women in cultures of honor are not powerless in the sense that they are the bearers of the family honor. However, the "power" of women in such cultures comes largely from adhering to patriarchal and collectivist norms in which women's influence is primarily concentrated in the realm of interpersonal relationships. Machismo, marianismo, and familismo are obvious contributors to cultures of honor in Latin American countries. However, there are also more subtle and informal cultures of honor. Vandello and Cohen placed the U.S. South in 
this classification, noting that legal decisions in the South have, in some cases, legitimized and excused violence in response to adultery. Their research, which consisted of experimental studies in which university students were presented with scenarios, involved university students from Brazil and from northern and southern regions of the United States, including Hispanic students.

In their first study of honor cultures, Vandello and Cohen (2003) compared the responses of participants in Brazil and the United States. The researchers noted that there is a Brazilian expression, "Lavar a honra com sangue," meaning "wash the honor with blood," and such acts of violence (in some cases including murder) were accepted by Brazilian courts until quite recently (p. 999). In their subsequent research, the first study involved 273 students from college campuses in Sao Paulo and 350 students attending college in Illinois. The question driving the study was how a woman's infidelity would be seen to reflect on her partner, specifically whether he would lose his honor (i.e., be perceived as less manly and trustworthy) and whether or not violence would be justified as a response. The first scenario depicted a couple in which the wife was either faithful or was having an affair of which the neighbors were aware. The second scenario portrayed a couple that had been married for 7 years when the husband found out his wife was having an affair and responded by either yelling at her to end the affair immediately or with physical violence. Two other versions depicted the husband either doing nothing or saying he wanted a divorce.

Reflecting the culture of honor tradition, the Brazilian students viewed the man as being less trustworthy and less manly if his wife was unfaithful than if she was not unfaithful (Vandello \& Cohen, 2003). For the U.S. students, the wife's fidelity or 
infidelity had no effect on their perceptions of his trustworthiness and good character and a much more limited effect on his perceived masculinity (i.e., the effect on masculinity was twice as strong for the Brazilian sample). Conversely, the U.S. students viewed the woman's infidelity as more compromising to her trustworthiness and good character than did the Brazilian students. With regard to the second scenario, the husband's response to infidelity, the Brazilians viewed the man who hit his wife as slightly more manly than the man who yelled at her. Among the U.S. respondents, the man who hit his wife was perceived as less trustworthy and less manly. Additionally, the U.S. students felt that the man who responded with physical violence loved his wife less than the one who yelled at her, whereas the Brazilian students made no distinction between the two. Neither group of students approved of the man hitting his wife, but the Brazilians tended to be more willing to excuse the violent act.

Vandello and Cohen (2003) acknowledged that none of the effect sizes were large, reflecting the numerous individual differences of respondents in each cultural group. No effects for the participants' gender emerged in the analysis. The second study involved 112 students from the University of Illinois at Urbana-Champaign, classified according to ethnicity and region of origin. The sample consisted of 33 Hispanic students, 41 White students from the northern United States, and 38 White students from the southern United States. After filling out lengthy demographic questionnaires, participants arrived individually to find a sign saying the experimenter was late; after being seated they were confronted with a staged scenario by a male and female confederate (i.e., enacting the roles of study participants) who staged a loud, heated argument involving the woman's intention to visit a former boyfriend and the man's attempt to stop her, which 
escalated into the man's forceful shoving the woman into a wall and leaving her with the words, "I'll see you at home," spoken in an intimidating manner. The scenario was manipulated so that for half the participants, the woman accepted the violence (i.e., "contrite") and for the other half, she was angry and ready to leave him (i.e., "notolerance").

After the scene, the experimenter arrived, telling the participants that they were in an experiment about impressing formation in which they would be chatting with another participant: the female confederate who assumed a personality that was the antithesis of her response in the previous role play (i.e., assertive or self-blaming). According to Vandello and Cohen (2003), the change in response was meant to portray conflicting emotions over the relationship. The two confederates carefully observed the responses of the participants who completed a questionnaire on the justifiable nature of various conflict situations. Ten participants were dropped from the analysis because they expressed some skepticism over the credibility of the scenarios, perhaps a hazard of that type of experiment. However, the researchers noted that the presence or absence of their responses did not change the results.

The results of the study supported the idea that differences would emerge in the reactions of the Hispanic and White southern participants and the reactions of the students from the north (Vandello \& Cohen, 2003). Although the participants from the two honor cultures expressed a more favorable view of the woman who displayed contrition and loyalty after the physical conflict with her "fiancé," the northern participants favored the woman who was independent and refused to tolerate his abusive behavior. Furthermore, the northerners perceived the woman who stayed with her partner 
as weak, whereas the southern and Hispanic students viewed them as equally strong in both cases. To the southerners and Hispanic students, the woman displayed more warmth and goodness by staying. The female confederate affirmed that the southern and Hispanic participants were more inclined to convey tolerance for the male's aggression and suggested she remain in the relationship.

Vandello and Cohen (2003) emphasized that the group differences did not capture the full range of individual responses that surfaced in each cultural group. This effect underscores the danger of imposing cultural stereotypes to explain the behaviors of members of any ethnic, cultural, or gender group (Ahrens et al., 2010). Nevertheless, there was evidence of the role of cultural scripts in the responses of the students to the written and enacted scenarios. Vandello and Cohen (2003) viewed understanding of how cultural scripts defined how women and men are supported to act in social relationships as essential to fully understanding the phenomenon of domestic violence.

In subsequent research into domestic violence and honor cultures, Vandello et al. (2009) focused on marianismo, conceptualized as loyalty and self-sacrifice in the face of an abusive relationship. The first study involved White southern, White northern, and Hispanic students attending the University of Illinois, as in the earlier research exploring different reactions to a woman's response to relationship violence (Vandello \& Cohen, 2003). The sample of 163 University of Illinois students were shown a brief video of a woman describing an incident where she told her fiancé that she was driven home by a male coworker and he responded with jealousy, escalating into an argument that culminated in his hitting her in the face (Vandello et al., 2009). The woman (whose ethnicity was ambiguous) was described as Mexican American to the Hispanic 
participants and White to the White participants. There were three different versions of the scenario: in Version 1, the woman left the relationship; in Version 2, the woman said she was angry but supported him and "tried to 'love away' his wild edge and he never hit her again"; Version 3 was the same as Version 2, but the woman admitted he hit her again "a couple of times" (p. 86).

The participants expressed a far more favorable opinion of the woman who left the abusive partner than the woman who stayed, and they appraised the conflictresolution strategy of leaving much more positively (Vandello et al., 2009). When analyzed according to culture, the participants from honor cultures (i.e., Hispanic and southern White people) were somewhat more positive toward the woman who stayed, appraising her as warmer as and smarter than did the White northern participants. As in the earlier study, the effect sizes reflected a range of individual differences in each group, and Vandello et al. (2009) emphasized that the general tendency favored the woman who left the abusive partner.

According to Vandello et al. (2009), the "complementary expectations for female loyalty and male defense of honor in jealousy-threatening situations" might help to account for the relatively high rates of domestic violence in Latin American cultures and in the southern United States, and also provide insight into why abused Latinas are more likely to remain in violent relationships compared to their Anglo American counterparts (p. 99). Although machismo and marianismo are almost invariably mentioned in research on IPV among Latinas, few researchers seem to have expanded into the related concept of culture of honor. 


\section{Spirituality and Religion}

Religious leaders' beliefs. The literature has suggested that for many women, formal religious involvement is more likely to inhibit their ending an abusive relationship than facilitating it (Lee, 2007; Roberts, 2006). Unlike mental health professionals who view a woman's abuse history as a mechanism for understanding her current situation, some religious clergy proposed that, "victims desired abuse due to childhood abuse experiences leading to low self-esteem" (emphasis in original, Levitt \& Ware, 2006, p. 220). Consequently, "because leaders believed that victims were desirous of abuse, they expressed exasperation and bafflement about interacting with victims of abuse" (p. 220).

The above statements were generated by Levitt and Ware (2006). They emphasized that the Jewish, Christian, and Islamic clergy expressed a wide range of perspectives, and many views were inconsistent with professional knowledge of IPV. Most endorsed ideals of love, trust, respect, and communication as essential elements of marriage, but these are out of touch with the victims' actual lives. The majority endorsed separation as an issue of safety. However, those with fundamentalist ideals in particular were resistant to divorce. The authors noted that this has the ability to compromise the safety of women who turn to clergy for guidance.

\section{Chapter Summary}

Criminal justice data mask the insidious presence of psychological abuse in perpetuating physical violence. Psychological abuse typically occurs in conjunction with physical violence (Fraser, McNutt, Clark, Williams-Muhammed, \& Lee, 2002; Wrangle, Fisher, \& Paranjape, 2008). Psychological abuse fosters feelings of worthlessness, powerlessness, shame, fear, and isolation, exacerbating the damaging effects of physical 
violence (Smith \& Randall, 2007; WHO, 2005). A focus of psychological abuse is maintaining control, and in the most direct manifestations, abusive partners prevent women from working or accessing healthcare services (McCloskey et al., 2007; Nam \& Tolman, 2002).

The IGTV is a popular framework for examining IPV. The strongest support comes from research with abusers, the greater the exposure to domestic violence in childhood, the higher levels of violence they display within and outside of the relationship (Murrell, Christoff, \& Henning, 2007; Torres \& Han, 2003). Although there has been less direct evidence for the intergenerational theory in victims of IPV, childhood physical and sexual abuse are extremely common in the histories of women who experience IPV (Allen, 2001; Bassuk, Dawson, \& Huntington, 2006; Coker et al., 2000; DeJonghe et al., 2008; Glass et al., 2008; Roberts, 2006; Sansone, Chu, \& Wiederman, 2007; Schewe, Riger, Howard, Staggs, \& Mason, 2006; Tjaden \& Thoennes, 2000; Weis, Fine, Proweller, Bertram, \& Marusza, 1998; WHO, 2005). Additionally, adolescents exposed to family violence may exhibit aggressive behavior, linked with the presence of PTSD (Moretti et al., 2006).

There is relatively minimal research on the attitudes of Latinas or Latinos, particularly college students, toward IPV, or on how it relates to IGTV. Much of the existing research on IPV in Hispanic populations is driven by the assumption that genderrole stratification embodied by the concepts of machismo and marianismo is a powerful factor in partner violence. There is some evidence supporting this view. However, there is also compelling evidence that the power of culturally prescribed gender roles is diminishing and Latin men and women do not adhere to stereotypical gender roles. 
Mexican and Mexican American men and women are equally likely to be perpetrators and victims of IPV, consistent with their White Anglo counterparts (Straus \& Ramirez, 2007; Sugihara \& Warner, 2002). Women as well as men with a masculine gender-role orientation may perpetrate partner violence as a means of control (Próspero, 2008).

Studies have generally found that Latinas who are more acculturated are more likely to be victims of IPV (Cuevas et al., 2010; Hazen \& Soriano, 2005; Lown \& Vega, 2001). These findings also challenge the notion that IPV is linked with traditional gender roles. However, there is evidence that women are more likely to embrace the freedom and independence they gain from discarding traditional gender roles than men are to relinquish their traditional power. Differences in levels of acculturation by partners in a relationship can be a factor in IPV. It is also noteworthy that many researchers who investigate IPV victimization in women do not assess whether the women are also perpetrators of IPV. There is abundant evidence of gender symmetry in IPV (GrahamKevan \& Archer, 2008; Próspero, 2008; Straus \& Ramirez, 2007).

There is also evidence that Latin cultural values regarding gender, relationships, and sexuality influence college students' attitudes toward IPV. Honor culture rather than gender roles per se is an important and intriguing influence that offers a useful framework for examining attitudes toward IPV in different cultural groups (Vandello \& Cohen, 2003; Vandello et al., 2009). Thus, it is important to note that the prevalence of IPV on college campuses makes it an important issue that merits greater research attention. With the increasing diversity of the U.S. college population, cross-cultural research would serve to illuminate differences and similarities across and within groups for the purpose of designing campus primary prevention and intervention campaigns. 


\section{CHAPTER III}

\section{METHODOLOGY}

\section{Design}

This research used a mixed-methods convergent design, which involved administering quantitative measures related to IPV, cultural factors, and childhood risk factors triangulated with qualitative data collected through administration of a paper-andpencil open-ended questionnaire (see Figure 2). HCSs were recruited by the principal investigator (PI) and undergraduate research assistants (RA). Data on gender, religiosity, cultural gender roles, level of victimization, level of perpetration, adult recall of parental IPV, acculturation, and perceptions of IPV were collected.

\section{Setting}

Recruitment took place in two south Florida universities, one located in MiamiDade County and one located in Palm Beach County. The PI was familiar with these two university settings and was confident that the desired sample of participants needed in the study could be obtained. The locations of data collection were on-campus sites such as classrooms or meeting room areas in a school.

Figure 2 is the Procedural diagram for intimate partner violence: Perceptions of Hispanic college students, convergent parallel mixed-method design adapted from Creswell and Plano Clark (2011). 


\section{Procedures:}

$\sim$ Recruited 120

participants who are

HCS, 18-25 years old,

speak English; either

with a partner or

currently dating or

having been in a dating

relationship.

\section{SURVEY MEASURES:}

$\sim$ Demographics, ERS

SASH, CTS2 CTS2-CA, and $\mathrm{FPB}$; perceptions of

IPV using two close-

ended questions

\section{Procedures:}

$\sim$ Descriptive statistics

Group comparisons

$\sim$ correlation techniques

$\&$ multiple regression

analysis

\section{Products:}

$\sim$ Numerical item scores
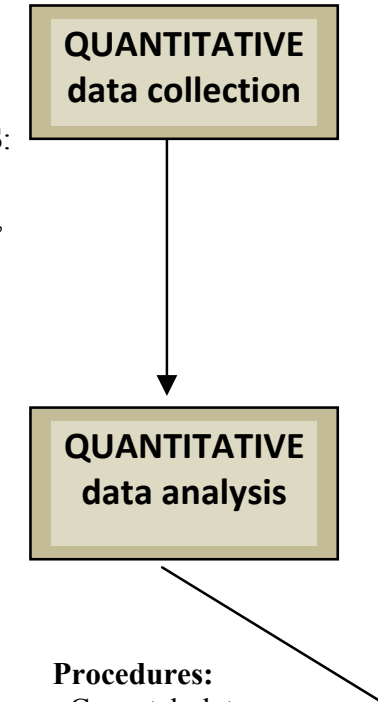

$\sim$ Cross tabulate qualitatively derived groups with quantitative variables

Procedures:

$\sim$ Consider how merged results contextually define IPV

\section{Products:}

IPV, acculturation and

religiosity among HCSs.

$\sim$ Mean, SD, Correlation

\section{$\sim$ Multiple Regression}

\section{Procedures:}

$\sim$ Randomly selected

sub-sample of 20

participants

\section{QUALITATIVE \\ data collection}

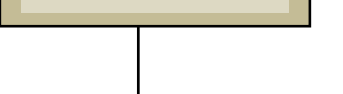

levels of cultural gender

roles, adult recall of parental

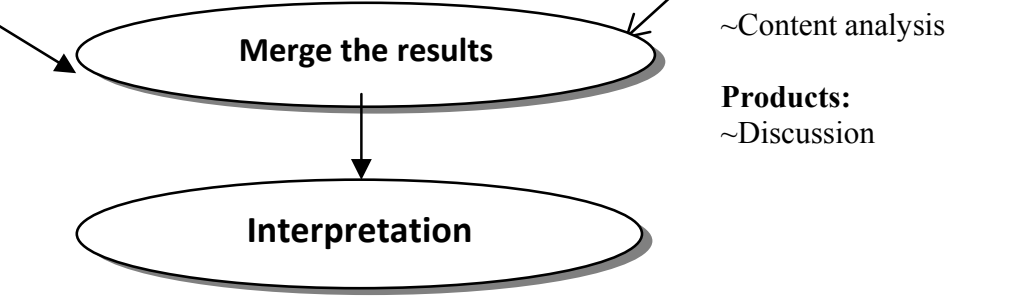

\section{Products:}

Answers obtained from 4 open-ended

questionnaires: (a) What does intimate partner violence means to you? (b) What do you think intimate partner violence means to your parents? (c) Do you believe that your definition of intimate partner violence is similar to that of your parents? (d) Tell me about what kind of violence there are.

\section{Products:}

$\sim$ Content analysis $\sim$ Typology of HCS's perceptions of IPV

Figure 2. Procedural diagram for intimate partner violence: Perceptions of Hispanic college students, convergent, parallel, mixed-method design. FPB $=\mathrm{Cultural}$ Gender Role; CTS2-CA = Adult Recall of Parental IPV; SASH = Acculturation; ERS =Religiosity; CTS2 = Past \& Current Experiences of IPV; HCS = Hispanic college student; IPV $=$ Intimate Partner Violence 


\section{Sample}

A sample of 120 students consisted of any Hispanic or Latino(a) male or female college students who self-identified as being of Mexican, Puerto Rican, Cuban, Salvadoran, Dominican, Guatemalan, Colombian, Honduran, Ecuadorian, or Peruvian ethnicity. Participants needed to able to read and understand spoken and written English, since all data-collection forms were in English and completed by participant self-report. Inclusion criteria were (a) college students who self-identified as Hispanic/Latino/a; (b) male and female; (c) between the ages of 18 and 25 years; (d) currently in a relationship with one partner (i.e., married or unmarried) or in a relationship in the past year (i.e., married or unmarried) at the time of recruitment; and (e) currently residing (or in the past resided) with two biological parents or a biological parent and stepparent during childhood. Students who complied with the above criteria were recruited for participation in the study. Participants were required to be English-speaking and able to properly communicate with the researcher. Exclusion criteria included any self-identified Hispanic/Latino/a college student who verbalized or indicated inability to understand the research process. Also excluded were non-Hispanic/Latino college students and any individuals beyond the desired age parameters (i.e., 18 to 25 years old), since the project required the study of HCSs referred to as emerging adults.

Sampling and recruitment. A total of 120 HCS participants were targeted to participate in the study. Twenty participants as a subsample were randomly selected to explore HCS perceptions of IPV. Personal face-to-face and telephone contacts, flyers, and e-mail were used as recruitment tools to find participants for the study. In this study, five instruments including a student survey were used to collect data and measure 
intergenerational transmission of abuse. These are: the Familial Patriarchal Belief (FPB) scale, the Conflict Tactics - Adult Recall (CTS2-CA), the Short Acculturation Scale for Hispanics (SASH), the Extent of Religiosity (ERS), and the Revised Conflict Tactics Scale (CTS2) (see Appendix B). In addition, a student survey and a set of researcherdeveloped open-ended questions were created to discern IPV perceptions and demographic attributes of the participants in this study.

Additional questions were included at the beginning of the survey to obtain demographic information such as age, gender, cultural background, socio-economic status, religion, partner/relationship status. Two questions were created to obtain information about participants' perception of victimization and perpetration. And four open-ended questions were added at the end of the survey to obtain additional information about participants' experiences and views about IPV.

Sources of materials. The snowball recruitment method was used by the PI/RA to recruit potential study participants in the two target universities located in southeast Florida. The snowball recruitment method is a technique for finding research subjects. One subject gives the researcher the name of another subject, who in turn provides the name of a third, and so on (Vogt, 1999). This strategy can be viewed as a response to overcoming the problems associated with sampling. This process is based on the assumption that a 'bond' or 'link' exists between the initial sample and others in the same target population, allowing a series of referrals to be made within a circle of acquaintance (Berg, 1988). Upon identification of potential participants, HCSs were asked about their interest to participate in the "Hispanic College Students' Relationship" study. Once eligibility was determined, the PI/RA informed prospective participants that this research 
study was being conducted by a $\mathrm{PhD}$ in Nursing student at Florida International University. Consequently, study objectives were discussed, informed consent obtained, and the study survey was administered. Data were collected from HCSs using paper-andpencil questionnaires for the instruments noted in this dissertation.

Quantitative analysis. According to LoBiondo-Wood and Haber (2006), nonexperimental descriptive research approaches help researchers measure the intensity of the correlations between the variables by "quantifying the strength of the relationship between the variables or in testing a hypothesis about a specific relationship" (p. 242). The Statistical Package for the Social Sciences ${ }^{\circledR}$ (SPSS) 17.0 was used for data analyses for the cultural gender roles (FPB), adult-recall of parental IPV (CTS2-CA), acculturation (SASH), religiosity (ERS), and experiences of IPV (CTS2) among HCSs (see Table 2). The CTS2 and CTS2-CA instruments were the only measures that required "license use agreement” for above-mentioned study instruments (see Appendix A). The FPB scale, SASH and ERS are public domain instruments.

\section{Research Hypotheses}

Five exploratory research hypotheses were tested in this study:

$\mathrm{H}_{1}$ : Perceptions of IPV will be directly related to gender, cultural gender roles, adult recall of parental IPV, acculturation, religiosity, level of victimization, and level of perpetration.

$\mathrm{H}_{2}$ : There is a relationship between gender, cultural gender roles, adult recall of parental IPV, acculturation, religiosity, and level of victimization.

$\mathrm{H}_{3}$ : There is a relationship between gender, cultural gender roles, adult recall of parental IPV, acculturation, religiosity, and level of perpetration. 
$\mathrm{H}_{4}$ : There are relative contributions of cultural gender roles, adult recall of parental IPV, acculturation, and religiosity to level of victimization.

$\mathrm{H}_{5}$ : There are relative contributions of cultural gender roles, adult recall of parental IPV, acculturation, and religiosity to level of perpetration.

\section{Power Analysis}

To determine an adequate and appropriate sample size for this study for the number of variables and proposed statistical-analysis techniques, a priori power analyses were conducted using the G*POWER 3.0 to determine the sample size needed to conduct the study. G*POWER is a power-analysis program "designed as a standalone application to handle several types of statistical tests commonly used in social and behavioral research" (Faul, Erdfelder, Lang, \& Buchner, 2007, p. 175). The software package has received favorable ratings for accuracy and precision and is available, at no charge, on the Internet (Goldstein, 1989). Estimated sample size for this study using G*POWER were comparable with those listed in Cohen's $(1988$, p. 55) sample size tables. The study included five predictor variables and two criterion variables. Power analyses were conducted for bivariate correlational and multiple-regression analyses.

In view of the relative seriousness of possibly committing a Type I or Type II errors in this survey study, alpha ( $\alpha$ ) was set at the conventional level of .05 , and beta ( $\beta$ ) was set at the conventional level of .20, or four times alpha. As a result, the desired power was calculated as $1-\beta=.80$.

Research Question 1 was addressed using descriptive and content analysis to evaluate whether HCSs perceived themselves as victims/survivors of IPV or perpetrators of IPV while in a relationship. The first part of Research Question 1 consisted of two 
inquiries that were answerable by yes or no. These questions were: (a) "Do/did you consider yourself a victim/survivor of a partner or dating violence?" and (b) "Do/did you consider yourself a perpetrator of violence while in a relationship?" Content analysis was utilized for the second part of Research Question 1 for the four open-ended questions.

Prior to running statistical analyses, tests of normality of major variables were performed (Table 1). Descriptive statistics were provided as means and standard deviations for continuous variables, and numbers of responses and proportions for binary and categorical variables. All continuous variables were tested for normality using Kolmogorov-Smirnov and Shapiro-Wilk test and both tests provided similar significant results. Results of the Shapiro-Wilk test is shown in Table 1 since it is a powerful test for sample sizes between 50 to 2000 (Royston, 1992). Descriptive analysis and correlational techniques (i.e., chi-square, Pearson's $R$ ) were used to test for associations between independent variables cultural gender roles, adult recall of parental IPV, acculturation, and religiosity among HCSs; and dependent variables level of victimization and level of perpetration were used to answer Research Question 2. Although exploring gender differences goes beyond the study of this dissertation, it is included in the study as it added layers of understanding in exploring whether attitudes towards gender role differ based on gender. The Mann-Whitney $U$ test was used to draw different conclusions about gender differences.

To answer Research Questions 3 and 4, correlational techniques (i.e., chi-square, Pearson's $R$ ) were used to determine the level of relationship that exists between variables. Power analysis for Research Questions 3 and 4, using the exact correlation, 
bivariate two-tailed model, yielded a total required sample size of 84 participants needed for an alpha set at .05 and a power of .80 .

Prior to running the relevant linear regression analysis, dependent variables, level of victimization and level of perpetration; and the independent variables, cultural gender role, religiosity, and adult recall were transformed using the square-root transformation. All continuous variables were transformed to approximate the normal distribution in order to satisfy the assumption of normality to perform regression analysis. Although, $\log 10$ and natural- $\log (\ln )$ are the most commonly used variable transformation, neither of these methods was suitable for this dataset due to the inclusion of 0 values. Thus, the square root transformation was utilized (Marcus, Lindahl \& Neena, 2001).

Regression analyses (ENTER method) were then used on the square-root transformed variables to identify significant relationships between dependent and independent variables. This was followed by a regression analysis (ENTER method) carried out in SPSS. Multiple regressions were used for Research Questions 5 and 6 to predict the relative contributions of gender, cultural gender roles, adult recall of parental IPV, acculturation, and religiosity scores on the level of victimization and level of perpetration among HCSs. For Research Questions 5 and 6, power calculations indicated that with a medium effect size of .25 , with the alpha set at .05 , and a power of .80 ; a sample of 92 was needed to achieve significance. Missing data was dummy coded at the time of data entry and listwise deletion was utilized prior multiple regressions.

Given the estimated sample size and accommodating for missing data which may decrease the power, and the possibility that some returned questionnaires would be unusable for analyses, the desired sample size for this study was 120 . There was no doubt 
that the desired sample size would be obtained because data collection was be open to all HCSs in two south Florida universities who are 18 to 25 years old and wanted to participate in the study.

Table 1

Tests of Normality for Cultural Gender Roles, Adult Recall of Parental IPV, Acculturation, Religiosity, Past and Current Experiences of IPV, Level of Victimization and Level of Perpetration

\begin{tabular}{lccc}
\hline \multicolumn{1}{c}{ Scales } & $\begin{array}{c}\text { Shapiro-Wilk } \\
\text { Statistic }\end{array}$ & Df & p-value \\
\hline Cultural Gender Roles (FPB) & 0.881 & 120 & 0.000 \\
$\begin{array}{l}\text { Adult Recall of Parental IPV } \\
\text { (CTS2-CA) }\end{array}$ & 0.915 & 89 & 0.000 \\
Acculturation (SASH) & 0.984 & 120 & $0.157^{*}$ \\
Religiosity (ERS) & 0.949 & 120 & 0.000 \\
$\begin{array}{l}\text { Past and Current Experiences of IPV } \\
\text { (CTS2) }\end{array}$ & 0.670 & 86 & 0.000 \\
Level of Victimization & 0.511 & 103 & 0.000 \\
Level of Perpetration & 0.469 & 102 & 0.000 \\
\hline
\end{tabular}

$*>.05=$ normally distributed.

\section{Qualitative Analysis}

At the end of data collection, data and content analysis were performed. Content analysis is "any technique for making inferences by systematically and objectively identifying special characteristics of messages" (Holsti, 1968, p. 608). The strategy applied by the PI was to assess answers on written documents (survey) from the randomly selected sample of participants. Sampling of content analysis was applied on 
participants' answers based on the four open-ended questions; and occurred at any or on all of the following levels: words, phrases, sentences, and paragraphs that were relevant to the context of IPV variables being explored. Similar concepts were gathered or clustered together into conceptual clusters or ideas that constitute variables of interest. The PI adapted the method of cognitive processes (Morse, J., 1994) that is inherent in content analysis. This involves comprehending, synthesizing, theorizing, and recontextualizing (p.25). Various concepts were reported and discussed accordingly with illustrated examples of quotations. To explore HCSs' perceptions of IPV, the PI used a paper-and-pencil open-ended questionnaire and a "yes" or " $n$ " answer these questions as formulated by the PI: (a) Do/did you consider yourself a victim/survivor of a partner or dating violence? and (b) "Do/did you consider yourself a perpetrator of violence while in a relationship?"

\section{Quantitative Method}

The quantitative method originated in the philosophical domain of "logical positivism, which operates on strict rules of logic, truth, laws, and predictions. ... To find the truth, the researcher must be completely objective, meaning that values, feelings, and personal perceptions cannot enter into the measurement of reality (Burns \& Groves, 2005, p. 23). For nurse researchers, the foundation of qualitative studies is focused mainly on the philosophy of postpositivism (Clark, 1998), and "truth can be discovered only imperfectly and in probabilistic senses, in contrast to the positivist ideal of establishing cause and effect explanations of immutable facts" (Ford-Gilboe, Campbell, \& Berman, 1995, p. 16). 
A nonexperimental descriptive research approach was employed for the quantitative portion of this study to help "describe and explain the nature of [an] existing relationship, without necessarily clarifying the underlying causal factors in the relationship" (Fain, 2004, p. 210) of the variables of interest in this study. Demographic variables were measured by using a simple demographic questionnaire that contained questions pertaining to HCS identified race/ethnicity, country of origin, religious background, and socioeconomic status.

The Familial Patriarchal Belief (FPB) scale was used to assess the levels of cultural gender roles. This is a five-item, 5-point Likert-type scale. A Cronbach's alpha estimate was .79 for the English version (Smith, M. 1990). The Conflict Tactic Scale Adult Recall (CTS2-CA) was used to assess HCS past or present parental exposure to violence between parents and caregivers (Straus et al., 1996). The Short Acculturation Scale for Hispanics (SASH) is a 12-item scale use to identify low and high acculturation. It assesses language use, preferences regarding media and social relationships, and participants' generation, length of residence in the United States, age of arrival, and ethnic self-identification (Marín, Sabogal, VanOss Marín, Otero-Sabogal \& Pérez-Stable, 1987). The Extent of Religiosity (ERS) measured HCS religiosity level. This tool is a three-item scale constructed specifically to measure the level of religiosity among Arab men (alpha coefficient $=.85)$ in Israel (Haj-Yahia, 2003). To date, this measure has not been used with an HCS sample. This scale was included in the demographic portion of the survey. Lastly, the CTS2 is a 74-item self-report instrument was used to measure the level of HCS level of victimization and perpetration. This tool is composed of five scales used to assess the following dimensions: negotiation, psychological aggression, physical 
assault, sexual coercion, and injury between partners (Straus, Hamby, Boney-McCoy, \& Sugarman, 1995; Straus et al., 1996). For each scale and subscale, lifetime and past experiences of abuse can be obtained (see Table 2).

\section{Qualitative Method}

A qualitative approach was used to identify the dynamics and dimensions of understanding IPV through HCS perceptions and experiences. "The idea that multiple realities exist and create meaning for the individuals studied is a fundamental belief of qualitative researchers (Speziale \& Carpenter, 2007, p. 21).

The qualitative data collection method involved open-ended paper-and-pencil questions. These questions provided the means of generating data from HCSs in order to discover their perceptions and experiences of IPV and explore any congruencies of meanings based on HCSs' gender, cultural gender roles, adult recall of parental IPV, acculturation, and religiosity.

Of a total of 120 HCSs surveyed, 20 were randomly selected from the participants to further explore HCSs' perceptions of IPV by contextually analyzing their answers generated from the four open-ended questions. The participant selection consisted of a convenience sample of HCSs in two university settings, one located in Miami Dade County and the other in Palm Beach County. Although this was a convenience selection, south Florida is a socioeconomically and ethnically diverse community whose populations reflect the demographic characteristics of the study sample. Participants were male and female, 18 to 25 years of age, and attending a public or private university. Participation was voluntary, and confidentiality was always ensured. 


\section{Instruments}

The perception of quantitative researchers is that "all human behavior is objective, purposeful, and measurable ... [and] needs only to find or develop the right instrument or tool to measure the behavior" (Burns \& Groves, 2005, p. 23). The selection of diagnostic instruments necessitated diligent inspection for authenticity since it influences the findings of research studies (LoBiondo-Wood \& Haber, 2006). In this study, five instruments were used to collect data and measure intergenerational transmission of abuse: the FPB scale, the CTS2-CA, the SASH, the ERS, and the CTS2 (see Appendix B). In addition, a student survey and a set of researcher-developed open-ended questions to discern IPV perceptions and demographic attributes of the participants in this study.

Student survey. A descriptive survey that included HCS demographic data and a semi structured questionnaire was used to describe the sample volunteer participants in this study. The questionnaire included four sets of guided questions and open-ended questions to stimulate the exploration of HCS perceptions of IPV. Also included was a set of questions that determines the age, gender, cultural background, religion, and relationship status of the volunteer respondents. According to Speziale and Carpenter (2007, p. 21), "The idea that multiple realities exist and create meaning for the individuals studied is a fundamental belief of qualitative researchers." Thus, this allowed for the multicultural/multiethnic perspectives of the diverse population that would make up the HCS participants.

Overview of study measures. The survey has been developed to study HCS perceptions and experiences with various types of IPV and examine associations among levels of cultural gender roles, adult recall of parental IPV, acculturation, and religiosity 
among HCSs. Table 2 provides the measures used to operationalize HCS perceptions of IPV, cultural influences of IPV (cultural gender roles, acculturation, and religiosity), and abuse status (adult recall of parental IPV, level of victimization, and level of perpetration).

\section{Procedure: Protection of Human Subjects}

Potential risks. Potential psychological risk, sense of shame, embarrassment, and stigma may be brought about because of the sensitive nature of the study and concepts included in the questionnaires. Interventions included the provision of information about battering, shelter telephone numbers, and varying degrees of emotional support and therapeutic communication. A list of resources was also provided in the event that psychological assistance was needed.

Legal issues which may arise was anticipated as PI or RA have the duty to warn IPV victims if identified during the course of the survey. Confidentiality, which was maintained throughout the study, may be breached if the PI or RA determines, from information obtained during the survey of the batterer that the intended victim is in danger. Social service agencies or battered women's shelters generally have legal information as well as links with legal services. This information was given to all participants during the survey. Breach in confidentiality was also prevented through the anonymity of the survey packets and storage of data in secure and locked premises.

Adequacy of protection against risks. The safety of the participants was considered in all stages of the research process from the initial contact, interview, data collection, and follow-up. The participants involved in this study are HCSs, who may or may not consider themselves as "abused" by an intimate partner. Potential risks among 
men and women who may have already suffered abuse based on clinical and criminal justice's definitions of IPV, increases the need for the researcher to provide interventions, especially if the battered man or woman is returning to a dangerous home situation. It is sensible to accept these potential problems with the data involved and the PI and RAs were prepared to intervene. These interventions, if the situation arise, include providing information about battering, shelter phone numbers, legal information, and varying degrees of emotional support and therapeutic communication. Potential psychological risk was anticipated; thus, interventions included providing information about battering, shelter telephone numbers, legal information, and varying degrees of emotional support and therapeutic communication. Once willingness to participate was identified, the PI/RA met at an agreed upon location chosen by the participant (e.g., college/university campus or community center) to obtain informed consent. The consent form was approved by the participating universities in the study. Research assistants received training in all the procedures to ensure confidentiality of all the data collected.

Safety issues are of great concern for IPV at-risk participants, especially if they are solicited by public advertising such as flyers and electronic mail. On the advertisement, the PI/RA's TracFone ${ }^{\circledR}$ mobile or telephone numbers, and e-mail addresses were included. Participants were self-identified Hispanic/Latino(a) college students living in southeastern Florida. Information for participants was delivered on an informed consent form. Once potential participants were identified by the PI/RA, they were contacted via telephone or however each participant preferred and were provided with explanation of the study and an overview of the study goals. 
During data collection, each participant completed the demographic/student survey forms and survey instruments. These include the FPB (Smith, M. 1990), the CTS2-CA (Straus et al., 1996), the SASH (Marin, et al., 1987), the ERS (Haj-Yahia, 2003), and the CTS2 (Straus, Hamby, and Warren, 2003). Qualitative data was also collected within the same session open-ended questions were part of, and were included within the survey packet.

The study questionnaires were completed by individual participants. Each datacollection visit or individual session entailed approximately 60 minutes (i.e., 1 hour) to complete. Each participant who signed the consent and began the study received a $\$ 5$ Starbucks ${ }^{\circledR}$ gift card once they stated that they completed the study questionnaire. 
Table 2

Description of Study Measures

\begin{tabular}{|c|c|c|c|c|}
\hline Scale assessment & Construct & Characteristics* & Psychometrics & Developer \\
\hline $\begin{array}{l}\text { Perceptions of IPV } \\
\text { questionnaire }\end{array}$ & $\begin{array}{l}\text { To assess HCS perceptions } \\
\text { of IPV. }\end{array}$ & $\begin{array}{l}4 \text { open-ended questions were } \\
\text { created for contextual analysis. } \\
\text { Examples of the open-ended } \\
\text { questions are: "What does intimate } \\
\text { partner violence mean to you?" and } \\
\text { "What do you think intimate partner } \\
\text { violence meant to your parents?" }\end{array}$ & Not applicable & \multirow{5}{*}{$\begin{array}{l}\text { Straus et al. } \\
(1996) \text {; } \\
\text { Straus, Hamby, } \\
\text { and Warren } \\
(2003)\end{array}$} \\
\hline \multirow{4}{*}{$\begin{array}{l}\text { The Revised } \\
\text { Conflict Tactic Scale } \\
\text { (CTS2) }\end{array}$} & \multirow{3}{*}{$\begin{array}{l}\text { To assess HCS physical } \\
\text { victimization and } \\
\text { perpetration. } \\
\text { This self-report measure } \\
\text { includes psychological and } \\
\text { physical attacks on a } \\
\text { partner and the use of } \\
\text { negotiations in a marital, } \\
\text { cohabiting, or dating } \\
\text { relationship. }\end{array}$} & $\begin{array}{l}78 \text {-item scale that assesses both } \\
\text { victimization and perpetration. }\end{array}$ & $\begin{array}{l}\text { Internal consistency: (men \& women } \\
\text { combined) Physical Assault }=.86\end{array}$ & \\
\hline & & $\begin{array}{l}\text { An 8-point Likert scale ranging } \\
\text { from } 0 \text { (this has never happened } \\
\text { before) to } 6 \text { (more than 20x in the } \\
\text { past year) and } 7 \text { (not in the past } \\
\text { year, but it did happened before) } \\
\text { response. }\end{array}$ & $\begin{array}{l}\text { Internal consistency: Physical = .90 } \\
\text { (Mechanic, Weaver, \& Resick, } \\
\text { 2000); Physical = } 94 \text { (Lucente, Fals- } \\
\text { Stewart, Richards, \& Goscha, 2001) }\end{array}$ & \\
\hline & & $\begin{array}{l}\text { The } 39 \text {-item perpetration scale } \\
\text { includes } 5 \text { subscales that measure } \\
\text { physical assault, psychological } \\
\text { aggression, sexual coercion, } \\
\text { negotiation, and injury between } \\
\text { partners. }\end{array}$ & $\begin{array}{l}\text { Internal consistency }(\text { men } \& \text { women } \\
\text { combined): Sexual coercion }=.87 . \\
\text { Internal consistency }(\text { men } \& \text { women } \\
\text { combined): Psychological } \\
\text { Aggression }=.79 \text {. }\end{array}$ & \\
\hline & $\begin{array}{l}\text { For each scale and } \\
\text { subscale, lifetime and past } \\
\text { experiences of abuse can } \\
\text { be obtained. }\end{array}$ & $\begin{array}{l}\text { The physical assault subscale } \\
\text { includes } 12 \text { items that can be } \\
\text { grouped into two categories: minor } \\
\text { and severe. }\end{array}$ & $\begin{array}{l}\text { Evidence of convergent, discriminant } \\
\text { and factorial validity. }\end{array}$ & \\
\hline
\end{tabular}


Table 2 (continued)

\begin{tabular}{|c|c|c|c|c|}
\hline Scale assessment & Construct & Characteristics* & Psychometrics & Developer \\
\hline $\begin{array}{l}\text { Conflict Tactic } \\
\text { Scale-Adult Recall } \\
\text { (CTS2-CA) }\end{array}$ & $\begin{array}{l}\text { To assess HCS past or } \\
\text { present parental behavior } \\
\text { toward each other as } \\
\text { childhood risk factors, } \\
\text { specifically exposure to } \\
\text { violence between parents } \\
\text { and caregivers. }\end{array}$ & $\begin{array}{l}\text { This } 62 \text {-item scale is based on the } \\
\text { CTS } 2 \text { used as adults recalling } \\
\text { behavior of their parents toward } \\
\text { each other - this version of the } \\
\text { CTS } 2 \text { excludes the sexual coercion } \\
\text { scale. It measures an individual's } \\
\text { exposure to three tactics used in } \\
\text { parental interpersonal conflict: } \\
\text { reasoning, verbal aggression and } \\
\text { physical violence. } \\
\text { An } 8 \text {-point Likert scale ranging } \\
\text { from } \\
0 \text { (this has never happened before) } \\
\text { to } 6 \text { (more than 20x in the past } \\
\text { year); and } 7 \text { (not in the past year, } \\
\text { but it did happened before) } \\
\text { responses. }\end{array}$ & $\begin{array}{l}\text { Cronbach's alphas for this measure } \\
\text { were } .80 \text { for father-to-mother verbal } \\
\text { aggression and } .81 \text { for mother-to- } \\
\text { father verbal aggression (Milletich, et } \\
\text { al. } 2010 .) \text {. } \\
\text { As reported by Straus and Donnelly } \\
\text { (2001), this measure's Cronbach's } \\
\text { alpha ranges from } .41 \text { to } .96 \text { as } \\
\text { different versions of the scale, } \\
\text { particularly the short version, may be } \\
\text { deemed less reliable. }\end{array}$ & $\begin{array}{l}\text { Straus et al. } \\
(1995)\end{array}$ \\
\hline $\begin{array}{l}\text { Short Acculturation } \\
\text { Scale for Hispanics } \\
\text { (SASH) }\end{array}$ & $\begin{array}{l}\text { To assess HCS language } \\
\text { use, preferences regarding } \\
\text { media and social } \\
\text { relationships; as well as } \\
\text { participants' generation, } \\
\text { length of residence in the } \\
\text { United States, age of } \\
\text { arrival, and ethnic self- } \\
\text { identification. }\end{array}$ & $\begin{array}{l}\text { This is a } 12 \text {-item scale used to } \\
\text { identify low and high acculturation. } \\
\text { Each item includes a Likert-type } \\
\text { format response ranging from: } \\
1 \text { (Only Spanish); } 2 \text { (Spanish better } \\
\text { than English); } 3 \text { (Both equally); } \\
4 \text { (English better than Spanish); } \\
5 \text { (Only English). }\end{array}$ & $\begin{array}{l}\text { The alpha coefficient for the } 12 \text { items } \\
\text { was .92. Loading factors on } \\
\text { subscales on Language had an alpha } \\
\text { of .90; Media, of .86; and Social } \\
\text { Relations of .78 (Marin, Sabogal, } \\
\text { Marin, Otero-Sabogal, \& Perez- } \\
\text { Stable, 1987). }\end{array}$ & $\begin{array}{l}\text { Marin et al. } \\
(1987)\end{array}$ \\
\hline
\end{tabular}


Table 2 (continued)

\begin{tabular}{|c|c|c|c|c|}
\hline Scale assessment & Construct & Characteristics* & Psychometrics & Developer \\
\hline $\begin{array}{l}\text { Familial Patriarchal } \\
\text { Beliefs (FPB) }\end{array}$ & $\begin{array}{l}\text { To assess HCS patriarchal } \\
\text { beliefs or machismo } \\
\text { among HCSs. }\end{array}$ & $\begin{array}{l}\text { This is a 5-item, 5-point Likert-type } \\
\text { scale with response ranging from } \\
1 \text { (strongly agree) to } 5 \text { (strongly } \\
\text { disagree). Sample FPB scale } \\
\text { question: "A man has the right to } \\
\text { decide whether or not his } \\
\text { wife/partner/girlfriend should work } \\
\text { outside the home." }\end{array}$ & $\begin{array}{l}\text { Cronbach's alpha values were } .79 \text { for } \\
\text { the English version. }\end{array}$ & $\begin{array}{l}\text { M. Smith } \\
(1990)\end{array}$ \\
\hline $\begin{array}{l}\text { Extent of Religiosity } \\
\text { Scale (ERS) }\end{array}$ & $\begin{array}{l}\text { To measure the level of } \\
\text { religiosity among HCSs. }\end{array}$ & $\begin{array}{l}\text { This is a 3-item scale constructed } \\
\text { specifically to measure the level of } \\
\text { religiosity among Arab men. This is } \\
\text { a 5-point Likert-type scale ranging } \\
\text { from } 1 \text { (very religious) to } 5 \text { (not at } \\
\text { all religious). }\end{array}$ & $\begin{array}{l}\text { Alpha coefficient }=.85 \text { among Arab } \\
\text { men in Israel. To date, this measure } \\
\text { has not been used with an HCS } \\
\text { sample. This scale was placed in the } \\
\text { demographic portion of the survey. }\end{array}$ & $\begin{array}{l}\text { Haj-Yahia } \\
(2003)\end{array}$ \\
\hline
\end{tabular}

Note. * Scale and subscale names in characteristics column are titles that scale authors used. 
Data management. Protection against breach of confidentiality was ensured by assigning identifying numbers for each participant. No names or information that would identify the participants were included in the return packets. The list of potential participants was being accessed by the PI and stored in a safe and locked drawer stored separately from other data that may be accessed by the research team. The handling of the questionnaires and the data were solely done by the PI and the research team who have completed the Collaborative Institutional Training Initiative human-subject training. Data entry and coding referenced participants as numbers and letters and was handled by the PI and one RA who was received appropriate training on data entry. In reporting research data, the PI exercised extreme caution to avoid inadvertently disclosing any identifying information that could be identifiable or linked to any participant; thus, reports of group data on HCSs was safer than case studies, even if the name of the participant had been changed. Dissemination of the study findings did not include any information that would reveal the identity of the participants. Demographic data were presented in cumulative percentages and means for the overall sample.

Recruitment and informed consent. All study procedures were completed in accordance with a protocol approved by the Institutional Review Board for the Protection of Human Subjects (IRB) at Florida International University. Recruitment took place in the Fall of 2012 in two South Florida universities. Participants were recruited with the help of two RAs, each were familiar with each university settings they were assigned. The snowball recruitment method was used to engage potential study participants. A total of 116 participants were recruited with the use of flyers posted within the university settings concerning the project; and personal face-to-face contact. Four participants 
approached the PI verbalizing willingness to participate in the study in the Miami university setting. Collection of data stopped once a total of 120 participants were surveyed. The safety of the participants was always considered in all stages of the research process from the initial contact and interview or data collection. Safety issues are of great concern for IPV at-risk participants, especially if they are solicited by public advertising such as flyers and electronic mail. Participants were HCSs, 18 to 25 years old and living in southeastern Florida. Information pertaining to the study was delivered verbally and via an informed-consent form.

Official consent forms required by most review boards contain description of the study as well information about the researcher. Because participants must always receive a copy of the informed-consent form, this posed a dilemma for both the researcher and the HCSs. For abused HCSs, this form may have been a source of danger if found by the abuser; nevertheless, the researcher's contact information was readily available and included on the consent form. Alternative possibilities include oral consent, if the participant chose this route or giving the HCS an abbreviated consent form containing the smallest amount of information the IRB would permit the individual to copy and keep. A third alternative was to only have one signed copy of the consent available, but kept by the researcher.

The consent form informed prospective participants about the study. It included a request for them to take part in answering a total of seven instruments that measure various definitions, perceptions, and experiences of violence; and that no medical intervention and/or benefit will be gained from participating in the research. 
The PI/RA provided an overview of the process. This included reminders that there is no right or wrong answers, all comments and opinions were welcome, confidentiality about identities and experiences would be maintained, and participants were free to stop completing the questionnaire at any time. The time for each participant to complete the survey packet was 60 minutes, and data collection was held in a classroom or meeting room area in a school of nursing or community center. Participants were encouraged to contact the PI for any question or concern pertaining to but not limited to issues regarding IPV in general; obtaining access, information, and referral sources related to IPV; and results and outcome of the research findings.

\section{Potential Benefits of the Research to Human Subjects and Others}

The study was important for three reasons: First, findings from this study will allow researchers to have a better understanding of how lifetime experiences and exposure to abuse affect HCS perceptions of IPV and relationship attitudes. Second, by using the HCS sample, this study will contribute to the existing body of knowledge in alleviating further transference of the cycle of violence in future intimate relationships among youth and young adults, illustrated by risky behaviors such as engaging in highrisk sexual behaviors, tobacco and illicit drug use, drinking and driving, alcohol abuse, ineffective social skills, and inability to manage anger: actions that are highly correlated with IPV events. Third, this study will also help reduce and eliminate health disparities among ethnic minority populations through accumulation of insights, knowledge, and learned skills related to the prevention of IPV across the lifespan. This study will serve as a foundational program of research for the PI over the next 5 to 7 years. 
There is a need for the development of evidence-based tools, evaluation, and research outcomes sensitive to specific cultural groups as well as the design of community strategies and policies to decrease bias and stereotyping among populations, enhance communication skills, and deliver health teaching through interpreters and other modalities. There was no projected harm that could be anticipated from enrollment in the study.

\section{Importance of the Knowledge Gained}

There is little dispute that understanding IPV is a complex endeavor. While clinical and legal definitions of IPV have guided researchers to find common overarching definitions of IPV, data from exploring its contextual definition unique to HCSs' personal past and present experiences remains limited. From one individual to another and from social scientists to health practitioners and law enforcers, labeling an act as "abusive" varies quite often with opinions about families from different cultures or ethnicities (Malley-Morrison, \& Hines, 2004). Researchers have acknowledged that statistics on IPV and family violence have been based on reported incidents of victimization. Conceptions of IPV and risk appraisal for future victimization can differ dramatically between clinicians and women who experience IPV (Cattaneo, 2007). Furthermore, professionals from different disciplines have different perspectives, and there are few clear guidelines for intervention among disciplines (Magnussen et al., 2004; Tower, 2003, 2006; Wandrei \& Rupert, 2000).

\section{Inclusion of Women and Minorities}

It is well documented that health disparities between the White majority and some racial and ethnic minority populations exists. Additionally, health conditions and 
healthcare needs of women differ from those of men, specifically effects of IPV and IGTV. The overarching goals of Healthy People 2020 are (a) to help individuals of all ages increase life expectancy and improve their quality of life, and (b) to eliminate health disparities among different segments of the population (U.S. Department of Health and Human Services, 2010). In this study, Hispanic/Latino(a) college students who selfidentified as Hispanic and/or Latino(a) were recruited. These individuals were be approached by the PI/RA, and the study objectives, benefits, and informed-consent form were explained. Confidentiality of the data was be ensured, and appropriate referrals were provided. All HCSs included in the study received the same information. Study participants were all between the ages of 18 and 25 .

\section{Limitations}

Burns and Groves (2005) identified two types of limitations in quantitative research: theoretical and methodological. Both are known to weaken the generalizability of research outcomes; therefore, a clear framework is needed to avoid theoretical limitations (Burns \& Groves, 2005). Otherwise, the study design would be weak, thus limiting the integrity of the finding and confining the population to which the findings can be generalized (Burns \& Groves, 2005). Convenience samples limit generalizability. HCSs participating in this study may have responded inaccurately to the questions of the FPB, CTS2-CA, SASH, ERS, CTS2 and the student survey due to recall bias or social desirability bias. HCSs may also not have been familiar with the style of the questions on the FPB, CTS2-CA, SASH, ERS, and CTS2. Timing and the nature of data collection for HCS participants may have created added anxiety and stress. 
Qualitative research can be assessed by four criteria outlined by Lincoln and Guba (1985): credibility, transferability, dependability, and conformability. Credibility was enhanced by collaboration with the participants about data conclusions, but in this study, the collaboration analysis was limited to a purposive subsample of participants. This restricted the ability of HCS participants to review and refine their perspectives.

Transferability may be limited to other HCSs since subsample participants were small in number $(n=20)$. A convenience sample might not have matched the population diversity of other subpopulations of HCSs.

\section{Assumptions}

According to Polit (1996), assumptions are statements that commonly acknowledge the truth about a target population, although not yet confirmed by the researcher. This author also mentioned that to prevent assumptions, researchers should search for resemblance between the sample and population being represented. In this study, the sample was not randomly selected; therefore, it will be unsuitable to generalize the findings to a broader population. However, the following assumptions about the population will be accepted as truth until shown to be untruth:

1. Observational learning (modeling) is a cornerstone of social-learning theory; thus, witnessing domestic violence even without being victimized can have a marked impact on later behavior.

2. Participants responded honestly about their perceptions of their confidence in assessing their lifetime experiences of IPV when completing the questionnaire.

3. The researcher remained objective and fair when conducting the study. 


\section{Discussion}

The mixed-methodological approach has always been the PI's choice for the sole purpose of understanding the contextual meanings and experiences of IPV not captured in quantitative or qualitative research alone. Merging two designs not only provides contextual validation to quantitative methods but also adds meaningful results. As Patton (1990) reiterated, "The intent in using this design is to bring together the differing strengths and non-overlapping weaknesses of quantitative methods (large sample size, trends, and generalization) with those of qualitative methods (small sample, details, in depth)." A concurrent-convergent strategy is selected to use "two different methods in an attempt to confirm, cross-validate, or corroborate findings within a single study" (Steckler, McLeroy, Goodman, Bird, \& McCormick, 1992, p. 3).

Possible methodological alternatives that were considered by the author but later eliminated from the study design, mainly due to study feasibility, available time, and resources were to (a) extend an open invitation for HCS participants to join a follow-up focus group, and (b) use the explanatory sequential design that would start in the collection and analysis of quantitative data, followed by the qualitative phase (Creswell \& Plano Clark, 2011). For the latter, challenges also included issues in securing IRB.

The researcher cannot specify how participants will be selected on the second phase until initial findings are obtained ...The researcher must decide which quantitative results need to be further explained ... and who to sample in the second phase and what criteria to use for participant selection. (Creswell \& Plano Clark, 2011, p. 85) 


\section{Conclusion}

There has been relatively little research on the attitudes, perceptions, and experiences of Latinas/os, particularly college students, toward IPV. The mixedmethodological approach adds understanding in the contextual meanings and experiences of IPV not captured in quantitative or qualitative research alone, and the most popular approach to mixing methods is the convergent design (Creswell \& Plano Clark, 2011). To capture different but complementary data on the same topic (Morse, 1991, p. 122), Risjord, Moloney, and Dunbar (2001) stated:

There are three rationales frequently given for using methodological triangulation. The first is completeness. Quantitative methods can further develop findings derived from qualitative research (and vice versa). The methods complement each other, providing richness or detail that would be unavailable from one method alone.... The second might be called abductive inspiration. As in Fleury's research . . . qualitative research is often used where a phenomenon is poorly understood. ... Qualitative investigation can also help organize quantitative data that has already been gathered or suggest ways new of approaching the phenomenon. The final, and most controversial, rationale for triangulation is confirmation. In its most modest form, qualitative methods can clarify the results of quantitative research, such as apparently inconsistent findings. More tendentiously, qualitative and quantitative results are sometimes thought to support each other. Triangulation would thus yield a stronger result than either method could yield alone. (pp. 44-45) 
In general, the prevalence of IPV on college campuses makes it an important issue that merits greater research attention. With the increasing diversity of the U.S. college population, cross-cultural research would serve to illuminate differences and similarities across and within groups for the purpose of designing campus primary prevention and intervention campaigns. This methodology is also highly useful in synthesizing complementary quantitative and qualitative HCSs data findings to develop a more complete understanding of the phenomenon (Creswell \& Plano Clark, 2011), which is the exploration of perceptions and experiences of IPV among HCSs. 


\section{CHAPTER IV}

\section{RESULTS AND FINDINGS}

\section{Demographic and Background Characteristics}

A sample of 120 Hispanic or Latino students were recruited for the study, and 100\% completed the Hispanic College Students' Relationship Study questionnaire. Of the 120 students, $33(27.5 \%)$ had between 1 to 17 missing responses to the demographics questions, $6(5.0 \%)$ had between one to three missing responses to the CTS2 items, 2 (1.7\%) had two missing responses to the CTS2-CA items, $1(0.8 \%)$ had 1 missing response to a ERS item, and there were no missing responses to the FPB and SASH items.

Details of demographic and background characteristics of the study sample are summarized in Table 3. The average age of the participants was 21.4 years $(S D=2.2)$. Most of the participants, $72(60 \%)$ were born in the United States, $98(81.7 \%)$ were U.S. citizens, $19(15.8 \%)$ permanent residents, and $1(0.8 \%)$ was filing for immigration documents or paper. In terms of languages spoken at home, 33 (27.5\%) spoke English only, 37 (30.8\%) spoke Spanish only, and 48 (40.0\%) spoke both English and Spanish. Regarding socioeconomic status, more than half of the participants $(n=81,67.5 \%)$ were currently employed, and $12(14.8 \%)$ of these 81 students indicated financial dependence on their partner. Twenty-eight of the participants $(23.3 \%)$ reported bringing most of the money into their household, while $12(10 \%)$ indicated that it was their partner, and 67 (55.8\%) indicated it was their parents who brought most money into their household. Of participants who were currently employed, 51 (63\%) had yearly incomes below $\$ 20,000$, and $27(33.3 \%)$ earned $\$ 20,000$ or more per year, with $4(4.9 \%)$ earning $\$ 50,000$ or more. 
There were more female participants $(n=75,62.5 \%)$ than male participants $(n=$ $44,36 \%)$, and $1(0.8 \%)$ did not specify a gender. A majority identified themselves as heterosexual $(n=112,93.3 \%), 7(5.8 \%)$ as either gay, lesbian or bisexual, and $1(0.8 \%)$ did not specify their sexual orientation. Sixty-nine $(n=69,57.5 \%)$ were either currently dating or have a boyfriend or girlfriend, while $42(35.0 \%)$ were not currently dating but were previously in a relationship, and $32(26.7 \%)$ indicated currently living with a partner. The average age when the participants started dating was $17.5(S D=3.0)$, with a range between 10 to 24 years old.

More than half of the participants $(n=68,56.7 \%)$ experienced problems while in a relationship, 47 (39.2\%) did not experience problems, and 5 (4.2\%) did not respond to the question. Of the 68 who experienced relationship problems, more than three-quarters experienced communication problems $(n=53,77.9 \%)$, more than half experienced jealousy or lack of trust $(n=39,57.4 \%), 15(22.1 \%)$ experienced family problems, 13 (19.1\%) financial problems, 7 (10.3\%) abuse, 7 (10.3\%) infidelity or adultery, 4 (5.9\%) sexual problems, 2 (2.9\%) children problems, 2 (2.9\%) other problems, and none experienced mental health problems.

\section{Reliability Estimates for Instruments}

Reliabilities of measures were assessed as seen in Table 4. Nunnally (1978, p. 245) recommends that instruments used in basic research have reliability of about .70 or better. However, according to Kline (1999), when dealing with psychological constructs, values below 0.70 can be expected because of the diversity of the constructs being measured. Note that all, with the exception of the Familiar Patriarchal Belief (FPB) scale, indicated high internal reliability which was consistent with previous studies (Table 2). 
The FPB scale used in this study had an alpha coefficient of .606 indicating low internal reliability (according to Nunnally, 1978) even though item \#5 (women should be protected by law) was reverse-scaled. Item analysis was conducted to decide which item(s) to include or to exclude from the FPB scale. The objective of this action is to select a set of items that yields a summed score that is more strongly related to the construct of interest (gender role) than any other possible set of items. Item $\# 5$ had the lowest corrected item-total correlation, and then item \#4 had the next lowest correlation; therefore, they were candidates for further evaluation. To ensure that item \#4 would still have a low correlation after deleting item $\# 5$, the PI reran the reliability analyses procedure without item $\# 5$ and as expected, item $\# 4$ now had the lowest corrected itemtotal correlation. After examining the FPB scale, the PI concluded that item \#5 differed in context from the other four items in terms of measuring patriarchy but not necessarily gender role per se. Assuming that item $\# 5$ is deleted from the FPB scale; the resulted alpha coefficient increased from .606 to .736. Thus, the closer the Cronbach alpha to 1.0, the greater the internal consistency of the items in the scale. Since the FPB scale historically garnered acceptable reliability scores in previous studies (Table 2), the reliability of .606 was accepted by the PI. 
Table 3

Demographic and Background Characteristics

\begin{tabular}{|c|c|c|c|}
\hline Characteristics & Mean & SD & Range \\
\hline Age & 21.4 & 2.2 & 18 to 25 \\
\hline Years lived in the U.S.* & 12.09 & 6.0 & 1 to 24 \\
\hline \multirow[t]{2}{*}{ Age started dating } & 17.5 & 3.0 & 10 to 24 \\
\hline & $n$ & $\%$ & \\
\hline \multicolumn{4}{|l|}{ Gender } \\
\hline Male & 44 & 36.7 & \\
\hline Female & 75 & 62.5 & \\
\hline No response & 1 & 0.8 & \\
\hline \multicolumn{4}{|l|}{ Sexual Orientation } \\
\hline Straight & 112 & 93.3 & \\
\hline Gay & 2 & 1.7 & \\
\hline Lesbian & 3 & 2.5 & \\
\hline Bi-sexual & 2 & 1.7 & \\
\hline No response & 1 & 0.8 & \\
\hline \multicolumn{4}{|l|}{ Born in the United States } \\
\hline Yes & 72 & 60.0 & \\
\hline No & 46 & 38.3 & \\
\hline No answer & 2 & 0.98 & \\
\hline \multicolumn{4}{|l|}{ Languages spoken at home } \\
\hline English only & 33 & 27.5 & \\
\hline Spanish only & 37 & 30.8 & \\
\hline Other only & 1 & 0.8 & \\
\hline Both English and Spanish & 48 & 40.0 & \\
\hline \multicolumn{4}{|l|}{ Immigration Status } \\
\hline US Citizen & 98 & 81.7 & \\
\hline Permanent resident & 19 & 15.8 & \\
\hline Filing for papers & 1 & 0.8 & \\
\hline Other & 2 & 1.7 & \\
\hline \multicolumn{4}{|l|}{ Current relationship status } \\
\hline Dating & 15 & 12.5 & \\
\hline Have boyfriend/girlfriend & 54 & 45.0 & \\
\hline Not dating, was in a relationship & 42 & 35.0 & \\
\hline No answer & 9 & 0.07 & \\
\hline \multicolumn{4}{|l|}{ Living with partner } \\
\hline No & 83 & 69.2 & \\
\hline Yes & 32 & 26.7 & \\
\hline No answer & 5 & 0.04 & \\
\hline
\end{tabular}




\begin{tabular}{|c|c|c|c|}
\hline Characteristics & $n$ & $\%$ & Range \\
\hline \multicolumn{4}{|l|}{ Employment status } \\
\hline Working & 81 & 67.5 & \\
\hline Not working & 38 & 31.7 & \\
\hline Missing & 1 & 0.008 & \\
\hline \multicolumn{4}{|c|}{ Individual yearly income among working participants } \\
\hline Under $\$ 9,999$ & 26 & 32.1 & \\
\hline$\$ 10,000-\$ 19,999$ & 25 & 30.9 & \\
\hline$\$ 20,000-\$ 29,999$ & 10 & 12.3 & \\
\hline$\$ 30,000-\$ 39,999$ & 10 & 12.3 & \\
\hline$\$ 40,000-\$ 49,999$ & 3 & 3.7 & \\
\hline$\$ 50,000-\$ 59,999$ & 4 & 4.9 & \\
\hline Missing & 42 & 35 & \\
\hline \multicolumn{4}{|l|}{ Financially dependent on partner } \\
\hline Yes & 19 & 15.8 & \\
\hline No & 93 & 77.5 & \\
\hline Missing & 8 & 0.06 & \\
\hline \multicolumn{4}{|c|}{ Person who brings most money into household } \\
\hline Self & 28 & 23.3 & \\
\hline Partner & 12 & 10.0 & \\
\hline Parent(s) & 67 & 55.8 & \\
\hline Disability benefits & 1 & 0.8 & \\
\hline Relative(s) & 7 & 5.8 & \\
\hline Friend(s) & 2 & 1.7 & \\
\hline Other & 2 & 1.7 & \\
\hline None & 1 & 0.8 & \\
\hline \multicolumn{4}{|c|}{ Experienced problems while in relationship } \\
\hline Yes & 68 & 56.7 & \\
\hline No & 47 & 39.2 & \\
\hline No response & 5 & 4.2 & \\
\hline \multicolumn{4}{|c|}{ Type of problems experienced while in a relationship } \\
\hline Communication & 53 & 77.9 & \\
\hline Family & 15 & 22.1 & \\
\hline Mental health & 0 & 0.0 & \\
\hline Children & 2 & 2.9 & \\
\hline Abuse & 7 & 10.3 & \\
\hline Sexual & 4 & 5.9 & \\
\hline Infidelity/Adultery & 7 & 10.3 & \\
\hline Jealousy/Lack of trust & 39 & 57.4 & \\
\hline Financial problems & 13 & 19.1 & \\
\hline Other & 2 & 2.9 & \\
\hline
\end{tabular}

Note. ${ }^{*}$ For students who were born outside the United States. 
Table 4

Cronbach Alphas for Cultural Gender Roles, Adult Recall of Parental IPV, Acculturation, Religiosity, and Past and Current Experiences of IPV Scales

\begin{tabular}{lcc}
\hline \multicolumn{1}{c}{ Scales } & $\begin{array}{c}\text { Cronbach's } \\
\text { alpha, a }\end{array}$ & $\begin{array}{c}\text { Number of } \\
\text { items }\end{array}$ \\
\hline Cultural Gender Roles (FPB) & .606 & 5 \\
Adult Recall of Parental IPV (CTS2-CA) & .947 & 62 \\
Acculturation (SASH) & .909 & 12 \\
Religiosity (ERS) & .913 & 3 \\
Past and Current Experiences of IPV (CTS2) & .961 & 78 \\
\hline
\end{tabular}

\section{Descriptive Findings for Major Study Variables}

Research question 2 stated: What is the level of (a) cultural gender roles; (b) adult recall of parental IPV; (c) acculturation; and (d) religiosity among HCSs?

Descriptive analysis was performed to evaluate whether HCSs perceived themselves as victims/survivors of IPV or perpetrators of IPV while in a relationship. Among 120 participants surveyed, 114 (95\%) participants did not perceive themselves as victims or survivors of IPV, while six (5\%) reported otherwise. Regarding perceptions on whether they do/did not perceive themselves as perpetrators of violence while in a relationship, $115(95.8 \%)$ participants responded no; while five (4.2\%) participants responded yes as perceiving themselves as perpetrators of IPV.

Out of the 120 HCSs participants, an average of $116(96.7 \%)$ answered the CTS2 questionnaire. Respondents' perception response highly differs from their actual CTS2 scores on levels of victimization and levels of perpetration. Respondents indicated that as much as $66 \%(n=73)$ of individuals surveyed were victims/survivors of verbal 
aggression by their intimate partner followed by sexual coercion $(n=37,31 \%)$. Almost $67 \%(n=72)$ participants indicated that they utilized verbal abuse (verbal aggression) followed by sexual coercion $(n=38,32.5 \%)$ to perpetrate violence against their intimate partner.

Table 5

Descriptive Finding on Major Study Variables

\begin{tabular}{lcc}
\hline \multicolumn{1}{c}{ Scales } & $M$ & $S D$ \\
\hline Familial Patriarchal Beliefs (FPB) & 11.0 & 3.1 \\
Extent of Religiosity (ERS) & 8.1 & 3.2 \\
Short Acculturation Scale for Hispanics (SASH) & 40.7 & 8.7 \\
Conflict Tactics Scale - Adult Recall (CTS2-CA) & 155.6 & 122.2 \\
*Revised Conflict Tactics Scale (CTS2) & 193.3 & 175.9 \\
\hline
\end{tabular}

*DV; measures overall level of victimization and level of perpetration.

Cultural gender role. The level of cultural gender roles was measured by the Familial Patriarchal Belief (FPB) scale. The average total FPB score was $11.0(S D=3.1)$, and scores ranged from 5 to 25 . A majority of the participants either disagreed or strongly disagreed on the first four items in the Familial Patriarchal Beliefs instrument. One hundred and six (88\%) either disagreed or strongly disagreed with the statement that a man has the right to decide whether or not his wife/partner/girlfriend should work outside the home, $104(87 \%)$ disagreed or strongly disagreed that a man has the right to decide whether his wife/partner/girlfriend should go out in the evening with her friends, 76 (63\%) disagreed or strongly disagreed that it is sometimes important for a man to show his wife/partner/girlfriend that he is head of the house, and 109 disagreed or strongly 
disagreed that a man has the right to have sex with his wife/partner/girlfriend when he wants, even though she may not want to. In contrast, most of the participants $(n=104$, $87 \%$ ) either agree or strongly agree that women should be protected by law if their partners beat them.

Table 6

Descriptive Finding on Dependent Variables: Perceived Victimization and Perceived Perpetration

Total $N^{*} \quad n \quad \%$

Perceptions of IPV

Perceived self as victim/survivor of IPV

120

6

5

Perceived self as perpetrator of IPV while in a

120

5

4 relationship

Note. *Total number of respondents.

A Mann-Whitney $U$ test was conducted to evaluate gender differences and gender role. The results of the test were significant, $z=-3.158, p=.002$. Female participants had an average rank of 52.49, while male participants had an average rank of 72.81 . Thus, it is important to note that female participants score lower, on the average, than male participants on gender role measures.

Adult recall of parental IPV. Adult recall of parental IPV was measured by the Revised Conflict Tactics Scale - Adult Recall (CTS2-CA) scale. The mean CTS2-CA score was $110(S D=87.6)$ with a minimum score of 0 and maximum of 654 . The subscale with the highest average was negotiation $(M=110.4, S D=87.6)$, followed by verbal aggression $(M=36.1, S D=51.3)$. The subscale with the lowest score was injury between partners $(M=4.9, \mathrm{~S} D=20.6)$. 
Table 7

Mean Gender Role Ratings of Male and Female Hispanic College Students

\begin{tabular}{|c|c|c|c|c|}
\hline \multirow{2}{*}{ Belief } & \multicolumn{2}{|c|}{ Male Respondents } & \multicolumn{2}{|c|}{ Female Respondents } \\
\hline & $M$ & $S D$ & $M$ & $S D$ \\
\hline $\begin{array}{l}\text { A man has the right to decide } \\
\text { whether or not his } \\
\text { wife/partner/girlfriend should work } \\
\text { outside the home }\end{array}$ & 1.77 & 1.10 & 1.20 & 0.49 \\
\hline $\begin{array}{l}\text { A man has the right to decide } \\
\text { whether or not his } \\
\text { wife/partner/girlfriend should go } \\
\text { out in the evening with friends }\end{array}$ & 1.86 & 1.13 & 1.27 & 0.56 \\
\hline $\begin{array}{l}\text { Sometimes it is important for a man } \\
\text { to show his wife/partner/girlfriend } \\
\text { that he is the head of the house }\end{array}$ & 2.83 & 1.43 & 1.93 & 1.10 \\
\hline $\begin{array}{l}\text { A man has the right to have sex } \\
\text { with his wife/partner/girlfriend } \\
\text { when he wants, even though she } \\
\text { may not want to }\end{array}$ & 1.59 & 1.17 & 1.12 & 0.54 \\
\hline $\begin{array}{l}\text { Women should be protected by law } \\
\text { if their partners beat them }\end{array}$ & 4.20 & 1.41 & 4.64 & 1.10 \\
\hline
\end{tabular}

Short acculturation scale for Hispanics. Acculturation was measured with the Short Acculturation Scale for Hispanics (SASH). The mean SASH score was 40.7 (SD = $8.7)$, with a minimum of 22 and maximum of 60 . Seventy-four (62\%) read and spoke either English only or English better than Spanish, 33 (27.5\%) read and speak English and Spanish equally, and 13 (11\%) read and speak Spanish only or Spanish better than English. As a child, 33 (28\%) used only English or English better than Spanish, 31 (26\%) used English and Spanish equally, and 56 (47\%) used only Spanish or Spanish better than English. 
Regarding social group preference (Table 7), 58 (48\%) of the participants indicated that their close friends consisted of more Latinos than Americans, or all Latinos, $36(30 \%)$ had about equal numbers of each, and $26(22 \%)$ had more Americans than Latinos or all Americans.

Table 8

Preferred Ethnicity of Social Groups

\begin{tabular}{lccccc}
\hline Social groups & $\begin{array}{c}\text { All Latinos/ } \\
\text { Hispanics }\end{array}$ & $\begin{array}{c}\text { More Latinos } \\
\text { than Americans }\end{array}$ & About equal & $\begin{array}{c}\text { More } \\
\text { Americans } \\
\text { than Latinos }\end{array}$ & $\begin{array}{c}\text { All } \\
\text { Americans }\end{array}$ \\
\hline $\begin{array}{l}\text { Close friends } \\
\begin{array}{l}\text { People in } \\
\text { social }\end{array}\end{array}$ & $5(4.2 \%)$ & $53(44.2 \%)$ & $36(30 \%)$ & $22(18.3 \%)$ & $4(3.3 \%)$ \\
$\begin{array}{l}\text { gatherings } \\
\begin{array}{l}\text { Persons who } \\
\text { visit }\end{array}\end{array}$ & $6(5 \%)$ & $52(43.3 \%)$ & $46(38.3 \%)$ & $14(11.7 \%)$ & $2(1.7 \%)$ \\
$\begin{array}{l}\text { Children's } \\
\text { friends }\end{array}$ & $0(0 \%)$ & $7(5.8 \%)$ & $102(85 \%)$ & $8(6.7 \%)$ & $3(2.5 \%)$ \\
\hline
\end{tabular}

Extent of religiosity. Religiosity was measured with the Extent of Religiosity Scale (ERS). The mean level of religiosity was $8.1(S D=3.2)$ with a minimum of 3 and maximum of 15 . The ERS measured the extent of religiosity of participants with assigned scale of 1 (very religious) through 5 (not at all religious). Participants' extent of practicing and adhering to laws and customs of their religion as well as participants' sense of affiliation with their religion was measured with assigned scale of 1 (all the time) through 5 (never). The largest proportion of participants considered themselves as somewhat religious 56 (46.7\%), while 39 (32.5\%) considered themselves as either not religious or not at all religious, and 25 (20.8\%) considered themselves as either religious 
or very religious. Forty-nine (40.8\%) rarely or never practiced and adhered to laws and customs of their religion, while 41 (34.2\%) practiced or adhered sometimes, and 30 (25\%) practiced or adhered either most of the time or all the time. Fifty-five (25.9\%) rarely or never identified or felt affiliated with their religion, 32 (26.7\%) sometimes, and $32(26.7 \%)$ identified or felt affiliated with the religion most of the time or all of the time.

\section{Hypotheses}

In this section, each hypothesis is reported and results follow. Each hypothesis was tested at the .05 level of significance.

Hypothesis 1. Hypothesis 1 predicted that perceptions of IPV would be directly related to gender, cultural gender roles, adult recall of parental IPV, acculturation, religiosity, level of victimization, and level of perpetration. Perceptions of IPV were measured using two questions answerable by yes or no. As shown in Table 6 , only $5 \%$ of participants perceived themselves as victims and only $4.2 \%$ perceived themselves as perpetrators.

Gender and perceptions of IPV. Among 120 participants surveyed, 114 (95\%) participants did not perceive themselves as victims or survivors of IPV, while six (5\%) reported otherwise. Regarding perceptions on whether they do/did not perceive themselves as perpetrators of violence while in a relationship, 115 (95.8\%) participants responded no, while five (4.2\%) participants responded yes to perceiving themselves as perpetrators of IPV. Categorical analysis of the data was done to determine whether there was any association between gender and perceptions of victimization. Forty-three (36.1\%) of male participants reported that they did not consider themselves as a victim/survivor of a partner or dating violence, while one $(0.8 \%)$ reported that he did. 
Seventy (58.8\%) of female participants did not perceived themselves as a victim/survivor of a partner or dating violence, while five (4.2\%) reported that she did. To test the association between gender, a categorical variable, and perceived victimization of IPV, chi-square analysis was used. No relationship was found between gender and perceived level of victimization, $\chi^{2}(1, N=120)=1.12, p=.29$. Both men and women were equally likely to perceive themselves as victims of IPV.

Forty-two (35.3\%) of the male participants reported that they did not consider themselves as perpetrator of violence while in a relationship, while two (1.7\%) reported that he was. Seventy-two (60.5\%) of the female participants did not perceived themselves as perpetrator of violence while in a relationship, while three (2.5\%) reported that they were. To test the association between gender, a chi-square test was performed, and no relationship was found between gender and perceived perpetrator of IPV, $\chi^{2}(1, N=119)$ $=.020, p=.886$. The $p$ value indicated that there was no significant association between gender and perceived perpetrator of IPV; both men and women are equally likely to perceive themselves as perpetrators of IPV. The findings of the study did not support the hypothesis that gender is related to perceptions of IPV.

Bivariate correlational analyses to test relationships between the interval level independent variables (i.e., FPB, CTS2CA, SASH and ERS) and the dependent variables (i.e., perceived victimization and perceived perpetration of IPV). The purpose of these analyses was to identify the strength and direction of relationships between variables.

Cultural gender roles and perceptions of IPV. A Pearson product-moment correlation was computed between FPB scores and victimization. The first hypothesis postulated that perceptions of IPV are directly related to patriarchal beliefs and perceived 
victimization. The correlations between patriarchal beliefs and perceived IPV victimization did not yield a linear relationship with $r=-.052, p=.570$ which indicated that there was no significant association between cultural gender roles and perceived victimization. A correlation was computed between patriarchal beliefs and perpetration. The first hypothesis proposed that patriarchal beliefs and perceived perpetration would be directly related. The correlation between patriarchal beliefs and perceived IPV perpetration yield a linear relationship with $r=.258, p=.004$ which indicated that there was significant association between cultural gender roles and perceived perpetration of IPV. This finding of the study supported the hypothesis that patriarchal beliefs is directly related to perceived perpetration of IPV.

Adult-recall of parental IPV and perceptions of IPV. The first hypothesis also postulated that there would be a relationship between adult-recall of parental IPV and perceived victimization. The correlations between adult-recall of parental IPV and perceived IPV victimization indicated that there was not a significant relationship $(r=-$ $.016, p=.885)$. The finding of the study did not support the hypothesis that adult recall of IPV was related to perceived victimization of IPV. However, the correlation between adult-recall of parental IPV and perceived IPV perpetration yielded a significant linear relationship with $r=.408, p=.000$. Thus, the findings of the study partially supported the hypothesis that adult-recall of parental IPV is related to perceived IPV.

Acculturation and perceptions of IPV. The first hypothesis further postulated that there would be a relationship between acculturation and perceived victimization. The correlation between acculturation and perceived IPV victimization yielded a significant linear relationship with $r=.229, p=.012$. The correlation between acculturation and 
perceived IPV perpetration did not yield a linear relationship with $r=-.027, p=.771$.

This finding of the study supported the hypothesis that acculturation is related to perceived victimization of IPV. However, it did not support the hypothesis that acculturation is related to perceived perpetration of IPV.

Religiosity and perceptions of IPV. The first hypothesis also predicted that there would be a relationship between extent of religiosity and perceived victimization. The correlation between extent of religiosity and perceived IPV victimization did not yield a linear relationship with $r=-.029, p=.750$. The correlation between religiosity and perceived IPV perpetration did not yield a linear relationship with $r=-.070, p=.446$. The findings of the study did not support the hypothesis that religiosity is directly related to perception of IPV .

Level of victimization and level of perpetration and perceptions of IPV. Prior to evaluating if perceived victimization and perceived perpetration of IPV are related to levels of victimization and perpetration (CTS2), descriptive analyses were done. One hundred forty-four (95\%) participants did not perceive themselves as victims or survivors of IPV, while six (5\%) reported otherwise. Regarding perceptions on whether they do/did not perceive themselves as perpetrators of violence while in a relationship, $115(95.8 \%)$ participants responded no, while five (4.2\%) participants responded yes to perceiving themselves as perpetrators of IPV. Almost two-thirds $(n=73,66 \%)$ of individuals surveyed were victims/survivors of verbal aggression by their intimate partner followed by sexual coercion $(n=37,31 \%)$. Almost $67 \%(n=72)$ participants indicated that they utilized verbal abuse (i.e., verbal aggression) followed by sexual coercion $(n=38$, $32.5 \%$ ) to perpetrate violence against their intimate partner. 
The first hypothesis also predicted that there would be a relationship between level of victimization and level of perpetration with perceived victimization and perceived perpetration. The correlation between HCSs' level of victimization and perceived victimization yield a significant linear relationship with $r=.381, p=.000$; as well as with level of victimization and perceived perpetration with $r=.271, p=.003$. The correlation between HCSs' level of perpetration and perceived victimization also yielded significant values of $r=.491, p=.000$; as well as for HCSs' level of perpetration and perceived perpetration with values of $r=.561, p=.000$.

These findings supported the first hypothesis that there would be a relationship between level of victimization and level of perpetration with perceived victimization and perceived perpetration.

Hypothesis 2. It was proposed that there would be relationships between gender, cultural gender roles, adult recall of parental IPV, acculturation and religiosity, with level of victimization (CTS2). For the second hypothesis, SPSS was used to test associations between independent variables and level of victimization. Since gender is a categorical variable, nominal measures were used; the Chi-Square test was conducted to determine any significant association with level of victimization. To assess the relationship between level of victimization (interval level variables) and gender, frequency scores of level of IPV victimization were computed from the responses to CTS2 questions according to the CTS2 scoring guidelines by Straus and colleagues (2003). Level of victimization were assessed by creating dichotomous variables following the prevalence method in the scoring guideline, where a score of 1 indicates one or more acts of violence, and a score of 0 indicates there were no acts of violence (experiences of IPV). 
Bivariate correlational analyses were used to test relationships between the interval level independent variables (i.e., FPB, CTS2CA, SASH and ERS) and the dependent variables (level of victimization).

Gender and experiences (level) of victimization. In terms of assault severity by a partner, thirteen female (17.3\%) participants indicated that they were victims of minor assault; while $16(21 \%)$ participants indicated that they were victims of major assault by an intimate partner. Fifteen (34\%) among male participants reported minor assault; while nine (20\%) male participants reported that they were victims of major assault by an intimate partner. A Chi-Square test was conducted between gender and dichotomized level of victimization to determine any significant association. Results yield, Pearson $\chi^{2}$ $(1, N=119)=.645, p=.422$. The $p$-value indicated that there was no significant association between gender and experiences of victimization on combined subscale scores on CTS2. This can be interpreted that both men and women are equally likely to be victims of IPV. The findings of the study did not support the hypothesis that gender is related to HCSs' level of victimization.

Cultural gender role and level of victimization. A Pearson product-moment correlation was computed between FPB scores and level of victimization. The second hypothesis postulated that cultural gender roles are directly related to and level of victimization. The correlation was not significant at 0.05 , with $r=.164, p=.127$. The findings of the study did not supported the hypothesis that cultural gender role is related to HCSs' level of victimization.

Adult recall of parental IPV and level of victimization. The second hypothesis further postulated that there would be a relationship between adult-recall of parental IPV 
and level of victimization. The correlation was significant at 0.05 , with $r=.267, p=.027$. The findings of the study supported the hypothesis that adult-recall is related to HCSs' level of victimization.

Acculturation and level of victimization. The second hypothesis also stated that there would be a relationship between acculturation and level of victimization. The correlation between acculturation and level of victimization did not yield a significant linear relationship. The correlation was not significant at 0.05 , with $r=.267, p=.275$. Thus, the findings of the study did not supported the hypothesis that acculturation is related to HCSs' level of victimization.

Religiosity and level of victimization. The second hypothesis also predicted that there would be a relationship between religiosity and level of victimization. The correlation was not significant at 0.05 , with $r=.086, p=.430$. The findings of the study did not supported the hypothesis that religiosity is related to HCSs' level of victimization.

The findings on the second hypothesis are only partially supported. Adult -recall of IPV among HCSs was the only independent variable that was related to HCSs' level of victimization and the only independent variable that supported Hypothesis 2 (Table 9).

Hypothesis 3. It was proposed that there would be relationships between gender, cultural gender roles, adult recall of parental IPV, acculturation and religiosity, with level of perpetration (CTS2). Since gender is a categorical variable, nominal measures were used; the Chi-Square test was conducted to determine any significant association with level of perpetration. To assess the relationship of level of perpetration (interval level variables) and gender, frequency scores of level of perpetration were computed from the responses to CTS2 questions according to the CTS2 scoring guidelines by Straus and 
colleagues (2003). Level of IPV perpetration were assessed by creating dichotomous variables following the prevalence method in the scoring guideline, where a score of 1 indicates one or more acts of violence perpetrated towards an intimate partner, and a score of 0 indicates there were no acts of violence perpetrated towards an intimate partner (experiences of IPV).

Bivariate correlational analyses were used to test relationships between the interval level independent variables (i.e., FPB, CTS2CA, SASH and ERS) and the dependent variables (level of perpetration). For the third hypothesis, SPSS was used to test associations between independent variables and level of perpetration. Again, since gender is categorical variable, nominal measures are used, the Chi-Square test was conducted to determine any significant association with experiences of IPV perpetration and Pearson's $R$ was used for the rest of the independent variables.

Gender and experiences (level) of perpetration. In terms of assault severity by self to an intimate partner, fifteen (20\%) female participants indicated that they were perpetrators of minor assault; while $13(17 \%)$ participants indicated that they were perpetrators of major assault to an intimate partner. Among male participants, 14 (32\%) reported minor assault; while seven (16\%) male participants reported that they were perpetrators of major assault to an intimate partner. A Chi-Square test was conducted to determine any significant association with experiences of perpetration with results that yield Pearson $\chi^{2}(1, N=119)=1.118, p=.290$. There was no significant association between gender and level of perpetration between men and women; thus, both men and women are equally likely to be perpetrators of IPV on combined subscale scores on CTS2. 
Cultural gender role and level of perpetration. A Pearson product-moment correlation was computed between FPB scores and level of perpetration. The third hypothesis postulated that cultural gender roles are directly related to and level of victimization. The correlation was not significant at 0.05 , with $r=.068, p=.531$. Thus, this finding of the study did not supported the hypothesis that cultural gender role is related to HCSs' level of perpetration.

Adult recall of parental IPV and level of perpetration. The third hypothesis further postulated that there would be a relationship between adult-recall of parental IPV and level of perpetration. The correlation was not significant at 0.05 , with $r=.173, p=$ .156. The findings of the study did not supported the hypothesis that adult-recall is related to HCSs' level of perpetration.

Acculturation and level of perpetration. The third hypothesis also stated that there would be a relationship between acculturation and level of perpetration. The correlation between acculturation and HCSs' level of perpetration did yield a significant linear relationship. The correlation was significant at 0.05 , with, $r=.219, p=.041$. This finding of the study supported the hypothesis that acculturation is related to HCSs' level of perpetration.

Religiosity and level of perpetration. The third hypothesis also predicted that there would be a relationship between religiosity and level of perpetration. The correlation was not significant at 0.05 , and with $r=.058, p=.590$. The findings of the study did not supported the hypothesis that religiosity is related to HCSs' level of perpetration. 
Thus, the hypothesis that there are relationships between gender, cultural gender roles, adult recall of parental IPV, religiosity, and level of perpetration was minimally supported by study findings. The independent variable acculturation was the only variable that supported the hypothesis (Table 10).

Table 9

Correlations Between Cultural Gender Roles, Adult Recall of Parental IPV, Acculturation, Religiosity Scales and Level of Victimization

\begin{tabular}{lcc}
\hline & $r$ & $p$ \\
\hline Gender Role (FPB) & 0.164 & 0.127 \\
& & \\
Adult Recall of Parental IPV (CTS2-CA) & 0.267 & $0.027^{*}$ \\
Acculturation (SASH) & 0.120 & 0.275 \\
Religiosity (ERS) & 0.086 & 0.430 \\
\hline
\end{tabular}

*. Significant at $<.05$ level.

Table 10

Correlations Between Gender, Cultural Gender Roles, Adult Recall of Parental IPV, Acculturation, Religiosity Scales and Level of Perpetration

\begin{tabular}{lcc}
\hline & $r$ & $\rho$ \\
\hline Gender Role (FPB) & 0.07 & 0.531 \\
Adult Recall of Parental IPV (CTS2-CA) & 0.17 & 0.156 \\
Acculturation (SASH) & 0.22 & $0.041^{*}$ \\
Religiosity (ERS) & 0.06 & 0.590 \\
\hline
\end{tabular}

*. Significant at $<.05$ level. 
Hypothesis 4. Hypothesis 4 postulated that there would be relative contributions of cultural gender roles, adult recall of parental IPV, acculturation, and religiosity on level of victimization.

To test this research hypothesis, a regression analysis was carried out in SPSS with cultural gender roles, adult-recall of parental IPV, acculturation, and religiosity as independent variables and level of victimization as dependent variable. Prior to running the relevant linear regression analysis, the independent variables, cultural gender roles, adult-recall of parental IPV and religiosity, and the dependent variable, level of victimization were transformed using the square-root transformation (Marcus, Lindahl \& Neena, 2001). Pairwise deletion (SPSS default) was used deal with missing data. Although the procedure cannot include a particular variable when it has a missing value, it can still use the case when analyzing other variables with non-missing values. This was followed by a regression analysis (ENTER method) carried out in SPSS.

Multiple regression analysis was used to test for relative contributions or to predict the values on independent variables cultural gender roles, adult recall of parental IPV, acculturation and religiosity to dependent variable level of victimization. A multiple linear regression analysis was run, and results on Table 11 indicated that CTS2-CA was a significant predictor of level of victimization. Of the five independent variables, the square root of adult recall was significantly associated with the square root of level of perpetration $(\beta=0.177, S E=0.85, p=0.041)$. The overall regression model, however, was not statistically significant $(F=1.499, p=0.201)$. 
Hypothesis 5. Hypothesis 5 predicted that there would be relative contributions of cultural gender roles, adult recall of parental IPV, acculturation, and religiosity to levels of perpetration.

To test this hypothesis, regression analysis was carried out in SPSS with cultural gender roles, adult-recall of parental IPV, acculturation, and religiosity as independent variables and level of perpetration as dependent variable. Prior to running the relevant linear regression analysis, the independent variables, cultural gender roles, adult-recall of parental IPV and religiosity, and the dependent variable, level of perpetration were transformed using the square-root transformation. Pairwise deletion was used deal with missing data. This was followed by a regression analysis (ENTER method) carried out in SPSS. The regression was not significant at alpha $=0.05$ (see Table 12).

Table 11

Regression Analysis of Level of Victimization Predicted by Gender, Cultural Gender Roles, Adult Recall Of Parental IPV, Acculturation, and Religiosity

\begin{tabular}{lcccc}
\hline Independent Variables & $B$ & $S . E$. & $T$ & $p$-value \\
\hline Intercept & -3.390 & 4.7 & -.695 & .489 \\
Gender & .305 & .996 & .306 & .760 \\
Cultural Gender Role & 2.420 & 3.9 & .627 & .533 \\
Adult recall & \\
Acculturation & .199 & .086 & 2.308 & $.024^{*}$ \\
Religiosity $^{\#}$ & .057 & .049 & 1.149 & .254 \\
\hline
\end{tabular}

* Significant at $<.05$ level.

${ }^{\#}$ Values reported are for the square root transformations of independent variables.

Note. Regression model: R-square $=0.093, F=1.530, p=0.191$. Data shown are unstandardized coefficients. 
Table 12

Regression Analysis on Level of Perpetration predicted by Gender, Cultural Gender Roles, Adult Recall Of Parental IPV, Acculturation, and Religiosity $(N=120)$

\begin{tabular}{|c|c|c|c|c|}
\hline $\begin{array}{l}\text { Independent } \\
\text { Variables* }\end{array}$ & $B$ & S.E. & $T$ & $p$-value \\
\hline Intercept & -2.595 & 4.660 & -.557 & .579 \\
\hline Gender (Male) & -.165 & 1.001 & -.165 & .869 \\
\hline Cultural Gender Role & 2.883 & 3.796 & .760 & .450 \\
\hline Adult recall ${ }^{\#}$ & .177 & .085 & 2.077 & .051 \\
\hline Acculturation & .065 & .048 & 1.359 & .178 \\
\hline Religiosity $^{\#}$ & .018 & .775 & .023 & .982 \\
\hline
\end{tabular}

${ }^{\#}$ Values reported are for the square root transformations of independent variables.

Note. Data shown are unstandardized coefficients. R-square $=0.092, F=1.499, \mathrm{p}=0.201$

\section{Qualitative Findings}

In an effort to have a better understanding of HCSs' perceptions of IPV, a subsample of 20 participants was randomly selected to answer the following four open ended questions. Age range for the subsample was from $18-25$ years old $(M=22.05 ; S D$ $=2.35)$. There were more female participants $(n=13,65 \%)$ than males $(n=7,35 \%)$. Most of the subsample participants, $15(75 \%)$ were born in the United States, while four $(20 \%)$ were born in Cuba and one (5\%) stated "other." In terms of languages spoken at home, 5 (25.0\%) used English only, 4 (20.0\%) used Spanish only, and 9 (45.0\%) used both English and Spanish. For the first three questions, the participants were asked to provide at least one example. Participants surveyed answered either with short sentences and phrases as exemplified below.

1. What does intimate partner violence mean to you? 
Twelve of the 20 participants indicated that IPV meant use of force in order to gain control toward an intimate partner by yelling and being verbally abusive such as "do something that they don't want to," "beating you... force them to have sex," "forcing to do sexual acts" and "hurting them in either a physical or mental sense by forcing them to do something they don't want to do." Four participants viewed IPV as acts perpetrated with intentions to hurt an intimate partner by "hurting physically" such as "struck you with something," "beating you," "choke, grab, slaps, burns, kicks any physical harm." These participants gave slapping, pushing and shoving as additional examples. Two participants viewed IPV overlapping acts of violence to be "hurting someone in a physical, verbal and mental" way such as "when someone is hurt or verbal abuse when a partner is constantly belittled" and "beating, lying constantly, hurting feelings on purpose." Two participants indicated that IPV includes acts in which couples are in "mutual agreement of violence during intimacy." Contrary to above statements, one participant stated that "Rough sex is good", while another stated, "It means that both partners are violent, but they know what they are doing--not really trying to hurt one another. Statements made by the two latter students included "Submissive and dominance," and "Partners kissing and male slams female against the wall."

2. What do you think intimate partner violence means to your parents?

Eight of the 20 participants perceived that their perceptions of IPV were similar to their parents. Five out of the eight participants responded "Same as with parents." One stated "Probably same as with me," while another stated "I think my parents would agree with the statements I provided above." Another participant reported "I think it means the same thing from what I wrote above." Ten participants perceived that the meaning of IPV 
for parents included acts of verbal fights, rape and other physical manifestations of IPV. One participant reported, "To my parents, violence is something that damages the partner either physical or mental." Likewise, another participant stated "To my parents, it probably includes physical violence where someone is hurt and abused" such as "throwing shoes at each other, typical Cuban household." Another participant elaborated:

Physical abuse. When I was little, they [parents] did turn to physical violence to get point across but they 'defended' themselves from each other. My mother also is in another physical abusive relationship but was able to defend herself from getting hurt.

One participant responded "I honestly don't think my parents have a clue. They are from Gen X--extremely Old School, uneducated. I wouldn't be able to give you an example." Another participant stated: "They love it. I grew up having my parents having violent sex and personally that was the way I was raised."

3. Do you believe that your definition of intimate partner violence is similar to that of your parents? Why?

Fifteen of the 20 participants reported that their definitions of IPV are similar to that of their parents. Examples of participants' statements were: "Yes, because they are similar situation," "I believe they are similar, but I have a broader range of what partner violence is while my parents' viewpoint may only be isolated to physical violence," "Yes, I feel they are similar because it's the same no matter what," and "Yes, because they taught me." Four participants, however, disagreed, stating "No! Because violence today and the violence years ago are looked upon differently," "No way," "No because to 
me it's intimacy as in sex but since they are more old fashioned, it's verbal abuse to them that would be more common," and another participant responded, "I think that my definition of intimate partner violence is different because mine includes more variability since I believe that violence comes in different ways, not just one.” Finally, one participant, who also disagreed whether his/her definition of IPV is similar to that of his/her parents stated, "No, because I believe some people enjoy physical abuse when intimate, and I feel my parents won't agree with that."

4. Tell me about what kinds of violence there are.

The fourth question produced descriptions of five types of violence. Although not required, more than half of the participants surveyed also gave their own examples: (a) physical abuse and assault, (b) emotional due to verbal aggression, (c) psychological and mental, (d) social, and (e) sexual. Eighteen of the 20 participants overwhelmingly agreed and responded that physical abuse and assault are indeed forms of IPV. Examples that respondents gave were: "being aggressive," "hitting, slapping, raping," "explosive acts," and "punching." Ten of the students viewed emotional abuse as a type of violence which was exemplified by the following statements: "Messing up with the emotions," and "making the other person feel guilty which makes them do what they want." Nine participants also stated that verbal abuse is a form of IPV. Examples that were given are: "taunting" and "belittling," calling the person names, insulting, swearing." Nine participants also perceived that mental / psychological abuse is a form of IPV; examples were "being spiteful "and "manipulating acts." Two participants commented that social abuse includes bullying. As far as sexual violence, only participant identified it as a form of IPV. 


\section{Chapter Summary}

Statistical analyses used for this study were simple descriptive analysis to measure variability on gender, cultural gender roles, adult recall of parental IPV, acculturation, and religiosity among HCSs. Correlational techniques (i.e., Chi Square and Pearson's $R$ ) were used to determine the level of relationships that exist between variables; and multiple regressions to predict the relative contributions of gender, cultural gender roles, adult recall of parental IPV, acculturation, and religiosity to level of victimization and level of perpetration.

The results from the data analyses in this mix method research indicated that there were significant discrepancies among HCSs on how they perceive victimization and perpetration while in a relationship and to their self-report levels of victimization and perpetration based on their CTS2 scores. Both men and women were equally likely to be victims and perpetrators of IPV. Patriarchal beliefs was directly related to perceived perpetration of IPV but not perceived IPV victimization. Acculturation was closely associated with perceived victimization; while cultural gender role and adult recall of parental IPV were associated with perceived perpetration.

Adult recall of parental IPV was the only independent variable that is related to HCSs' level of victimization; while acculturation was the only variable that was related to HCSs' level of perpetration (based on CTS2 scores). Regression analyses indicated that adult recall of parental IPV was a significant predictor on the level of victimization but not on the level of perpetration. Qualitative analysis of the data indicated some level of IGTV as well as some insight on HCSs' disparity on perceptions of IPV. 


\section{CHAPTER V}

\section{DISCUSSION, IMPLICATIONS, AND CONCLUSIONS}

\section{Overview of the Study}

This mixed methods study was designed to explore IPV as seen through the eyes of Hispanic college students, young men and women between the ages of 18 and 25 . The participants in the study were 120 college students attending two South Florida universities, both serving diverse student populations. A subsample of 20 participants was randomly chosen to provide qualitative responses to complement the quantitative analysis. Women comprised close to two-thirds of the participants. Twenty-one years was the average age for all participants.

Almost two thirds of the participants were born in the United States, and the overwhelming majority was U.S. citizens. The remaining participants were all permanent residents, with the exception of one student who was in the process of filing for immigration papers. Close to half of the participants spoke both English and Spanish at home. English and Spanish, respectively, were the sole home languages of $27.5 \%$ and $30.8 \%$ of the participants.

More than half the participants were currently dating or reported having a boyfriend or girlfriend, and over a third were not currently dating but previously been in a relationship. Slightly more than one-quarter of the participants were living with a partner. On the average, the participants began dating in high school, at the age of 17-18 years. However, there was substantial variation among individual participants; the age at onset of dating ranged from 10 years to 24 years. 
Roughly two-thirds of the participants were employed. For more than half these students, their parents brought the most money into the household. About an eighth of participants reported that they were financially dependent upon their partner. The proportion of participants who indicated that they brought in more money than their partner was more than double that who reported the reverse (23.3\% versus $10 \%)$. The majority of household income was provided by HCSs' parent(s), followed by the students themselves who either had full-time or part-time jobs. This study did not assess the difference of financial dependence on a partner, or alternately, being the one who brings home more money, in the incidence of IPV. However, financial dependence is a documented impediment to leaving an abusive relationship and, indeed, preventing a partner from working is a frequently used technique by abusers to maintain control (McCloskey et al., 2007; Nam \& Tolman, 2002).

The participants were asked if they had experienced problems while in a relationship. More than half responded affirmatively. The most prevalent types of problems that affected a majority of those who reported relationship problems were communication (77.9\%) and jealousy or lack of trust (57.4\%). Other problems reported by at least $10 \%$ of the participants were family problems, financial problems, infidelity, and abuse. Notably, the incidence of abuse was lower than reported in most studies of IPV, an occurrence which will be discussed in light of the participants' perceptions of being victims or perpetrators of IPV. The relationship problems reported by the participants have important implications for the design of programs and interventions for preventing and dealing with IPV. 
To obtain a detailed and comprehensive picture of IPV as experienced and perceived by the group of Hispanic college students, this study combined close-ended and open-ended questions in addition to making use of several questionnaires that address the issue of different perspectives. A common limitation observed by Cuevas and colleagues (2010) in the existing research on IPV among Latinas has been focused on only one type of victimization. This study was based on the CDC (2006) definition of IPV, which encompasses actual or threatened physical or sexual violence or psychological/emotional abuse. Also included is the threat of physical or sexual violence used to control a person's actions. Thompson and colleagues (2005) also used this definition of IPV. WHO (2005) has employed a similarly comprehensive definition of IPV. In addition to encompassing different manifestations of IPV, the synthesis of quantitative and qualitative data provided insight into how the participants perceived different types of partner behavior as well as to how their perceptions matched objective indicators of IPV.

This study went beyond Cuevas and colleagues (2010) in that it examined victimization and perpetration of IPV by both genders. There has been some evidence from university students in the United States and Mexico that in couples where there is one physically violent partner, both partners have committed at least one act of violence (Straus \& Ramirez, 2007). While men are more likely to be perpetrators of sexual violence, women and men may be equally likely to be perpetrators and victims of psychological and physical abuse (Próspero, 2008; Wasserman, 2004). Attitudes toward violence and gender role orientation may have more influence on psychological and physical IPV than biological sex alone. 
Given the rigid gender role ideology inherent in Latin cultures, understanding gender role orientation and its relationship to IPV is essential to developing culturally relevant prevention and intervention programs as well as to working with and advising individual clients. At the same time, there is skepticism that Latinas living in the United States actually conform to traditional gender role norms (Ahrens et al., 2010). Even women with relatively low levels of acculturation are often eager to relinquish constraining gender role attitudes and embrace egalitarian ideals (Ramos-Lira et al., 1999). Young women and college students in particular, may be most inclined to abandon traditional gender roles. Stereotypical notions that Hispanic men endorse traditional gender roles and values can easily be counterproductive to efforts to prevent IPV.

This study was conducted from the perspective that an array of factors including acculturation, education and religious beliefs as well as individual differences in attitudes, beliefs, and experiences must all be considered in understanding IPV. The theoretical framework for this study was the theory of intergenerational transmission of violence based on Bandura's (1977) social learning theory. Observational learning, or modeling, is a cornerstone of social learning theory. From that perspective, witnessing domestic violence even without being victimized can have a pronounced impact on subsequent behavior. Estimates of the extent that the theory of intergenerational transmission of violence predicts future perpetration of abuse have varied tremendously, from $18 \%$ to $70 \%$ (Allen, 2001). Nonetheless, there is compelling evidence that it does indeed increase the risk of future domestic violence. WHO (2005) recognized family violence and prior victimization as significant risk factors for IPV victimization. 
It seems probable that the numerous variations reported from studies that have explored a link between childhood exposure to domestic violence and later IPV perpetration and victimization have to do with such factors as differences in how IPV is defined, methodological differences, and perhaps most important, the extent that the study examined the many other factors that influence relationship behavior. Thus, this study employed a number of research instruments, drawn from existing studies of IPV as well as studies of college students and Hispanic populations. In addition to a demographic survey, the participants completed the Conflict Tactics Scale-2 (CTS2), the Conflict Tactic Scale-Adult Recall (CTS2-CA), the Family Patriarchal Beliefs (FPB) scale, the Short Acculturation Scale for Hispanics (SASH), and the Extent of Religiosity Scale (ERS).

The CTS2 is probably the most widely used scale in studies investigating psychological and physical aggression and negotiation techniques in marital, cohabiting, and dating relationships. The CTS2-CA focuses on the participants' recall of parents' behavior toward one another. The juxtaposition of the two scales is ideally suited for exploring the relationship between childhood and adolescent exposure to family violence and young adult experiences and perceptions.

Neither quantitative nor qualitative research alone is capable of providing comprehensive understanding of an issue as complex as IPV. The survey instruments were all selected carefully, and the open-ended questions were designed to complement and enhance the data derived from survey measures. In all, the synthesis of quantitative and qualitative methods was selected as the optimum way of furthering understanding of how IPV is experienced and perceived among Hispanic young adult college students. 


\section{Discussion of Findings}

\section{IPV victimization and perpetration: Subjective perceptions versus CTS2}

scores. The existing research has shown there are numerous variations in the way IPV is conceptualized, operationalized, and perceived. At the individual level, personal factors such as those explored by this study may exert a powerful influence on people's subjective perceptions of IPV. Part of the rationale for combining quantitative and qualitative responses was the theory that there might be a marked discrepancy between the participants' subjective perceptions of IPV victimization or perpetration and the levels of victimization and perpetration as shown by their scores on the CTS2. Indeed, this was shown to be the case.

Findings of this study showed that very few of the participants saw themselves as victims or survivors or as perpetrators of IPV. Out of 120 young adult women and men, only $5 \%$ perceived themselves to be victims or survivors of IPV. The proportion that saw themselves as past or present perpetrators of IPV was even smaller, at $4.2 \%$. Although it is possible that unusual findings can be an artifact of a particular sample, even at superficial glance, such low figures stand in sharp contrast to the prevalence figures reported from prior studies of IPV regardless of population group.

For example, in their study of Mexican American college women, Coker and colleagues (2008) found that $43 \%$ had experienced some type of partner violence, including sexual violence (12.1\%), physical violence (19.7\%), threats of physical violence (11.5\%), battering (15.6\%), stalking (19.7\%), and psychological abuse $(30.2 \%)$. Especially troubling, and one of the reasons for conducting this study, was that only one quarter of the women who experienced physical or sexual violence considered it a 
problem in their relationship. Equally troubling was the high frequency of more than one type of violence.

Daley and Noland (2001) reported that two-thirds of the women in their diverse sample of community college students had endured verbal abuse by a dating partner within the last year. Ramirez (2007) reported a higher rate of IPV prevalence among Mexican American college students compared to White students (26\% versus 18\%). Although the difference between the two groups was not significant, it is obvious that the figures for either group are much higher than the $5 \%$ victimization and $4.2 \%$ perpetration perceived by the Hispanic South Florida students in this study.

Comparison between the students' subjective perceptions of IPV and their CTS2 responses works to illuminate the discrepancy between the students' perceptions of IPV victimization and perpetration and the findings from other studies, which are typically based on the CTS2 or a similar questionnaire. Out of the full sample of 120 students, 116 completed the CTS2. The participants' CTS2 subscale responses paint a completely different picture of IPV victimization and perpetration among this group. Roughly twothirds of the students acknowledged that they were victims or survivors or perpetrators of verbal aggression. The numbers were almost identical for both victimization and perpetration.

For sexual coercion, the figures approached one-third for victimization and perpetration according to CTS2 subscale scores. This finding was contrary to results reported from other studies including Cuevas and colleagues (2010), Flake and Forste (2006), Hazen and Soriano (2005), and Murdaugh and colleagues (2004), which all yielded higher rates of victimization among Latinas. Underreporting is a persistent issue 
in understanding the incidence and prevalence of domestic violence. According to Próspero (2008), effects for biological sex revealed that women were more likely to be perpetrators of psychological IPV, while men were more likely to perpetrate sexual IPV. Results from data obtained in this study indicated that both men and women were equally perpetrators of IPV. Thirty-one percent of the respondents reported being victims or survivors of sexual coercion, and $32.5 \%$ said they had used sexual coercion to perpetrate violence against their intimate partner. Physical assault was the least common type of violence, but the figures were nonetheless troubling. More than one-quarter of the participants (26.7\%) reported being victims or survivors of physical violence, and roughly $23 \%$ acknowledged physically assaulting a dating or relationship partner. Among those who had been victimized, 11.6\% reported that injury had occurred. Close to $16 \%$ of the perpetrators reported that there had been injury. Findings from the International Dating Violence Study showed that men and women were equally likely to be perpetrators of severe violence and to be chronic perpetrators of minor violence (Straus \& Ramirez, 2007). However, even when there is gender symmetry in the perpetration of physical violence, women are more likely to experience serious injury.

HCSs' perceptions of IPV. There was a sharp discrepancy between the participants' perceived experience as victims or perpetrators of IPV and their responses to questions related to partner aggression on a validated, quantitative assessment tool. The participants' responses to the question of what IPV means to them offer some insight into this phenomenon. There were relatively few references to verbal aggression, in contrast to the finding that two-thirds of the students had been victims and/or perpetrators of verbal aggression. In response to a question, HCSs agreed that use of force, coercion and 
intrusion to gain power and control are actions that define IPV. One participant included both physical and verbal aggression, stating, "Partner violence to me includes physical violence when someone is hurt or verbal abuse when a partner is constantly belittled." However, this definition seems to imply that a certain level or frequency of aggression must be reached in order to qualify as IPV.

There were several references to sexual abuse and coercion in the participants' descriptions of what IPV means to them. Most responses under the open-ended question "What does intimate partner violence mean to you?" may suggest normalizing acts of violence which could account for the discrepancy between the very low levels of perceived victimization and perpetration and the fairly high levels of sexual coercion (i.e., victimization and perpetration) shown in CTS2 scores. Normalizing violence was dominated by responses related to sexual behavior. One participant explicitly commented, "Thrill seeking and limit testing: Sex versus passion.” While these responses may refer to behavior in which people freely engage, they are disturbing in light of the prevalence of sexual coercion based on findings from the CTS2.

References to the use of physical violence and sexual force to gain power and control were more direct. There is no denying that "Beating you, forcing them [sic] to have sex," is a prime example of coercion and intrusion to gain power and control, which constitutes a form of violence. It is not surprising that more overt forms of violence, including constant reliance on verbal abuse to denigrate a partner, are recognized as manifestations of IPV. However, more subtle forms of abuse can have insidious consequences simply because they are easier to deny or ignore. 
One interesting response was that one participant suggested that she or he identified with the perpetrator's abusive behavior. This was intriguing and warrants greater attention. One participant exemplified this phenomenon by first presenting her or his definition of IPV and then describing its personal consequences. To this participant, "Mentally, physical and emotional pain is [sic] considered intimate partner violence." This participant had been unable to escape from a mentally and emotionally abusive relationship for more than a year. Although the relationship finally ended, the person related that, "When I did get out of it, I become very defensive, and unfortunately, I do verbal abuse which I'm trying to change."

It is not unusual for abusive relationships to be composed of two abusive partners. However, this particular side of IPV, namely that the abused becomes the abuser is often neglected in research, perhaps because of excessive reliance on quantitative data and analyses. Independently and collectively, the concepts that arose from the descriptive responses to the meaning of IPV offer insight into the phenomenon of IPV that an instrument such as the CTS2 cannot provide. When these responses are analyzed in conjunction with the sharp disparity between the participants' subjective perceptions of their experience of IPV victimization and perpetration and their responses on the CTS2, it highlights the complexity of IPV and the importance of recognizing the unique ways in which IPV is perceived in order to address the problem at the individual and group level.

Student participants' perceptions of IPV versus parental perceptions. The participants in the open ended questions were asked what they thought IPV meant to their parents. This question produced four concepts which suggest that aggression and intimidation, learned and observed behaviors, generational disconnect, and normalizing 
acts of violence with an intimate partner exist in the minds of the HCSs as their parents' meanings of IPV.

Notably, under the concept of aggression and intimidation, the primary focus was physical violence with one exemplar, "A father beats a mother." The issue of domestic violence first came to light in the context of male batterers and female victims. Johnson (1995) coined the term patriarchal terrorism to denote physical and psychological violence performed by men for the explicit purpose of controlling their female partners. Johnson recently renamed patriarchal terrorism intimate terrorism in response to evidence showing that women could also engage in a high degree of controlling behavior combined with physical aggression (Graham-Kevan \& Archer, 2008). In Hispanic families, adherence to the cultural gender roles prescribed by machismo and marianismo make it more likely that men are the perpetrators of physical violence, and women may feel they have to endure an abusive relationship (Kasturirangan \& Williams, 2003; Vandello et al., 2009). Two responses included verbal and mental abuse. One exemplar was "To my parents, violence is something that damages the partner either physically or mentally." Another described aggression and intimidation as "Slapping one another in the face; yelling profanities at each other." For example, one participant considered "throwing shoes at each other" part of a "typical Cuban household." This response raises the prospect that young adults of a particular cultural group may view certain aspects of IPV as "normal" in their own group although they are aware that it may well be viewed differently (and negatively) within the greater society. Ramos Lira and colleagues (1999) prefaced their study by noting that interpretations of the terms rape and sexual violence 
vary considerably from one culture to another. Acts that are criminalized in some cultures can be ignored or even condoned in others.

Findings also suggest that HCSs believe that their definitions of IPV were most likely parallel to their parents' perceptions of IPV. This was exemplified by overwhelming response of "Same as with my parents." This concept is directly related to the theory of intergenerational transmission of violence, which served as a framework for this study. Building on the generational theme, the participants were asked whether they believed their own definition of IPV was similar to those of their parents. Reasons for sharing similar perspectives included talking with parents "about everything" and sharing "most of the same views," feeling the common viewpoint "makes sense and is logical" and feeling "it's the same to me no matter what."

The response of one participant under this concept was consistent with the changes in conceptions of IPV that have taken place since the problem of domestic violence first gained attention in the 1970s: "I believe it is similar, but I have a broader range of what partner violence is while my parents' viewpoint may only be isolated to physical violence." At the same time, the disparity between the students' perceptions of violence and their CTS2 subscale scores suggests that many may still have a limited viewpoint on what constitutes IPV even if it is not restricted only to physical violence. In particular, the participants seemed less aware of the potentially damaging impact of psychological abuse (WHO, 2005).

The response "Rough sex is good," which suggests normalizing violence, is a particularly troubling trend among a high proportion of college students who regard some degree of violence in dating relationships as "acceptable or normal in some circumstances 
[original emphasis]" (Wasserman, 2004). The participant who made this comment attributed this sexual preference to growing up in a household with parents who enjoyed violent sex. Once again, the troubling aspect of this comment is the question of whether it refers specifically to consensual sex or whether the person may attempt to impose violent sex on partners on the rationale that others should find it enjoyable. Interestingly, the concept of normalizing violence produced another exemplar in which the participant commented "I believe some people enjoy physical abuse when intimate, and I feel my parents won't agree with that." This perception contrasts with the participant whose parents transmitted the idea that violent sex was good. It is important to recognize that there are numerous individual differences in perceptions of partner violence of any type by people who share the same cultural heritage (Vandello \& Cohen, 2003).

Another concept that emerged indicated a form of generational disconnects which is embodied by a participant who declared, "I honestly don't think my parents have a clue," and could think of no example. The parents were described as "Gen X extreme Old School, uneducated." Rather than a generational difference per se, it may be the difference in educational level between the uneducated parents and the college educated young adults that creates the main disconnect in conceptions of IPV between the two generations.

Types of interpersonal violence identified by HCSs. When asked to describe different forms of violence, the participants recognized that the notion of violence extends beyond physical force. The five types of violence cited were physical, emotional inflicted by verbal aggression, psychological/mental, social, and sexual. There was a substantial degree of overlap in the exemplar statements. Verbal aggression included any 
type of abuse, "violation of personal decisions or right to act," and "Yelling at your partner to make your partner something of no value, inferior to you." These last two statements exemplify the ways that psychological abuse is used as a means of control (McCloskey et al., 2007; Nam \& Tolman, 2002). Often, it is used in conjunction with physical violence, which was explicit in the exemplar statement under physical abuse and assault: "Aggressive, physical, emotional, mental, verbal."

In parallel fashion, physical violence was included with emotional and verbal aggression in the psychological and mental typology, which broadly encompassed "anything that includes taunting, belittling, and messing with the emotions or physical pain that is subjected onto someone else." Emotional abuse included making the other person feel guilty as a means of controlling their actions and getting them to do what the abusive partner wants, along with verbal abuse such as name-calling, insulting, and swearing. The social exemplar included "different types of violence such as physical, mental, verbal abuse." Notably, bullying also fell under the social category, with the comment "Bullying has also become very popular in cyberspace." Former relationship partners are frequent targets of electronic bullying (Belsey, 2008). Although the problem is beyond the scope of this study, it is an extremely important topic for future research.

Overall, the participants' qualitative responses showed that they were aware of different types of intimate partner violence as well as aware of similarities and differences between their own and their parents' perceptions of IPV. Ironically, they appear to be less aware of their own experiences as victims and/or perpetrators of IPV.

Cultural gender roles, parental IPV, acculturation, and religiosity. Research questions two through six all addressed the interactions of cultural gender roles, adult 
recall of parental IPV, acculturation, and religiosity, with differing emphases on victimization and perpetration and the relative contributions of each of the factors examined.

Cultural gender roles. The degree of acceptance of cultural gender roles was examined by the Familial Patriarchal Belief scale. There was minimal evidence of patriarchal beliefs in this sample of Hispanic young adult college students. The overwhelming majority of the students $(>86 \%)$ disagreed with the idea that a man has the right to decide whether his wife, partner, or girlfriend should work outside the home or go out in the evening with friends, and more than $90 \%$ expressed disagreement with the idea that a man has the right to have sex with his wife, partner, or girlfriend when he wants to even though she may not want to. For each of these questions the predominant response was strong disagreement.

The findings of this study showed gender differences in cultural gender roles. Although the number of female participants was almost twice the number of male participants in this study, based on their mean rank scores, male participants had higher cultural gender role mean rank score than female participants. Male participants' mean difference scores ranged from .47 thru .90 on almost all of the FPB scale items (with predominant response of strong disagreement) with the exception to an item on protection by law for beatings. Female participants endorsed protection more than did male participants.

There was somewhat less disagreement with the idea that sometimes it is important for a man to show his wife, partner, or girlfriend that he is head of the house, though a majority disagreed and strongly disagreed. More than $20 \%$ of the students 
agreed with the belief that sometimes it is important for a man to show he is the head of house, while $15 \%$ were undecided which suggests that to some degree, these students accepted certain aspects of machismo.

An unexpected and somewhat alarming finding was that more than $10 \%$ of the participants disagreed with the statement that women should be protected by law if their partners beat them, including 10 respondents who disagreed strongly. Physical assault is an illegal behavior in the United States. However, people who come from "honor cultures" may be more inclined to accept physical violence if it is in the service of preserving a man's honor (such as in response to an act of infidelity). Vandello and Cohen (2003) found this to be the case in their study of college students from Brazil and from the southern United States. However, the U.S. students expressed a more negative view of a man who hit his wife than their Brazilian counterparts.

The predominance of women in this study may have been a reason why there was such limited acceptance of traditional gender roles. At the same time, the women alone could not have accounted for the very high proportion of participants who expressed egalitarian gender attitudes. The young age and high educational level as well as the diverse cultural environments of south Florida where the students reside are all factors that could easily counteract acceptance of rigid prescribed gender roles by women and men. In their study of Hispanic college students, Raffaelli and Ontai (2004) found that the male and female students experienced very different socialization growing up. All the women said they had more restrictions on their activities than their male peers. Similarly, the men reported that they had more freedom at home than their sisters or other female relatives. The experience of being restricted at home could easily make women more 
eager to embrace egalitarian attitudes. However, men who grow up in homes with traditional gender roles may be reluctant to relinquish male privilege. Men are more likely to express positive attitudes toward machismo than women (Ruiz-Balsara, 2002).

Experience of parental IPV. In terms of the participants' recall of parental IPV, the negotiation subscale produced the highest means, followed by verbal aggression. In light of these results, it is possible that the high level of witnessed parental negotiation may have accounted for the belief that Latina women should cater to men and be submissive and self-sacrificing (Kasturirangan \& Williams, 2003). Thus, it is not surprising to conclude that the acceptance of traditional gender roles leads to the acceptance of domestic violence and the willingness of abused Latinas to remain in abusive relationships (Kasturirangan \& Williams, 2003; Vandello et al., 2009). Injury between partners produced the lowest mean. It is not surprising given these findings that there were numerous references to verbal aggression in the participants' qualitative descriptions of IPV. Próspero (2008) noted that there has been empirical evidence that supports a social learning theory of interpersonal violence, specifically citing research that showed that adolescents are more likely to perpetrate dating violence if they witnessed domestic violence at home, if they were witnesses to dating violence, or their peers were involved in antisocial behavior.

Acculturation. Findings indicated that there is no difference in the level of acculturation between genders. The participants' responses showed that English was the preferred language of a majority of participants. While English as a second language (ESL), bilingualism, or English immersion classes would have been pivotal to the shift from Spanish to English or bilingualism, it is probable that attitudes toward the values 
and beliefs of the dominant Anglo culture would also play a role in the choice of language. In terms of social group preference, close to half the students reported that their chosen friends consisted of more Latinos than Americans or were all Latinos; however, almost one-third reported having roughly equal numbers of close friends from both cultural groups, and over one-fifth reported that more or all of their close friends were American. Ramirez (2007) acknowledged that the English-speaking, relatively highincome Mexican American university students comprised a sophisticated group with minimal differences in acculturation between genders. Social integration, however, did affect the prevalence of IPV despite the fairly high levels of social integration found for the Mexican American and White students as a group. Specifically, higher levels of social integration were linked with lower prevalence of IPV, thus supporting the socialcontrol theory of interpersonal violence.

Incidentally, in this study, HCSs' perceptions of their level of victimization and perpetration greatly contrasted with their CTS2 scores which further increased their risk of becoming chronic perpetrators and/or victims of IPV. Harris and colleagues (2005) also explored acculturation in IPV, and results showed that the greater degree that the women adhered to traditional gender roles, the less likely they were to report incidents of IPV. According to Harris and colleagues (2005), their findings suggested that Mexican American women with very traditional gender role attitudes may not recognize a partner's behavior as abuse. The researchers found it striking that gender role beliefs had such a powerful role on reporting for both the Mexican-born and U.S.-born respondents, transcending sociodemographic characteristics and family dynamics that distinguished the two groups. Likewise, among the domestic violence survivors interviewed by 
Kasturirangan and Williams (2003), marianismo was found to be a key factor in reluctance to report abuse.

Religiosity. The HCSs' responses on the Extent of Religiosity Scale contradicted the assumption that Hispanic people are very religious. This study illustrated the importance of eschewing stereotypical generalizations in characterizing members of different cultural groups as the largest proportion of participants (almost half of the surveyed participants) described themselves as somewhat religious and roughly one-third described themselves as either not religious or not at all religious. Only slightly over onefifth of the participants considered themselves religious or very religious. Higginbotham and colleagues (2007) explored levels of religiosity among 18- to 24 year-old females wherein participants who reported experiencing low religiosity also reported more courtship violence (victimization) compared to those who have high-religiosity experiences. On the contrary, this dissertation did not indicate that religiosity is associated nor a predicting factor on participants' levels of victimization and perpetration as well as on their perceived IPV victimization and perpetration.

\section{Relationships among gender, cultural gender roles, parental IPV,} acculturation, religiosity, and IPV victimization. Analyses revealed a significant but weak association between being a victim or survivor of IPV and adult recall of parental IPV. A moderate to low correlation emerged between victimization and parental recall of verbal aggression. Researchers who have investigated domestic violence in the United Kingdom reported that $90 \%$ of the children are in the same or the next room when the violence occurs (Leason, 2005). This may account for the significant level of parental recall of verbal aggression as reported by the HCSs in this study. No significant 
differences were found between male and female participants related to level of IPV victimization. Overall, gender, traditional gender role attitudes, acculturation, and religiosity were not significantly linked with level of victimization by a dating or relationship partner.

\section{Relationships among gender, cultural gender roles, parental IPV,} acculturation, religiosity, and IPV perpetration. A significant but weak relationship was found between the acculturation and perpetration of IPV. These results supported the findings of Hazen and Soriano (2005) and Lown and Vega (2001) who found that women who were more acculturated were more likely to have experienced IPV. The relationship between acculturation and IPV was due in part to higher prevalence of IPV among Latinas born in the United States.

A moderate to low association emerged between exposure to verbal aggression by parents while growing up and perpetration of IPV. Exposure even to one act of violence can make children hypersensitive to any display of aggression or conflict, including verbal aggression is which extremely common in families. There is evidence that continued exposure to psychological aggression may be even more damaging to children's psychosocial development (Panuzio, Taft, Black, Koenen, \& Murphy, 2007).

No significant difference between male and female participants was found related to level of perpetration of IPV. There were no significant relationships between traditional gender role attitudes, religiosity, and the participants' recall of parental IPV.

Relative contributions of gender, cultural gender roles, parental IPV, acculturation, and religiosity to IPV victimization. Multiple linear regression analysis was used to test for the relative contributions of major study variables and participants' 
level of IPV victimization. The results showed recall of parental IPV to be a significant predictor of IPV victimization. On the assumption that all factors remain constant, for each unit increase in CTS2-CA scores, the person's level of victimization would be expected to increase by 0.4 . Further regression analysis excluding the non-significant variables confirmed the significance of parental IPV as a factor in IPV victimization.

These findings highly supported the theory of IGTV. Observational learning (modeling) is a cornerstone of social learning theory; thus, witnessing domestic violence even without being victimized can have a marked impact on later behavior. A number of factors affect how children interpret family violence and its impact on their subsequent tendency toward being a perpetrator or victim of violence. These include age, gender, temperament, the nature of the aggressive acts, the children's relationships with their parents, the prevalence of community violence, and cultural attitudes and beliefs (Fosco, DeBoard, \& Grych, 2007). Of these factors, the least is known about how culture shapes children's attitudes toward interpersonal violence. Men who are violent toward their partners are prone to be violent toward their children as well. Abused women often turn the anger and aggression they dare not express to their partners to their children. Regardless of whether the children are physically victimized, the chronic stress endured by abused women compromises their parenting skills and has a direct impact on the children's psychological health (Huth-Bocks \& Hughes, 2008).

\section{Relative contributions of gender, cultural gender roles, parental IPV,} acculturation, and religiosity to IPV perpetration. As with Hypothesis 4 addressing the relative contribution of these variables to IPV victimization, multiple linear regression analysis was used to test the relative contributions of the major study variables to the 
participants' level of IPV perpetration. In contrast to the findings for victimization, none of these variables emerged as a significant predictor of level of IPV perpetration.

Gender symmetry in IPV. The issue of gender symmetry in victimization and perpetration of IPV is controversial. According to CDC (2011), each year, women experience about 4.8 million intimate partner-related physical assaults and rapes. Men are the victims of about 2.9 million intimate partner-related physical assaults. Domestic violence was first brought to public attention by feminists, and feminist theory and claims that IPV arises from patriarchal social structures that socialize males and females into rigid, hierarchical gender-specific roles (Próspero, 2008). Violence is seen as stemming from men's exercise of power to control women. From this standpoint, the emphasis is on "the patriarchal family," the social construction of masculinity, and the structural factors that restrict a woman's ability to break away from IPV victimization" (p. 640). According to the feminist perspective, men represent the vast majority of perpetrators of IPV and women the vast majority of victims.

However, even Johnson (1995), who elaborated the concept of patriarchal terrorism has since replaced it with intimate terrorism in recognition of evidence that women are capable of exhibiting comparable levels of controlling and physically aggressive behavior (Graham-Kevan \& Archer, 2008). Throughout society, the traditional stratified gender roles that made male violence acceptable and constrain women's ability to escape from abusive relationships are rapidly disappearing. At the same time, there seems to be more acceptance of some degree of physical violence in dating relationships, especially among college students (Wasserman, 2004). The 
qualitative responses of the participants suggested that verbal aggression, unless it is ongoing and/or overtly belittling, may not be considered relationship violence.

The analyses showed no significant differences between male and female participants in the experience of either IPV victimization or perpetration. The finding of absence of significant gender differences for victimization or perpetration of physical or psychological aggression is consistent with findings from other studies (Próspero, 2008; Wasserman, 2004). Overall, men and women shared many similar risk factors for both perpetration and victimization of IPV (Cummings, Gonzalez-Guarda \& Sandoval, 2013). One gender difference is that women are more likely to sustain serious injury from physical violence (Strauss \& Ramirez, 2007). However, reports of physical injury were low among the participants in this study.

Latinas who endure abusive relationships typically do so more out of fear and lack of access to resources that would allow them to leave than to adherence to cultural constructs of machismo and marianismo (Edelson et al., 2007; Gonzalez-Guarda et al., 2009; Montalvo-Liendo et al., 2009; Murdaugh et al., 2004; Rodriguez et al., 2001). Additionally, Latina survivors of domestic abuse have themselves commented that women who are more educated and independent are less apt to accept the belief that women should cater to men and be submissive and self-sacrificing (Kasturirangan \& Williams, 2003). Clearly, the vast majority of women and men in this study rejected that attitude. The convergence of factors including the participants' young age and educational level, rejection of traditional gender role attitudes, relatively high acculturation, and low religiosity all pointed toward a group in which there would be relative gender symmetry in IPV victimization and perpetration. 
Cultural gender role differences in IPV. Based on this dissertation, the author surmised that there were at least five factors that influenced traditional Latin gender role ideology among HCSs, namely: marianismo, machismo, familismo, respeto and honor culture. Although the push toward traditional gender roles and curtailment of personal freedom intensified during adolescence, acceptance of traditional cultural gender role was evident in this study. Despite an over-whelming rejection of cultural gender role ideology among HCS participants, there was a marked significant difference between genders and cultural gender role acceptance. Female HCSs reported lower average mean rank score as compared to male participants. In this study, female participants reported that they were more likely to embrace patriarchal gender role ideology as compared to their male counterparts.

Personal attitudes toward traditional gender scripts vary tremendously among individuals within the same cultural group (Vandello \& Cohen, 2003). Understanding gender role socialization among the Hispanic/Latin college students may provide insights as to how and why mothers' treatment for girls and boys differs. An example was a study by Raffaelli \& Ontai (2004) wherein parents' enforcement of stereotypically feminine behavior for daughters, and the restriction of girls' activities outside of the home; and for sons, a mother's traditional gender role attitudes resulted in encouragement to engage in traditionally masculine behavior. For daughters, traditional gender role attitudes on the part of mothers and fathers translated into encouragement to adopt stereotypically feminine behavior. A father's egalitarian attitudes and use of English at home had some influence on the son's encouragement to do household chores (Raffaelli and Ontai, 2004). 
The parents' own gender role attitudes were the predominant factor in Hispanic/Latin gender socialization practices (Raffaelli and Ontai, 2004). It is not surprising that based on statistical analyses in this dissertation, patriarchal beliefs were directly related to perceived perpetration of IPV. Adult-recall of parental IPV was closely associated with perceived perpetration and a significant predictor on experiences of IPV victimization. A strong relationship was noted between cultural gender role and perceived perpetration, suggesting congruencies with the theoretical framework of IGTV.

Although gender role attitudes may explain in part gender differences among HCSs participants' gender role mean scores, one must be mindful that factors contributing to this phenomenon may be more difficult to explain. Lack of contextual meanings as prime limitations of self-report surveys and measures further hinders full understanding on how gender role attitudes and differences can be operationalized. Jakupcak, Lisak, and Roemer (2002) surmised that high levels of gender role stress could provoke a violent response, particularly in conjunction with a high degree of masculine gender role ideology. This "chivalrous" aspect of traditional masculinity is sometimes conceptualized as positive machismo whereas controlling behavior and violence represent negative machismo. Devotion and dedication to his children and to the women in his family are attributes of machismo, along with courage, strength, and indomitable will (Snyder et al., 2010). However, the allegedly positive chivalrous side of machismo carries negative implications for women because it implies that women are weak, vulnerable, and in need of protection (Bracero, 1998). 


\section{Intergenerational Transmission of Violence}

Findings revealed a significant albeit weak relationship between IPV

victimization and adult recall of parental IPV and a moderate to low relationship between adult recall of verbal aggression by parents and perpetration of IPV. When multiple regression analysis was used to examine the relative contributions of each of the variables under study, adult recall of parental IPV was significantly linked with the level of victimization but had no significant effect on the level of IPV perpetration.

There is some dispute over the extent that the theory of intergenerational transmission of violence predicts violent behavior in adulthood, with estimates ranging widely from $18 \%$ to $70 \%$ (Allen, 2001). However, even the lowest estimates have supported the premise that being exposed to domestic violence in childhood, even as a witness, increases the risk for future perpetration of domestic violence. Turning to researchers Kaufman and Ziegler $(1987,1993)$, Allen cited an intergenerational transmission rate of $30 \%$ as a "best estimate," noting that a figure of $30 \%$ is six times the base rate of abuse in the general population" (p. 63).

WHO (2005) recognized family violence and previous victimization as prominent risk factors for IPV victimization. However, there are typically many interacting factors involved. Hence, this study examined several variables that have been found to play a role in IPV victimization and perpetration. The findings supported a weak but significant association between the participants' recall of parental IPV and IPV victimization. Furthermore, exposure to parental IPV predicted increases in the level of IPV victimization. No parallel effect was observed for a link between parental IPV and IPV perpetration among HCS. There was some association between the participants' recall of 
verbal aggression by their parents and perpetration of IPV. This association was further supported by the qualitative descriptions of IPV, which contained a number of references to verbal aggression. The students' scores on the CTS2-CA showed limited evidence of exposure to physical violence in childhood compared to exposure to parents' verbal aggression. Overall, the findings supported a limited but still significant effect of childhood exposure to parental aggression and IPV in young adulthood among the Hispanic college students who participated in this study.

\section{Implications for Future Research}

Despite the burgeoning population of individuals of Latin heritage in American society, including their increasing presence on college campuses, there has been very limited research on IPV in this population group. Several researchers who investigated IPV among women and men of Latin heritage deliberately focused on college students due to the high incidence of IPV among young adults (Coker et al., 2008; B. A. M. Smith et al., 2006; Ramirez, 2007). The problem of dating violence on college campuses assures that the issue of IPV among college students should be a focus of additional study.

A mixed methods approach that combined qualitative and quantitative methods was deemed the best way to gain insight into the phenomenon of IPV victimization and perpetration among Hispanic college students. There was a marked discrepancy between the participants' perceived experiences of IPV as victims or perpetrators and their experiences of verbal aggression and sexual aggression based on quantitative CTS2 scores. Five percent of the participants considered themselves victims or perpetrators of IPV, yet roughly two-thirds would be classified as victims or perpetrators of verbal 
aggression on the CTS2, and roughly one-third emerged as victims or perpetrators of sexual coercion. Further qualitative research is warranted to illuminate why there is such as sharp discrepancy between Hispanic college students' subjective perceptions of IPV and their responses on a standard instrument such as the CTS2.

Qualitative research often serves as a precursor to quantitative research. A qualitative exploration of Hispanic college students' perceptions of IPV could be used to create a questionnaire for future quantitative study. Most research on IPV among Latinos is conducted with Mexican Americans, who comprise the largest proportion of Latinos in the United States. In this study, one participant made specific reference to IPV as characteristic of a "typical Cuban family." While that statement is an obvious overgeneralization, there is greater danger of making stereotypical generalizations if researchers do not acknowledge the heterogeneity among the many national groups that are classified as "Hispanic" or "Latino." More research is needed to explore how IPV is perceived and experienced among young adults descended from different Latin countries.

The participants in this study were educated, relatively acculturated, and reported to have low religiosity level. Thus, it was not surprising that for the most part there was overwhelming rejection of traditional gender role ideology. At the same time, close to one-third of the participants expressed support for or were neutral regarding the idea that sometimes it is important for a man to show his wife, partner, or girlfriend that he is head of the house. This finding suggested that even among Hispanic college students who embrace largely egalitarian gender role attitudes; there is some residual support for machismo. Greater understanding of how acculturated college students view machismo 
may be important for designing programs and interventions to prevent and address IPV among HCS.

Given the complexity of the problem, interdisciplinary research such as collaborative investigation of IPV among various disciplines, is integral in assessing and evaluating IPV in a multi-dimensional aspect. Social scientists who are experts in diverse fields of research including nursing, medicine, social work, psychology, and criminal justice may have differing points-of-views as to how and why IPV persists in society at large. In addition, the author of this dissertation aims that this study serve as a foundational program of research in exploring youth and family violence in the community, the development of evidence-based tools, evaluation, and research outcomes sensitive to specific cultural groups such as:

1. Exploring early life protective factors that buffer or cushion youths from the effects of family violence.

2. Find ways to enhance these protective aspects.

3. Development of predictive assessment tools that may be used to project possible involvement on self-directed violence such as suicide; interpersonal violence such as bullying, intimidation and relationship violence; and collective violence such as gang rape / aggression.

4. Exploring "college student stress" and resilience that may contribute or curve acts of aggressive/violent behaviors.

5. Further exploring youths' attitudes towards IPV and whether certain attitudes can predict acceptance and/or rejection of violence.

6. Expanding on the discovery of symmetry on gender violence. 
7. Exploring the role of sexual orientation [lesbian, gay, bi-sexual and transsexual] on IPV and dating attitudes.

8. Creating collaborative projects with research academic institutions such as Florida International University's (FIU) schools of Medicine, Psychology, Social Work and Public Health by creating a community-based opportunities for primary and secondary prevention of IPV (i.e., wrap-around services such as counseling, development of positive coping skills, etc.) and demystifying family violence among Hispanic youths and families.

9. Creating partnerships with local elementary, middle and high schools in promoting campus-wide awareness of IPV.

\section{Implications for Nursing and Interdisciplinary Practice}

Young adult college students are at a stage where they are beginning to form intimate relationships. The findings from this study help confirm that exposure to parental violence may influence IPV victimization, and perhaps to a lesser degree, perpetration in young adulthood. There is evidence that the incidence of IPV increases

during youth and young adulthood (Noonan \& Charles, 2009). College students may find themselves in an environment in which some aspects of IPV are considered normal behavior. These realities heighten the challenge of preventing violence in dating and intimate partner relationships.

More than half of the participants in this study acknowledged that they had some type of relationship problems. More than three-quarters reported problems with communication, and more than half reported problems with jealousy and lack of trust. These two areas, communication in particular, offer a focus for targeting interventions. 
In fact, the CTS2 is based on theory that positive communication tactics are key to preventing partner aggression.

In a study that explored what Hispanic men and women sought in a marriage education program, domestic violence and conflict resolution skills were cited as important topics by both men and women (Snyder et al., 2010). It is also notable that men and women both espoused fluid gender roles. College students' perceptions of IPV can be an important springboard for designing interventions that secure their interest and have the potential to change negative attitudes and behavior. The desire for learning conflict resolution skills may be a reflection of the communication problems that were prevalent among the participants in this study. Conflict resolution skills and more broadly, communication skills, are valuable for college students to use in any type of interpersonal relationships. Health and social service providers must be cognizant that contributing factors to either victimization and/or perpetration of IPV among college students must be addressed first (i.e., perceptions of IPV, socioeconomic, cultural factors, etc.). This is vital for any type of IPV intervention program. Failure to do so may limit intervention outcomes, or interventions may not be successful at all.

There are various support and intervention programs in colleges and universities in the United States. The Victim Advocacy Center at Florida International University is a prime example of a comprehensive counseling and psychological services for victims of IPV. The center has extensive online resources as well as on ground resources, personal counselors, and IPV advocates that would assist college/university students. Its mission statement is: 
Provide confidential assistance to FIU students, faculty, staff, and university visitors who have been victimized through threatened or actual violence and to support the healing process. Traditional college-aged students are an at-risk population for violence, and staff are dedicated to assisting students remain successful in their academic pursuits. In addition, the Center seeks to enhance safety and promote healthy relationships by sponsoring awareness activities, prevention education, peer education, and collaboration with university officials. Through clinical practice and research, the Victim Advocacy Center aims to contribute to the body of knowledge and influence public policy regarding to issues related to victimization. (Florida International University, n.d.) The Center of Excellence for Health Disparities Research at Miami University (Miami, FL) is a comprehensive research initiative funded by the National Institutes of Health, National Institute on Minority Health and Health Disparities (NIMHD). "El Centro develops tests and disseminates culturally tailored interventions to improve the health of groups who are disproportionately affected by HIV/AIDS and other sexually transmitted infections, drug abuse, intimate partner and family violence, and co-occurring mental and physical disorders" (University of Miami School of Nursing and Health Studies, n.d.).

Another college/university outreach program in relation to IPV is the Intimate Partner Violence Assistance Clinic (IPVAC). IPVAC is a multidisciplinary clinic at the Levin College of Law which provides indigent victims of domestic as well as dating and sexual violence with legal representation, mental health counseling, and case management needs. 
Various federal agencies such as the U.S. Department of Justice (USDOJ) have created grant programs under the Office on Violence Against Women (OVW) which funds 21 programs such as the Campus Grant Program which encourages institutions of higher education to adopt comprehensive, coordinated responses to domestic violence, dating violence, sexual assault, and stalking (www.ovw.usdoj.gov). Other programs include Children and Youth Exposed to Violence Grant Program which "seek to mitigate the effects of domestic violence, dating violence, sexual assault, and stalking on children and youth exposed to violence and reduce the risk of future victimization or perpetration of domestic violence, dating violence, sexual assault, and stalking" and Services, Training, Education and Policies to Reduce Domestic Violence, Dating Violence, Sexual Assault and Stalking in Secondary Schools Grant Program (STEP) which is a "discretionary grant program is designed to support projects that provide training to school administrators, faculty, and staff; develop policies and procedures for response; provide support services; develop effective prevention strategies; and collaborate with mentoring organizations to support middle and high school students who have experienced or are victims of domestic violence, dating violence, sexual assault, or stalking" (www.ovw.usdoj.gov, n.d.).

Policy makers in Florida passed prevention initiatives which include early education about healthy dating practices. The 2010 Florida Laws, Chap. 217 (2010 SB 642/HB 467)

Requires a comprehensive health education taught in the public schools to include a component on teen dating violence and abuse for students in grades 7 through 12. Would require district school boards to adopt and implement a dating violence 
and abuse policy and provides policy requirements. Also would require the Department of Education to develop a model policy that includes school personnel training. (http://www.ncsl.org, n.d.)

The CDC (2011) continues to focus on reducing the factors that put people at risk for victimization while increasing the factors that protect people from becoming perpetrators of violence. Extensive research has been conducted in various settings that have emphasized developing and evaluating prevention strategies throughout the lifespan. With a focus on college students, barriers must be addressed. Issues included are (a) college students feel trapped by the social networks and/or the closed environment of many campuses; and (b) students may feel isolated from their personal support networks and resources for help as these students may be away from home for the first time. This is especially true if the student is also from a different state or country (Break the Cycle, Inc. 2005). Additionally, students may have a small or limited social network due to the college campus atmosphere, cannot afford supportive services, or cannot even seek available resources. Some students may not define their experience as abusive, as found in this study.

Nurses play a pivotal role in college and university campuses. These roles not only comprise of acute clinical practice in college health rooms and clinics, but also include teaching and demonstrating healthcare actions to college students and the community by which the institution serves. Such topics may include health promotion and wellness; injury, illness and disease prevention based on the primary level of community health intervention. Nurses are also seen as community leaders, advocates and scholars. For nurses who work with individual clients, it is very important to 
recognize that there are numerous individual differences in attitudes toward IPV among individuals from the same cultural group (Vandello \& Cohen, 2003). The findings of this study also illustrate that it is misguided to assume that Hispanic college students adhere to traditional prescribed gender roles or are guided by strong religious beliefs. For any efforts to address the sensitive issue of IPV to be successful, it is essential to gain insight into how IPV is perceived and experienced at the individual and group levels.

Lutenbacher, Cohen, and Mitzel (2003) conducted a focus group study of White and African American women who were primarily high school graduates employed in low earning jobs. Four themes arose from the study. The first was Living and Unnatural Experience, which detailed physical and emotional responses to violence, including diminished self-esteem and chronic health problems; compartmentalizing their private and public lives; concern over their children's ongoing exposure to violent and dangerous situations; and unawareness of available resources as well as uncertainty of what would happen if they sought help. Second was The Experience of Telling, which covered obstacles to disclosure, insensitivity on the part of nurses and doctors, and professionals' lack of understanding of why they stayed in the abusive relationship. Third was The Leaving Experience, which ranged from impulsive to carefully and strategically planned. Lack of financial resources severely constrained them from leaving. Most could not afford lawyers and perceived inequities in the court system. They lacked the education and job skills for financial autonomy and had no secure housing options. This led to the act of returning, which often recurred several times due to "inadequacies of the helping systems, the lack of resources, their own ambivalent feelings, and their desire to keep their families intact" (p. 61). 
The final theme was Reducing Barriers. Paralleling the women interviewed by Bent-Goodley (2004), their overriding recommendation was educating the larger community and "working with young children to break the cycle of abuse and reduce the tolerance of violence in society" (Lutenbacher et al., 2003, p. 61). These types of interventions are now widely available in various educational institutions, universities and colleges. They also advocated through educating professionals about domestic violence including emphasis on regular screening for abuse in health care facilities with onsite intervention, which many professionals recommend (Tower, 2003, 2006). Their additional recommendations were making services more accessible and available, particularly family-oriented services as well as survivor advocacy, emanating from a desire to help other women.

Noting that there has been no cohesive framework for guiding a study on advocates' risk assessment techniques, A. R. Roberts (2007) devised a typology of "woman battering" to guide prevention and intervention efforts by clinical and forensic professionals. While a better title might be warranted, the classification system encompasses a full spectrum of abusive relationships and offers useful guidelines for helping professionals understand the dynamics of relationships characterized by IPV.

\section{Risk Assessment}

A. R. Roberts' (2007) strategy for working with battered women is based on crisis intervention targeted to the specific level of abuse. The model has seven stages: (a) assessing danger and lethality, (b) building rapport and communication, (c) identifying and prioritizing the most important problems, (d) dealing with feelings and providing support, (e) exploring potential alternatives, (f) devising an action place; and (g) follow- 
up actions. Roberts (2007) described predicting the duration and severity of IPV as "among the most complex issues in forensic mental health and psychological risk assessment" (p. 526). The complexity is underscored by differences in the way the risk of repeat violence is perceived by victims of IPV and victims' advocates entrusted with the task of conducting risk assessment and formulating safety plans as well as gathering information for the court case and providing whatever advocacy services are required (Cattaneo, 2007). In this study, HCSs' qualitative responses showed that they were aware of different types of intimate partner violence as well as aware of similarities and differences in their own and their parents' perceptions of IPV. Ironically, they appeared to be less aware of their own experiences as victims and/or perpetrators of IPV. Consequences may be dire if IPV advocates' perceptions and focus does not parallel with that of the victims and/or perpetrators of IPV. In Cattaneo's (2007) study, the victims of IPV based their assessment of risk on subtle and subjective perceptions, while the advocates gave priority to impersonal factors. The dichotomy is not surprising, but it highlights the need for more extensive research, especially into how one experience IPV perceive risk. A. R. Roberts (2007) viewed the woman's safety as the paramount concern. Neither the women nor the advocates were highly accurate in predicting future abuse. A model synthesizing complementary viewpoints with greater attention to the woman's perceptions might produce more accurate risk assessment.

According to Cattaneo (2007), there is compelling empirical evidence that women who experience IPV can accurately appraise their risk of future violent or nonviolent abuse. This was especially evident in A. R. Roberts' (2007) portrayal of women exposed to chronic abuse that followed a predictable pattern. Many women become attuned to 
subtle cues such as shifts in posture, voice, or facial expressions that warn of impending abuse (Cattaneo, 2007). This should not be surprising given that being aware of immanent violence is a survival skill for women in abusive relationships. From the professional standpoint, there is a debate over the utility of psychometrically validated instruments versus clinician assessment at risk. While some experts favor relying entirely on instruments, there is some evidence that clinicians base their judgments on factors other than empirically validated risk factors. Victim advocates gather abundant information to include under the heading of risk assessment in their intake protocols. However, it is not generally known what information they actually use to make their assessment.

\section{Limitations}

Cuevas and colleagues (2010) criticized the existing research on IPV among Latina/os for methodological weaknesses such as small sample size and reliance on only one type of violence. This study examined various forms of domestic violence, but findings are admittedly limited by the small sample size, which limits generalizability. HCSs may have trepidations of being exposed in academic settings regardless of confidentiality and may have altered their answers. In addition, the participants were recruited from two universities that both serve very diverse populations and less acculturated youths may have not been recruited. Although one of the two universities is a Hispanic serving institution, the lack of information about sub ethnic groups may pose bias in data collection. In addition, the participants might not be familiar with the style of questions on the survey questionnaires, and their responses might be inaccurate due to recall bias. The sample was mostly women which is also a limitation. 
Qualitative research emphasizes understanding individual perspectives as multiple truths and aims to aggregate the beliefs, social behavior, and processes that arise from participant perspectives and do not use the same practices or methods as with quantitative research (Prowse and Camfield, 2013). Participants' views were not collected as surveyed HCSs were not individually interviewed. The small number of questions included in the qualitative portion of the study was also a limitation.

\section{Conclusion}

This mixed methods study explored the perceptions and experiences of IPV among Hispanic young adult college students who attended two south Florida universities. The most notable finding was the pronounced discrepancy between the participants' perceived experience of IPV and their responses to the verbal aggression and sexual coercion subscales of the quantitative CTS2 instrument. Although the participants were aware that IPV can take many forms, milder forms of non-physical violence were not necessarily perceived as IPV. One reason for this may have been that verbal aggression that was not blatantly denigrating or belittling was common enough to be considered normal. Some types of sexual violence were also considered normal behavior, based on the qualitative responses, and roughly one-third of the group acknowledged using sexual coercion, interestingly equally by both genders.

The vast majority of the participants rejected traditional gender roles, and most participants were not highly religious. In addition, women and men were equally likely to be the perpetrators or victims of IPV. These findings did not support stereotypical assumptions about men and women of Latin heritage. There was some support for the theory of interpersonal transmission of violence, which appears to be stronger for 
victimization than perpetration. Whereas parental aggression was significantly linked with the level of IPV victimization, there was no parallel association between parental aggressions on IPV perpetration.

The overall findings suggest a need for additional exploration of the ways Hispanic college students perceive and experience IPV. Insight gained from qualitative exploration could be used to create and test a questionnaire that is specifically developed for this population group, and the knowledge gained from both types of research can be used to design appropriate and effective programs and interventions for addressing dating and relationship violence, which is increasingly recognized as a serious problem on college campuses. 


\section{LIST OF REFERENCES}

Ahrens, C. E., Rios-Mandel, L. C., Isas, L., \& del Carmen-Lopez, M. (2010). Talking about interpersonal violence: Cultural influences on Latinas' identification and disclosure of sexual assault and intimate partner violence. Psychological Trauma: Theory, Research, Practice, and Policy, 2, 284-295. doi:10.1037/a0018605

Allen, J. G. (2001). Traumatic relationships and serious mental disorders. New York, NY: Wiley.

American Psychiatric Association. (2000). Diagnostic and statistical manual of mental disorders (4th ed.). Washington, DC: Author.

Archer, J., \& Haigh, A. (1997). Do beliefs about aggression predict self-reported levels of aggression. British Journal of Social Psychology, 36, 83-105.

Bandura, A. (1977). Social learning theory. Englewood Cliffs, NJ: Prentice Hall.

Bandura, A. (1986). Social foundations of thought and action. Engelwood Cliffs, NJ: Prentice-Hall.

Bassuk, E., Dawson, R., \& Huntington, N. (2006). Intimate partner violence in extremely poor women: Longitudinal patterns and risk markers. Journal of Family Violence, 21, 387-399. doi:10.1007/s10896-006-9035-1

Belknap, R. A., \& Cruz, N. (2007). When I was in my home I suffered a lot: Mexican American women's descriptions of abuse in family of origin. Health Care for Women International, 28, 506-522. doi:10.1080/07399330701226545

Belsey, B. (2008). Cyberbullying. Retrieved from http://www.cyberbullying.org.

Bem, S. L. (1974). The measurement of psychological androgyny. Journal of Consulting and Clinical Psychology, 42, 155-162. doi:10.1177/0886260509340539

Bent-Goodley, T.B. (2004). Perceptions of domestic violence: A dialogue with African American women. Health \& Social Work, 29, 307-316.

Berg, S. (1988) Snowball sampling, in Kotz, S. and Johnson, N. L. (Eds.) Encyclopaedia of Statistical Sciences Vol. 8.

Black, M. C., Basile, K. C., Breiding, M. J., Smith, S. G., Walters, M. L., Merrick, M. T., ... Stevens, M. R. (2011). The national intimate partner and sexual violence survey (NISVS): 2010 summary report. Retrieved from http://www.cdc.gov /ViolencePrevention/pdf/NISVS_Report2010-a.pdf 
Bonomi, A. E., Anderson, M. L., Cannon, E. A., Siesnick, N., \& Rodriguez, M. A. (2008). Intimate partner violence in Latina and non-Latina women. American Journal of Preventive Medicine, 36, 43-48. doi:10.1016/j.amepre.2008.09.027

Bracero, W. (1998). Intimidades: Confianza, gender, and hierarchy in the construction of Latino-Latina therapeutic relationships. Cultural Diversity and Mental Health, 4, 264-277. doi:10.1037/1099-9809.4.4.264

Break the Cycle. (2005). Empowering youth to end domestic violence. Retrieved from http://www.breakthecycle.org/

Burns, N., \& Groves, S. K. (2005). The practice of nursing research: Conduct, critique, and utilization (5th ed.). Philadelphia, PA: Saunders.

Cambridge Dictionary Online. (2012a). Perpetrate. Retrieved from http://dictionary .cambridge.org/dictionary/british/perpetrate

Cambridge Dictionary Online. (2012b). Victimize. Retrieved from http://dictionary .cambridge.org/dictionary/british/victimize?q=victimization

Catalano, S. (2007). Intimate partner violence in the United States. Retrieved from http:// www.ojp.usdoj.gov/bjs/pub/pdf/ipvus.pdf

Cattaneo, L. B. (2007). Contributors to assessments of risk in intimate partner violence: How victims and professionals differ. Journal of Community Psychology, 35, 5775. doi:10.1002/jcop.20134

Centers for Disease Control and Prevention. (2006). Physical dating violence among high school students-United States, 2003. Morbidity and Mortality Weekly Report, 55, 532-535. Retrieved from http://www.cdc.gov/mmwr/PDF/wk/mm5519.pdf \#pagemode $=$ bookmarks\&page $=4$

Centers for Disease Control and Prevention. (2011). Division of Violence Prevention, Centers for Disease Control and Prevention. Understanding Intimate Partner Violence. Atlanta, GA: 2011. Available from http://www.cdc.gov/violenceprevention/pdf/IPV_factsheet-a.pdf

Clark, A. M. (1998). The qualitative and quantitative debate: Moving from positivism and confrontation to post-positivism and reconciliation. Journal of Advanced Nursing, 27, 1242-1249. doi:10.1046/j.1365-2648.1998.00651.x

Cochran J., Beeghley L., \& Bock E. (1988). Religiosity and alcohol behavior: An exploration of reference group theory. Sociological Forum, 3, 256-277. 
Cohen, J. (1988). Statistical power analysis for the behavioral sciences (2nd ed.). New York, NY: Academic.

Coker, A. L., Sanderson, M., Cantu, E., Huerta, D., \& Fadden, M. K. (2008). Frequency and types of partner violence among Mexican American college women. Journal of American College Health, 56, 665-673. doi:10.3200/JACH.56.6.665-674

Corvo, K. (2006). Violence, separation, and loss in the families of origin of domestically violent men. Journal of Family Violence, 21, 117-125. doi:10.1007/s10896-005 $-9011-1$

Crane, P. A., \& Constantino, R. E. (2003). Use of the Interpersonal Support Evaluation List (ISEL) to guide intervention development with women experiencing abuse. Issues in Mental Health Nursing, 24, 523-541. doi:10.1080/01612840305286

Creswell, J.W. (2003). Research design: Qualitative, quantitatative, and mix method approaches. Thousand Oaks, CA: Sage.

Creswell, J. W., \& Plano Clark, V. L. (2011). Designing and conducting mixed methods research (2nd ed.). Thousand Oaks, CA: Sage.

Cuevas, C. A., Sabina, C., \& Picard, E. H. (2010). Interpersonal victimization patterns and psychopathology among Latino women: Results from the SALAS study. Psychological Trauma: Theory, Research, Practice, and Policy 2, 296-306. doi:10.1037/a0020099

Cummings, A. M., Gonzalez-Guarda R. M., \& Sandoval, M. F. (2013). Intimate partner violence among Hispanics: A review of the literature. Journal of Family Violence 28:153-171. doi:10.1007/s10896-012-9478-5

Daley, E. M., \& Noland, V. J. (2001). Intimate partner violence in college students: A cross-cultural comparison. International Electronic Journal of Health Education, 4, 35-40. Retrieved from http://www.aahperd.org/aahe/publications/iejhe/loader .cfm? $\operatorname{csModule}=$ security/getfile\&pageid $=39132$

Daly, C. C. (2005). Definition of terms: Spirituality versus religiousness. Southern Medical Journal, 98, 1238-1239. doi:10.1097/01.smj.0000190594.43768.fc

Davidson, J. K., Moore, N. B., \& Ullstrup, K. M. (2004). Religiosity and sexual responsibility: Relationships of choice. American Journal of Health Behavior, 28, $335-346$. 
DeJonghe, E. S., Bogat, G. A., Levendosky, A. A., \& von Eye, A. (2008). Women survivors of intimate partner violence and post-traumatic stress disorder: Prediction and prevention. Journal of Postgraduate Medicine, 54, 294-300. doi:10.4103/0022-3859.41435

Dockterman, D. (2011). Country of origin profiles. Retrieved from http://pewhispanic.org /data/origins/

Edelson, M. G., Hokoda, A., \& Ramos-Lira, L. (2007). Differences in effects of domestic violence between Latina and non-Latina women. Journal of Family Violence, 22, 1-10. doi:10.1007/s10896-006-9051-1

Fagan, A. A. (2005). The relationship between adolescent physical abuse and criminal offending: Support for an enduring and generalized cycle of violence. Journal of Family Violence, 20, 279-290. doi:10.1007/s10896-005-6604-7

Fain, J. (2004). Reading, understanding, and applying nursing research (2nd ed.). Philadelphia, PA: F. A. Davis Company.

Faul, F., Erdfelder, E., Lang, A. G., \& Buchner, A. (2007). G*Power 3: A flexible statistical power analysis for the social, behavioral, and biomedical sciences. Behavior Research Methods, 39, 175-191.

Flake, D. F., \& Forste, R. (2006). Fighting families: Family characteristics associated with domestic violence in five Latin American countries. Journal of Family Violence, 17, 363-375. doi:10.1007/s10896-005-9002-2

Flores-Ortiz, Y. (1993). La mujer y la violencia: A culturally based model for the understanding and treatment of domestic violence in Chicana/Latina communities. In N. Alarcon, R. Castro, E. Perez, B. Pesquera, A. S. Riddell, \& P. Zavella, (Eds.), Chicana critical issues (pp. 169-182). Berkeley, CA: Third Woman Press.

Florida International University. (n.d.). CAPS. Victim empowerment program. Division of student affairs. Retieved from http://advocacy.fiu.edu/Services/Pages/default.aspx

Ford-Gilboe, M., Campbell, J., \& Berman, H. (1995). Stories and numbers: Coexistence without compromise. Advances in Nursing Science, 18, 14-26.

Fosco, G. M., DeBoard, R.L. \& Grych, J. H. (2007). Making sense of family violence: Implications of children's appraisals of interparental aggression for their shortand long-term functioning. European Psychologist, 12, 6-16. 
Foshee, V. A., Karriker-Jaffe, K. J., Reyes, H. L. M., Ennett, S. T., Suchindran, C., Bauman, K. E., \& Benefield, T. S. (2008). What accounts for demographic differences in trajectories of adolescent dating violence? An examination of intrapersonal and contextual mediators. Journal of Adolescent Health, 42, 596604.

Fraser, I. M., McNutt, L. A., Clark, C., Williams-Muhammed, D., \& Lee, R. (2002). Social support choices for help with abusive relationships: Perceptions of African American women. Journal of Family Violence, 17, 363-375. doi:10.1023/A :1020322600490

Glass, N., Perrin, N., Hanson, G., Bloom, T., Gardner, E., \& Campbell, J. C. (2008). Risk for reassault in abusive female same-sex relationships. American Journal of Public Health, 98, 1021-1027. doi:10.2105/AJPH.2007.117770

Goldstein, R. (1989). Power and sample size via MS/PC-DOS computers. American Statistician, 43, 253-262.

Gonzalez-Guarda, R. M., Peragallo, N., Vasquez, E. P., Urrutia, M. T., \& Mitrani, V. B. (2009). Intimate partner violence, depression, and resource availability among a community sample of Hispanic women. Issues in Mental Health Nursing, 30, 227-236. doi:10.1080/01612840802701109

Graham-Bermann, S. A., \& Brescoll, V. (2000). Gender, power, and violence: Assessing the family stereotypes of the children of batterers. Journal of Family Psychology, $14,600-612$.

Graham-Kevan, N., \& Archer, J. (2008). Does controlling behavior predict physical aggression and violence to partners? Journal of Family Violence, 23, 539-548. doi:10.1007/s10896-008-9162-y

Geertz, C. (1973). Interpretations of culture. New York, NY: Basic Books.

Haj-Yahia, M. M. (2003). Beliefs about wife beatings among Arab men from Israel: The influence of their patriarchal ideology. Journal of Family Violence, 18, 193-206. doi:10.1023/A:1024012229984

Harris, R. J., Firestone, J. M., \& Vega, W. A. (2005). The interaction of country of origin, acculturation, and gender role ideology on wife abuse. Social Science Quarterly, 86, 463-483. doi:10.1111/j.0038-4941.2005.00313.x

Hazen, A., \& Soriano, F. I. (2005). Experience of intimate partner violence among U.S. born, immigrant and migrant Latinas (National Institute of Justice Grant No: 2000-WT-VX-0017). Retrieved from http://www.ncjrs.gov/pdffiles1/nij/grants /211509.pdf 
Higginbotham, B. J., Ketring, S. A., Hibbert, J., Wright, D. W., \& Guarino, A. (2007). Relationship religiosity, adult attachment styles, and courtship violence experienced by females. Journal of Family Violence, 22, 55-62. doi:10.1007 /s10896-006-9049-8

Hirschi, T. (1969). The causes of delinquency. Los Angeles, CA: University of California Press.

Holsti, O.R. (1968). Content analysis. In G. Lindzey \& E. Aaronson (Eds.). The Handbook of Social Psychology. Reading, MA: Addison-Wesley.

Huff, R., \& Kline, M. (Eds.). (1999). Promoting health in multicultural populations. Thousand Oaks: Sage Publications.

Humphreys, J., \& Campbell, J. C. (Eds.). (2011). Family violence and nursing practice (2nd ed.). New York, NY: Springer.

Huth-Bocks, A. C., \& Hughes, H. M. (2008). Parenting stress, parenting behavior, and children's adjustment in families experiencing intimate partner violence. Journal of Family Violence, 23, 243-251.

Jakupcak, M., Lisak, D., \& Roemer, L. (2002). The role of masculine ideology and masculine gender role stress in men's perpetration of relationship violence. Psychology of Men and Masculinity, 3, 97-106. doi:10.1037/1524-9220.3.2.97

Johnson, M. P. (1995). Intimate terrorism and common couple violence: Two forms of violence against women. Journal of Marriage and the Family, 57, 283-294.

Jouriles, E. N., Spiller, L. C., Stephens, N., McDonald, R., \& Swank, P. (2000). Variability in adjustment of children of battered women: The role of child appraisals of interparent conflict. Cognitive Therapy and Research, 24, 233-249.

Kasturirangan, A., \& Williams, E. N. (2003). Counseling Latina battered women: A qualitative study of the Latina perspective. Journal of Multicultural Counseling and Development, 21, 162-178.

Kaufman, J., \& Zigler, E. (1987). Do abused children become abusive parents? American Journal of Orthopsychiatry, 57, 186-192.

Kaufman, J., \& Zigler, E. (1993). The intergenerational transmission of abuse is overstated. In R. J. Gelles \& D. R. Loseke (Eds.), Current controversies on family violence (pp. 209-221). Newbury Park, CA: Sage. 
Kline, P. (1999). Handbook of psychological testing (2nd ed.). New York, NY: Routledge.

Leason, K. (2005). They hear every insult, punch and slap. Community Care, 15-09-05, $34-35$.

Lee, E. (2007). Domestic violence and risk factors among Korean immigrant women in the United States. Journal of Family Violence, 22, 141-149. doi:10.1007/s10896 $-007-9063-5$

Lehrer, J. A., Lehrer, E. L., \& Zhao, Z. (2010). Physical dating violence victimization in college women in Chile. Journal of Women's Health, 19, 893-902. doi:10.1089 /jwh.2009.1583

Levitt, H. M., \& Ware, K. N. (2006). Religious leaders' perspectives on marriage, divorce, and intimate partner violence. Psychology of Women Quarterly, 30, 212 222. doi:10.1111/j.1471-6402.2006.00283.x

Lincoln, Y., \& Guba, E. (1985). Naturalistic inquiry. Beverly Hills, CA: Sage.

LoBiondo-Wood, G., \& Haber, J. (2006). Nursing research: Methods and critical appraisal for evidence-based practice (6th ed.). St. Louis, MO: Mosby Elsevier.

Lown, E. A., \& Vega, W. A. (2001). Prevalence and predictors of physical partner abuse among Mexican American women. American Journal of Public Health, 91, 441445 .

Lucente, S. W., Fals-Stewart, W., Richards, H. J., \& Goscha, J. (2001). Factor structure and reliability of the revised Conflict Tactics Scale for incarcerated female substance abusers. Journal of Family Violence, 16, 437-450. doi:10.1023/A :1012281027999

Lutenbacher, M., Cohen, A., \& Mitzel, J. (2003). Do we really help? Perspectives of abused women. Public Health Nursing, 20, 56-64.

Magdol, L., Moffitt, T. E., Caspi, A., \& Silva, P. A. (1998). Developmental antecedents of partner abuse: A prospective- longitudinal study. Journal of Abnormal Psychology, 107, 375-389.

Magnussen, L., Shoultz, J., Oneha, M. F., Hia, M. M., Brees-Saunders, Z., Akamine, M., \& Wong, E. (2004). Intimate-partner violence: A retrospective review of records in primary care settings. Journal of the American Academy of Nurse Practitioners, 16, 502-512. doi:10.1111/j.1745-7599.2004.tb00430.x 
Makepeace, J. M. (1981). Courtship violence among college students. Family Relations, $30,97-102$.

Malley-Morrison, K. \& Hines, D.A.. (2004). Family violence in a cultural perspective: Defining, understanding, and combating abuse. Thousand Oaks, CA: Sage.

Marín, G., Sabogal, F., VanOss Marín, B., Otero-Sabogal, R., \& Pérez-Stable, E. J. (1987). Development of a short acculturation scale for Hispanics. Hispanic Journal of Behavioral Sciences, 9, 183-205.

Marcus, N.E., Lindahl, K.M. \& Neena, N.M. (2001). Interparental conflict, children's social cognitions, and child aggression: A test of a mediational model. Journal of Family Psychology, 15 (2) 315-333.

McCloskey, L. A., Williams, C. M., Lichter, E., Gerber, M., Ganz, M. L., \& Sege, R. (2007). Abused women disclose partner interference with health care: An unrecognized form of battering. Journal of General Internal Medicine, 22, 10671072. doi:10.1007/s11606-007-0199-z

McDonald, R., Jouriles, E. N., Ramisetty-Mikler, S., Caetano, R., \& Green, C. E. (2006). Estimating the number of American children living in partner-violent families. Journal of Family Psychology, 20, 137-142. doi:10.1037/0893-3200.20.1.137

Mechanic, M. B., Weaver, T. L., \& Resick, P. A. (2000). Intimate partner violence and stalking behavior: Exploration of patterns and correlates in a sample of acutely battered women. Violence and Victims, 15, 55-72.

Merriam-Webster. (2011a). Hispanic. Retrieved from http://www.merriam-webster.com /dictionary/hispanic

Merriam-Webster. (2011b). Latino. Retrieved from http://www.merriam-webster.com /dictionary/latino?show=0\&t=1299510949

Merriam-Webster, (2011c). Patriarchy. Retrieved from http://www.merriam-webster .com/dictionary/patriarchy

Milletich, R. J., Kelley, M. L., Doane, A. N., \& Pearson, M. R. (2010). Exposure to interparental verbal and physical aggression and childhood physical and emotional abuse as related to physical aggression in undergraduate dating relationships. Journal of Family Violence, 25, 627-637.

Montalvo-Liendo, N. (2009). Cross-cultural factors in disclosure of intimate partner violence: An integrated review. Journal of Advanced Nursing, 65, 20-34. doi: 10.1111/J.1365-2648.2008.04850.X 
Montalvo-Liendo, N., Wardell, D. W., Englebretson, J., \& Reininger, B. M. (2009). Factors influencing disclosure of abuse by women of Mexican descent. Journal of Nursing Scholarship, 41, 359-367. doi:10.1111/j.1547-5069.2009.01304.x

Moore, T. M., \& Stuart, G. L. (2005). A review of the literature on masculinity and partner violence. Psychology of Men and Masculinity, 6, 46-61. doi:10.1037/ 1524-9220.6.1.46

Moretti, M. M., Obsuth, I., Odgers, C. L., \& Reebye, P. (2006). Exposure to maternal vs. paternal partner violence, PTSD, and aggression in adolescent girls and boys. Aggressive Behavior, 32, 385-395. doi:10.1002/ab.20137

Morse, J. M. (1991). Approaches to qualitative-quantitative methodological triangulation. Nursing Research, 40, 120-123.

Morse, J. (1994). Critical issues in qualitative research. Thousand Oaks, CA: Sage.

Murdaugh, C., Hunt, S., Sowell, R., \& Santana, I. (2004). Domestic violence among Hispanics in the southeastern United States: A survey and needs analysis. Journal of Family Violence, 21, 521-527. doi:10.1023/B:JOFV.0000019841 .58748 .51

Murrell, A. R., Christoff, K. A., \& Henning, K. R. (2007). Characteristics of domestic violence offenders: Associations with childhood exposure to violence. Journal of Family Violence, 22, 523-532. doi:10.1007/s10896-007-9100-4

Nam, Y., \& Tolman, R. (2002). Partner abuse and welfare receipt among African American and Latino women living in a low-income neighborhood. Social Work Research, 26, 241-251.

National Center for Injury Prevention and Control. (2003). Costs of intimate partner violence against women in the United States. Retrieved from http://www.cdc.gov /ncipc/pub-res/ipv_cost/ipv.htm

National Conference of State Legislature. (n.d.). Teen Violence. (N.p.). Retrieved from http://www.ncsl.org/research/health/teen-dating-violence.aspx

Noonan, R. K., \& Charles, D. (2009). Developing teen dating violence prevention strategies: Formative research with middle school youth. Violence Against Women, 15, 1087-1105. doi:10.1177/1077801209340761

Nunnally, J. C. (1978). Psychometric theory (2nd ed.). New York: McGraw-Hill. 
Panuzio, J., Taft, C. T., Black, D. A., Koenen, K. C., \& Murphy, C. M. (2007). Relationship abuse and victims' posttraumatic stress disorder symptoms: Associations with child behavior problems. Journal of Family Violence, 22, 177185.

Patton, M. (1990). Qualitative evaluation and research methods. Beverly Hills, CA: Sage.

Phinney, J. S. (2003). Ethnic identity and acculturation. In K. M. Chun, P. B. Organista, \& G. Marin (Eds.), Acculturation: Advances in theory, measurement, and applied research (pp. 63-81). Washington, DC: American Psychological Association.

Polit, D. F. (1996). Data analysis and statistics for nursing research. Stanford, CT: Appleton \& Lange.

Próspero, M. (2008). Effects of masculinity, sex, and control on different types of intimate partner violence perpetration. Journal of Family Violence, 23, 639-645. doi:10.1007/s10896-008-9186-3

Prowse, M. and Camfield, L. (2013). Improving the quality of development assistance: what role for qualitative methods in randomized trials. Progress in Development Studies. (13)1, 51-61.

Raffaelli, M., \& Ontai, L. L. (2004). Gender socialization in Latino/a families: Results from two retrospective studies. Sex Roles, 50, 287-299.

Ramirez, I. L. (2007). The relationship of acculturation and social integration to assaults on intimate partners among Mexican American and non-Mexican White students. Journal of Family Violence, 22, 533-542. doi:10.1007/S10896-007-9102-2

Ramos-Lira, L., Koss, M. P., \& Russo, N. F. (1999). Mexican American women's definitions of rape and sexual abuse. Hispanic Journal of Behavioral Sciences, 21, 236-265. doi:10.1117/0739986399213004

Risjord, M., Moloney, M., \& Dunbar, S. (2001). Methodological triangulation in nursing research. Philosophy of the Social Sciences, 31, 40-59. doi:10.1177 /004839310103100103

Roberts, A. R. (2007). Classification typology and assessment of five levels of woman battering. Journal of Family Violence, 21, 521-527. doi:10.1007/s10896-006 $-9044-0$

Roberts, T. A., Auinger, P., \& Klein, J. D. (2005). Intimate partner abuse and the reproductive health of sexually active female adolescents. Journal of Adolescent Health, 36, 380-385. doi:10.1016/j.jadohealth.2004.06.005 
Rodriguez, M. A., Sheldon, W. R., Bauer, H. M., \& Perez-Stable, E. J. (2001). The factors associated with disclosure of intimate partner abuse to clinicians. Journal of Family Practice, 50, 338-344.

Rondon, M. B. (2003). From Marianism to terrorism: The many faces of violence against women in Latin America. Archives of Women's Mental Health, 6, 157-163. doi:10.1007/s00737-003-0169-3

Roof, W. C. (1979). Concepts and indicators of religious commitment: A critical review. In R. Wuthnow (Ed.), The religious dimension (pp. 17-45). New York, NY: Academic Press.

Royston, P. (1992). Approximating the Shapiro-Wilk W-test for non-normality., Statistics and Computing. Retrieved from http://link.springer.com/article/10.1007/BF01891203\#page-1

Ruiz-Balsara, S. N. (2002). Gender belief systems and culture: The endorsement of machismo and marianismo by Hispanics/Latinos across gender, acculturation, education, socioeconomic, and religion categories. Dissertation Abstracts International, Section A. Humanities and Social Sciences, 62, 3199.

Ryder, A. G., Alden, L. E., \& Paulhus, D. L. (2000). Is acculturation unidimensional or bidimensional? A head-to-head comparison in the prediction of personality, selfidentity, and adjustment. Journal of Personality and Social Psychology, 79, 4965. doi:10.1037//0O22-3514.79.1.49

Sanderson, M., Coker, A. L., Roberts, R. E., Tortolero, S. R., \& Reininger, B. M. (2004). Acculturation, ethnic identity, and dating violence among Latino ninth-grade students. Preventive Medicine, 39, 373-383. doi:10.1016/j.ypmed.2004.01.034

Sansone, R. A., Chu, J., \& Wiederman, M. W. (2007). Self-inflicted bodily harm among victims of intimate-partner violence. Clinical Psychology and Psychotherapy, 14, 352-357. doi:10.1002/cpp.528

Schewe, P., Riger, S., Howard, A., Staggs, S. L., \& Mason, G. E. (2006). Factors associated with domestic violence and sexual assault victimization. Journal of Family Violence, 21, 469-475. doi:10.1007/s10896-006-9042-2

Schwartz, J., Hage, S., Bush, I., \& Burns, L. (2006). Unhealthy parenting and potential mediators as contributing factors to future intimate violence: A review of the literature. Trauma, Violence and Abuse, 7, 206-221. doi:10.1177 $/ 1524838006288932$ 
Shook, N. J., Gerrity, D. A., Jurich, J., \& Segrist, A. E. (2000). Courtship violence among college students: A comparison of verbally and physically abusive couples. Journal of Family Violence, 15, 1-22. doi: 10.1177/0886260509340543

Smith, B. A. M., Thompson, S., Tomaka, J., \& Buchanan, A. C. (2000). Development of the Intimate Partner Violence Attitude Scales (IPVAS) with a predominantly Mexican American college sample. Hispanic Journal of Behavioral Sciences, 27, 442-454. doi:10.1117/0739986305281233

Smith, M. (1990). Patriarchal ideology and wife beating: A test of feminist hypothesis. Violence and Victims, 5, 257-273.

Smith, M. E., \& Randall, E. J. (2007). Batterer intervention program: The victim's hope in ending the abuse and maintaining the relationship. Issues in Mental Health Nursing, 28, 1045-1063. doi:10.1080/01612840701522093

Snyder, I. B., Duncan, S. F., \& Larson, J. H. (2010). Assessing marriage education needs and interests among Latinos in a select western community. Journal of Comparative Family Studies, 41, 347-367.

Speziale, H. S., \& Carpenter, D. R. (2007). Qualitative research in nursing: Advancing the humanistic imperative (4th ed). Philadelphia, PA: Lippincott Williams \& Wilkins.

Steckler, A., McLeroy, K. R., Goodman, R. M., Bird, S. T., \& McCormick, L. (1992). Toward integrating qualitative and quantitative methods: An introduction. Health Education Quarterly, 19, 1-8. doi:10.1177/109019819201900101

Stets, J. E., \& Straus, M. A. (1992). The marriage license as a hitting license: A comparison of assaults in dating, cohabiting and married couples. In M. A. Straus \& R. J. Gelles (Eds.), Physical violence in families: Risk factors and adaptations to violence in 8,145 families (pp. 227-244). New Brunswick, NJ: Transaction.

Straus, M. A. (2004). Prevalence of violence against dating partners by male and female university students worldwide. Violence Against Women, 10, 790-811. doi:10 $.1177 / 1077801204265552$

Straus, M. A., Hamby, S. L., Boney-McCoy, S., \& Sugarman, D. B. (1995). The revised Conflict Tactics Scales. Durham, NH: Family Research Laboratory.

Straus, M. A., Hamby, S. L., Boney-McCoy, S., \& Sugarman, D. B. (1996).The revised Conflict Tactics Scales (CTS2): Development and preliminary psychometric data. Journal of Family Issues, 17, 283-316. 
Straus, M. A., Hamby, S. L., \& Warren, W. L. (2003). The Conflict Tactics Scales handbook. Los Angeles, CA: Western Psychological Services.

Straus, M. A., \& Ramirez, I. L. (2007). Gender symmetry in prevalence, severity, and chronicity of physical aggression against dating partners by university students in Mexico and USA. Aggressive Behavior, 33, 281-290. doi:10.1002/ab.20199

Sugihara, Y., \& Warner, J. A. (2002). Dominance and domestic abuse among Mexican Americans: Gender differences in the etiology of violence in intimate relationships. Journal of Family Violence, 17, 315-340. doi:10.1023/A :1020318532350

Thompson, M. P., Basile, K. C., Hertz, M. F., \& Sitterle, D. (2006). Measuring intimate partner violence victimization and perpetration: A compendium of assessment tools. Retrieved from http://www.cdc.gov/ncipc/dvp/compendium/IPV \%20Compendium.pdf

Tjaden, P., \& Thoennes, N. (2000). Full report of the prevalence, incidence, and consequences of violence against women: Findings from the National Violence Against Women Survey. Retrieved from http://www.ncjrs.gov/pdffiles1/nij /183781.pdf

Torres, S., \& Han, H. R. (2003). Women's perceptions of their male batterers' characteristics and level of violence. Issues in Mental Health Nursing, 24, 667679. doi:10.1080/01612840305317

Tower, L. E. (2003). Domestic violence screening: Education and institutional support correlates. Journal of Social Work Education, 39, 479-494.

Tower, L. E. (2006). Barriers in screening women for domestic violence: A survey of social workers, family practitioners, and obstetrician-gynecologists. Journal of Family Violence, 21, 245-257. doi:10.1007/s10896-006-9024-4

University of Miami School of Nursing and Health Studies. (n.d.). Center of Excellence for Health Disparities Research. El Centro. Retrieved from http://www.miami.edu/sonhs/index.php/elcentro/about_el_centro/

U.S. Census Bureau. (2010a). Facts for features: Hispanic heritage month September 15-October 15, 2010. Washington, DC: U.S. Department of Commerce. Retrieved from http://www.census.gov/newsroom/ releases/pdf/cb10ff-17 hispanic.pdf

U.S. Census Bureau. (2010b). State and county quickfacts: Hispanic origin. Retrieved from http://quickfacts.census.gov/qfd/meta/long_336231 
U.S. Department of Health and Human Services, Office of Disease Prevention and Health Promotion. (2010). Healthy people 2020. Retrieved from http://healthypeople .gov/2020/TopicsObjectives2020/pdfs/HP2020_brochure_with_LHI_508.pdf

U.S. Department of Justice. (n.d.). Grant Programs. N.p. (2013, December). Retrieved from http://www.ovw.usdoj.gov/ovwgrantprograms.htm\#1

Vandello, J. A., \& Cohen, D. (2003). Male honor and female fidelity: Implicit cultural scripts that perpetuate domestic violence. Journal of Personality and Social Psychology, 84, 997-1010. doi:10.1037/0022-3514.84.5.997

Vandello, J. A., Cohen, D., Grandon, R., \& Franiuk, R. (2009). Stand by your man: Indirect prescriptions for honorable violence and feminine loyalty in Canada, Chile, and the United States. Journal of Cross-Cultural Psychology, 40, 81-104. doi: $10.1117 / 0022022108326194$

"Victim Empowerment Program Mission Statement." Home. N.p.,(2013, August). Retrieved from http://advocacy.fiu.edu/About/Pages/default.aspx

Vogt, W. P. (1999) Dictionary of Statistics and Methodology: A Nontechnical Guide for the Social Sciences, London: Sage.

Wandrei, M. L., \& Rupert, P. A. (2000). Professional psychologists' conceptualizations of intimate partner violence. Psychotherapy, 37, 270-283. doi:10.1037/0033 $-3204.37 .3 .270$

Wasserman, C. (2004). Dating violence on campus: A fact of life. Networks, 16-19. Retrieved from_http://www.ncvc.org/ncve/AGP.Net/Components /documentViewer/Download.aspxnz?DocumentID=37929

Weis, L., Fine, M., Proweller, A., Bertram, C., \& Marusza, J. (1998). "I’ve slept in my clothes long enough": Excavating the sounds of domestic violence among women in the White working class. Urban Review, 30, 1-27.

World Health Organization. (2005). WHO multi-country study on women's health and domestic violence summary report of initial results on prevalence, health outcomes and women's responses. Geneva, Switzerland: Author. Retrieved from http://www.who.int/gender/violence/who_multicountry_study/summary_report /summary_report_English2.pdf

Wrangle J., Fisher J. W., \& Paranjape A. (2008). Ha sentido sola? Culturally competent screening for intimate partner violence in Latina women. Journal of Women's Health, 17, 261-268. doi:10.1089/jwh.2007.0394 


\section{APPENDICES}

APPENDIX A

CTS2 and CTS2-CA Limited License 
wpS

Western Psychological Services A Division of Manson Western Corporation

625 Alaska Avenue

Torrance, CA 90503 mww.wpspublish.com

May 1, 2013

Racquel Vera

Florida International University

10767 Lake Oak Way

Boca Raton, FL 33498

Re: Revised Conflict Tactics Scale (CTS2) and Revised Conflict Tactics Scale in a "Child-Adult" research application ("CTS2-CA")

Dear Ms. Vera -

WPS has processed your license for a specific adapted application of CTS2. By surface mail, you will soon receive a paid-in-full WPS invoice/receipt, which will serve as your license to a) adapt the format of the CTS2 for inclusion within a customized research survey, and to b) create and use an adaptation of the "ChildAdult" research version of the CTS2 (herein "CTS2-CA"), permitting adaptation, administration and scoring of the instruments up to two hundred and forty (240) times total (120 CTS2 uses; 120 "CTS2-CA" uses). This authorization is for sole use in your registered scholarly study, examining HCS perceptions and experiences of IPV focusing on levels of victimization and perpetration in relation to their gender roles, exposure to parental IPV, acculturation and religiosity that may contribute to or mitigate the incidence of IPV among HCSs - with no authorization for continued or commercial use - subject to the provisions of terms and conditions provided to you 20March'13.

With reference to condition (4) of WPS's March $20^{\text {th }}$ terms letter, please affix the following copyright notice in its entirety to each reprint of the CTS2 and "CTS2-CA":

Material from the CTS2 copyright (c) 2003 by Western Psychological Services. Adapted by R. Vera,

Florida International University, for specific, limited research use under license of the publisher, WPS,

625 Alaska Avenue, Torrance, California 90503, U.S.A. (rights@wpspublish.com). No additional

reproduction, in whole or in part, by any medium or for any purpose, may be made without the prior, written authorization of WPS. All rights reserved.

On behalf of WPS, I hope the CTS content well serves your study, and look forward in due course to learning of your research results.

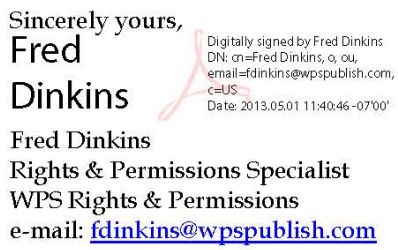

FD:sc 
wps

Western Psychological Services A Division of Manson Western Corporation 625 Alaska Avenue 625 Alaska Avenue
Torrance, CA 90503 mw.wpspublish.com

Dear Graduate Student:

Thank you for contacting Western Psychological Services for permission to reprint copyrighted test material within an appendix of your dissertation. When widely-distributed commercially produced tests are used, guidelines at most research universities do not call for inclusion of full instruments in thesis or dissertation volumes. In such cases, university policies are generally sensitive to the threat to commercial copyright and proprietary interests that is implicit in such copying or redistributing materials. The inclusion of instruments is generally limited to use of materials that are original to the dissertation author or that are otherwise unpublished and so might be considered difficult for subsequent readers to obtain.

As a publisher of formally developed test materials, WPS policy in such matters is to not authorize reprinting of our tests, subtests, or scales in their entirety, unless there is a committee requirement or other research-based reason that (1) requires you to reprint a test, subtest or scale in its entirety, and that (2) prevents the inclusion in your dissertation of original test forms. We can, as an alternative, readily provide authorization the reproduction of up to five representative sample items from the instrument upon receipt of your written request to that effect, including the specific item numbers desired for reprint. Also, if you need to reprint any other material from the test, including and not limited to material from the instrument's manual, please provide details by page, figure, table numbers, etc., for our consideration in authorizing inclusion of that material within your work.

If you need to pursue reprinting of the instrument in its entirety, please write again to WPS Rights and Permissions: Provide us with the reason you must reprint the subtests in their entirety (as opposed to selecting representative sample items); explain specifically why you are required to reproduce the original subtest (as opposed to binding an original protocol); and arrange for a supervising faculty member to co-sign the request. For expedience, please note that you may fax the letter to my attention at 424/201-6950, or have your professor e-mail it to me through his/her university e-mail address. For your additional reference in the event that your dissertation will be microfilmed, WPS will not authorize reproduction of our tests by microfilm, due to the public availability of the medium. While we regret any inconvenience our position may cause, we hope you appreciate our concern with ethical considerations.

We appreciate your interest in our material, as well as your consideration for its copyright. Please contact me if you have any questions.

Sincerely yours,

Susan Dunn Weinberg

WPS Rights and Permissions Manager

e-mail: weinberg@wpspublish.com

SDW:se 


\section{APPENDIX B}

Hispanic College Students' Relationship Study 


\section{INTRODUCTORY STATEMENT}

Before we start, there are a few things that I would like to go over with you.

The goal of this research study is to explore various relationship issues among college students who self-identifies as Hispanic or Latino/a. The survey includes some questions about your life, including questions about your family relations as well as about your partner (boy/girlfriend, significant other). Some of the questions will be personal; some may require you to take some time to think about them. I want to stress that there are no right or wrong answers to the questions that I will be asking. The most important thing is that you respond honestly. This information will be noted on the survey but everything you share to me will be strictly confidential.

It will take about 60 minutes to finish the survey. Take as much time as you need to answer any question. If you do not understand any of the questions, please ask me to explain it.

Do you have any questions before we begin?

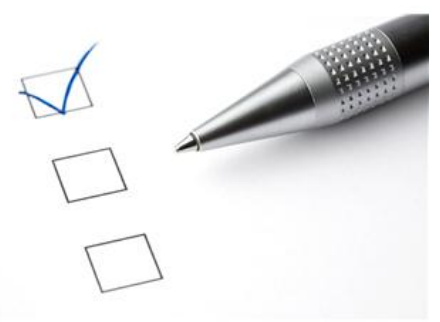

OK, let's start

\section{Participant ID\#}

\begin{tabular}{|l|l|l|l|l|l|}
\hline & & & & & \\
\hline 1 & 2 & 3 & 4 & 5 & 6 \\
\hline
\end{tabular}

- BOX $1 \& 2=$ Write the month of your birth [example: If you are born in January, write 0 \& 1; while for December, write $1 \& 2$ ].

- BOX $3 \& 4=$ Write the first two (2) letters or numbers of the street where you grew up.

- BOX 5 \& $6=$ Write the first two (2) letters of your mother's maiden name. 


\section{SECTION A: DEMOGRAPHIC QUESTIONS}

1.) How old are you? years old Gender: M |F [circle one]

Sexual Orientation [circle one]: Straight | Gay | Lesbian | Bi-sexual | Other:

2.) Where were you born?

Country

3.) What is your country of origin? (Circle one)
1. Mexico
2. Puerto Rico
3. Cuba
4. El Salvador
5. Dominican Republic
6. Guatemala
7. Colombia
8. Honduras
9. Ecuador
10. Peru
11. Other (please specify

4.) What is your religion?
1. Catholic
2. Christian
3. Jewish
4. Muslim
5. Other (please specify )

5.) In general, to what extent do you consider yourself religious?
1. Very religious
2. Religious
3. Somewhat religious
4. Not religious
5. Not at all religious

6.) In general, to what extent do you practice and adhere to laws and customs of your religion?
1. All the time
2. Most of the time
3. Sometimes 
4. Rarely

5. Never

7.) To what extent do you identify and feel affiliated with your religion?
1. All the time
2. Most of the time
3. Sometimes
4. Rarely
5. Never

8.) What language/s do you speak at home?
1. Spanish
2. English
3. Other:

9.) How long have you lived in the United States?
1.
2. Don't know/Unsure
3. Refused/No answer

10.) What was the reason you moved to the United States?

$\begin{array}{ll}\text { 1. } & \text { Education } \\ \text { 2. } & \text { Employment/Economic situation } \\ \text { 3. } & \text { Marriage } \\ \text { 4. } & \text { War/Political situation } \\ \text { 5. } & \text { Other/specify }\end{array}$

11.) What is your immigration status?
1. Green card (temporary)
2. Green Card (permanent)
3. US citizen
4. Undocumented
5. Political refugee/asylum seeker
6. $\quad$ Filing for papers
7. Other (please specify: student visa, etc. 
12.) Are you currently

1. Dating someone?

2. Have a boyfriend / girlfriend?

3. Not dating right now, but was in a relationship? [Skip to \#14]

13.) How long have you been in this relationship?

1. Less than 1 year

2. More than 1 year but less than 2 years

3. More than 2 years

14.) How long have you been separated?

1. Less than 1 year

2. More than 1 year but less than 2 years

3. More than 2 years

15.) Are you currently living with your partner?

1. Yes

2. No

16.) How old were you when you started dating? years old

17.) Did you experience problems with your partner while you are in this relationship?
1. Yes
2. No

18.) If yes, what problems did you experience?

1. Communication problems

2. Family problems

3. Mental Health problems

4. Children problems

5. Abuse problem

6. Sexual problems

7. Infidelity/Adultery

8. Jealousy and lack of trust

9. Financial problems

10. Other (please specify 
19.) When did the problems begin?

years months

20.) Do you live in a

1. Home?

2. College / university campus?

21.) Do you live with

1. Both parents?

2. Mother only?

3. Father only?

4. Relatives?

5. Friend(s)?

6. Other:

22.) In the past 12 months, how many people in your household have been physically hurt due to a fight or an argument?
1.
2. None
3. Don't know/Unsure
4. Refused/No answer

23.) If yes, is the person hurt a
1. Daughter
2. Son
3. Partner
4. Sister
5. Brother
6. Mother
7. Father
8. Yourself
9. Children
10. Other (please specify

24.) Are you currently working?
1. Yes
2. No [Skip to \#28]

25.) What is your job? 
26.) How many years/months have you been working?

__ years __ months

27.) What are your occupational/job skills?

28.) What is your individual yearly income, including financial aid, allowance, and other source of income? (Circle your best estimate)
1. None
2. Under $\$ 9,999$
3. $\$ 10,000$ to $\$ 19,999$
4. $\$ 20,000$ to $\$ 29,999$
5. $\$ 30,000$ to $\$ 39,999$
6. $\$ 40,000$ to $\$ 49,999$
7. $\$ 50,000$ to $\$ 59,999$
8. $\$ 60,000$ to $\$ 69,999$
9. $\$ 70,000$ to $\$ 79,999$
10. $\$ 80,000$ or more

29.) Are you currently financially dependent on your partner?

$\begin{array}{ll}\text { 1. } & \text { Yes } \\ \text { 2. } & \text { No }\end{array}$

30.) Who brings in the most money into your household?
1. Self
2. Partner
3. Parent
4. Welfare
5. Disability benefits
6. Relatives
7. Friends
8. Other:

31.) What kind of checking/savings account does your household have?

1. None

2. Separate accounts

3. Joint accounts

4. Partner only

5. Respondent only

6. Separate and joint account 


\section{Don't know/Unsure}

32.) Some people think that physical punishment (spanking, slapping, kicking, pinching) should be used to discipline children. Do you approve of parents to use physical punishment in disciplining their children?
1. Yes
2. No
3. Don't know/Unsure
4. Refused/No answer

33.) Have you ever received physical punishment in when you were younger?

1. Yes (please explain

2. No

3. Refused/No answer

\section{Please continue next page}




\section{SECTION B: Questions About How You Adopt the Beliefs and}

\section{Behaviors of Another Group or Culture [please circle]}

1. In general, what language(s) do you read and speak?

\begin{tabular}{c|c|c|c|c}
$\mathbf{1}$ & $\mathbf{2}$ & $\mathbf{3}$ & $\mathbf{4}$ & $\mathbf{5}$ \\
Only Spanish & $\begin{array}{c}\text { Spanish better } \\
\text { than English }\end{array}$ & Both equally & $\begin{array}{c}\text { English better } \\
\text { than Spanish }\end{array}$ & Only English
\end{tabular}

2. What was the language(s) you used as a child?

\begin{tabular}{c|c|c|c|c}
$\mathbf{1}$ & $\mathbf{2}$ & $\mathbf{3}$ & $\mathbf{4}$ & $\mathbf{5}$ \\
Only Spanish & $\begin{array}{c}\text { Spanish better } \\
\text { than English }\end{array}$ & Both equally & $\begin{array}{c}\text { English better } \\
\text { than Spanish }\end{array}$ & Only English
\end{tabular}

3. What language(s) do you usually speak at home?

\begin{tabular}{c|c|c|c|c}
$\mathbf{1}$ & $\mathbf{2}$ & $\mathbf{3}$ & $\mathbf{4}$ & $\mathbf{5}$ \\
Only Spanish & $\begin{array}{c}\text { Spanish better } \\
\text { than English }\end{array}$ & Both equally & $\begin{array}{c}\text { English better } \\
\text { than Spanish }\end{array}$ & Only English
\end{tabular}

4. In which language(s) do you usually think?

\begin{tabular}{c|c|c|c|c}
$\mathbf{1}$ & $\mathbf{2}$ & $\mathbf{3}$ & $\mathbf{4}$ & $\mathbf{5}$ \\
Only Spanish & $\begin{array}{c}\text { Spanish better } \\
\text { than English }\end{array}$ & Both equally & $\begin{array}{c}\text { English better } \\
\text { than Spanish }\end{array}$ & Only English
\end{tabular}

5. What language(s) do you usually speak with your friends?

\begin{tabular}{c|c|c|c|c}
$\mathbf{1}$ & $\mathbf{2}$ & $\mathbf{3}$ & $\mathbf{4}$ & $\mathbf{5}$ \\
Only Spanish & $\begin{array}{c}\text { Spanish better } \\
\text { than English }\end{array}$ & Both equally & $\begin{array}{c}\text { English better } \\
\text { than Spanish }\end{array}$ & Only English
\end{tabular}

6. In what language(s) are the T.V. programs you usually watch?

\begin{tabular}{c|c|c|c|c}
$\mathbf{1}$ & $\mathbf{2}$ & $\mathbf{3}$ & $\mathbf{4}$ & $\mathbf{5}$ \\
Only Spanish & $\begin{array}{c}\text { Spanish better } \\
\text { than English }\end{array}$ & Both equally & $\begin{array}{c}\text { English better } \\
\text { than Spanish }\end{array}$ & Only English
\end{tabular}


7. In what language(s) are the radio programs you usually listen to?

\begin{tabular}{c|c|c|c|c}
$\mathbf{1}$ & $\mathbf{2}$ & $\mathbf{3}$ & $\mathbf{4}$ & $\mathbf{5}$ \\
Only Spanish & $\begin{array}{c}\text { Spanish better } \\
\text { than English }\end{array}$ & Both equally & $\begin{array}{c}\text { English better } \\
\text { than Spanish }\end{array}$ & Only English
\end{tabular}

8. In general, in what language(s) are the movies, T.V. and radio programs you prefer to watch and listen to?

\begin{tabular}{c|c|c|c|c}
$\mathbf{1}$ & $\mathbf{2}$ & $\mathbf{3}$ & $\mathbf{4}$ & $\mathbf{5}$ \\
Only Spanish & $\begin{array}{c}\text { Spanish better } \\
\text { than English }\end{array}$ & Both equally & $\begin{array}{c}\text { English better } \\
\text { than Spanish }\end{array}$ & Only English
\end{tabular}

9. Your close friends are:

\begin{tabular}{|c|c|c|c|c|}
\hline 1 & 2 & 3 & 4 & 5 \\
\hline $\begin{array}{c}\text { All } \\
\text { atinos/Hispanics }\end{array}$ & $\begin{array}{l}\text { More Latinos } \\
\text { than Americans }\end{array}$ & $\begin{array}{c}\text { About half \& } \\
\text { half }\end{array}$ & $\begin{array}{c}\text { More Americans } \\
\text { than Latinos }\end{array}$ & $A \| A m$ \\
\hline
\end{tabular}

10. You prefer going to social gatherings/parties at which people are:

\begin{tabular}{c|c|c|c|c}
$\mathbf{1}$ & $\mathbf{2}$ & $\mathbf{3}$ & $\mathbf{4}$ & $\mathbf{5}$ \\
All & More Latinos & About half \& & More Americans & All Americans \\
Latinos/Hispanics & than Americans & half & than Latinos &
\end{tabular}

11. The persons you visit or who visit you are:

\begin{tabular}{c|c|c|c|c}
$\mathbf{1}$ & $\mathbf{2}$ & $\mathbf{3}$ & $\mathbf{4}$ & $\mathbf{5}$ \\
All & More Latinos & About half \& & $\begin{array}{c}\text { More Americans } \\
\text { than Latinos }\end{array}$ & All Americans \\
Latinos/Hispanics & than Americans & half & than
\end{tabular}

12. If you could choose your children's friends, you would want them to be:

\begin{tabular}{|c|c|c|c|c|}
\hline 1 & 2 & 3 & 4 & 5 \\
\hline $\begin{array}{c}\text { All } \\
\text { atinos/Hispanics }\end{array}$ & $\begin{array}{c}\text { More Latinos } \\
\text { than Americans }\end{array}$ & $\begin{array}{c}\text { About half \& } \\
\text { half }\end{array}$ & $\begin{array}{c}\text { More Americans } \\
\text { than Latinos }\end{array}$ & All Americans \\
\hline
\end{tabular}




\section{*SECTION C: Questions About You and Your Partner}

No matter how well a couple gets along, there are times when they disagree, get annoyed with one another, want different things from each other, or just have spats or fights because they are in a bad mood, are tired, or are upset for some other reason. Couples also have many different ways of trying to settle their differences. This is a list of things that might happen when you have differences. Some questions are about you and others are about your partner.

Please circle the response that describes how many times these things happened in the past year. If one of these things did not happen in the past year, but it happened before that, circle " $7 . "$

\section{How often did this happen in the past year?}

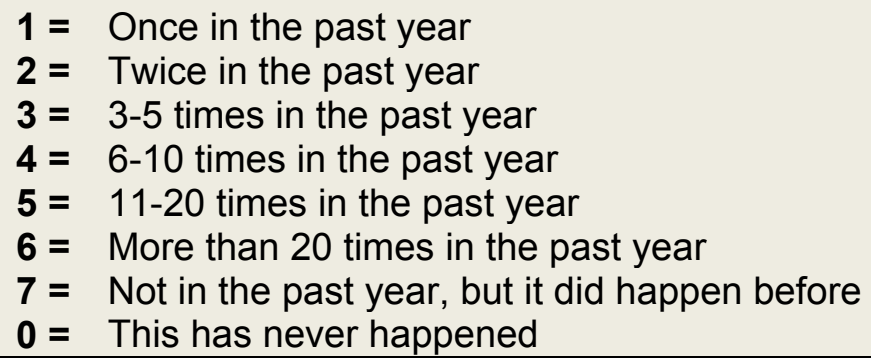

1. I showed my partner I cared even though we disagreed.

$$
\text { 1-----2------3-----4------5-----6------7-----0 }
$$

2. My partner showed care for me even though we disagreed.

$$
\text { 1-----2-----3-----4----------6------7----0 }
$$

3. I explained my side of a disagreement to my partner.

$$
\text { 1-----2-----3------4------5-----6------7-----0 }
$$

4. My partner explained his or her side of a disagreement to me.

$$
\text { 1-----2------3-----4------5------6-----7-----0 }
$$

5. I insulted or swore at my partner.

$$
\text { 1-----2------3-----4------5-----6-----7-----0 }
$$

6. My partner insulted or swore at me.

$$
\text { 1-----2------3-----4-----------6-----7-----0 }
$$


7. My partner threw something at me that could hurt.

$$
\text { 1-----2------3-----4------5-----6-----7-----0 }
$$

8. I twisted my partner's arm or hair.

$$
\text { 1-----2-----3-----4------5-----6-----7-----0 }
$$

9. My partner twisted my arm or hair.

$$
\text { 1-----2-----3------4-----5------6-----7-----0 }
$$

10. I had a sprain, bruise, or small cut because of a fight with my partner.

$$
1 \text {-----2-----3-----4-----5-----6-----7-----0 }
$$

11. My partner had a sprain, bruise, or small cut because of a fight with me.

$$
\text { 1-----2-----3-----4------5-----6-----7-----0 }
$$

12. I showed respect for my partner's feelings about an issue.

$$
\text { 1-----2------3-----4------5-----6-----7------0 }
$$

13. My partner showed respect for my feelings about an issue.

$$
\text { 1-----2------3-----4------5-----6-----7-----0 }
$$

14.I made my partner have sex without a condom.

$$
\text { 1-----2------3-----4------5-----6------7-----0 }
$$

15. My partner made me have sex without a condom.

$$
\text { 1-----2------3-----4------5-----6-----7-----0 }
$$

16.I pushed or shoved my partner.

$$
\text { 1-----2------3------4------5-----6------7-----0 }
$$

17. My partner pushed or shoved me.

$$
\text { 1-----2------3-----4------5-----6-----7-----0 }
$$


18. I used force (like hitting, holding down, or using a weapon) to make my partner have oral or anal sex.

$$
\text { 1-----2------3-----4------5-----6-----7-----0 }
$$

19. My partner used force to make me have oral or anal sex.

$$
\text { 1-----2------3-----4------5-----6-----7-----0 }
$$

20.I used a knife or gun on my partner.

$$
\text { 1-----2------3-----4------5-----6------7-----0 }
$$

21. My partner used a knife or gun on me.

$$
\text { 1-----2------3-----4------5-----6------7-----0 }
$$

22. I passed out from being hit on the head by my partner in a fight.

$$
\text { 1-----2------3-----4------5------6-----7-----0 }
$$

23. My partner passed out from being hit on the head by me in a fight.

$$
\text { 1-----2------3-----4------5-----6-----7-----0 }
$$

24. I called my partner fat or ugly.

$$
\text { 1-----2-----3-----4------5-----6-----7-----0 }
$$

25. My partner called me fat or ugly.

$$
\text { 1-----2------3-----4------5-----6------7-----0 }
$$


26. I punched or hit my partner with something that could hurt.

$$
\text { 1-----2------3-----4------5-----6-----7-----0 }
$$

27. My partner punched or hit me with something that could hurt.

$$
\text { 1-----2------3-----4------5-----6------7-----0 }
$$

28. I destroyed something belonging to my partner.

$$
\text { 1-----2------3-----4------5-----6------7-----0 }
$$

29. My partner destroyed something that belonged to me.

$$
\text { 1-----2-----3-----4------5-----6-----7-----0 }
$$

30.I went to a doctor because of a fight with my partner.

$$
\text { 1-----2------3-----4------5------6-----7-----0 }
$$

31. My partner went to a doctor because of a fight with me.

$$
\text { 1-----2-----3------4-----5------6-----7-----0 }
$$

32. I choked my partner.

$$
\text { 1-----2------3-----4------5-----6------7-----0 }
$$

33. My partner choked me.

$$
\text { 1-----2------3-----4------5-----6------7-----0 }
$$

34. I shouted or yelled at my partner.

$$
\text { 1-----2-----3------4------5-----6-----7-----0 }
$$

35. My partner shouted or yelled at me.

$$
\text { 1-----2------3-----4------5-----6-----7-----0 }
$$

36. I slammed my partner against a wall.

$$
\text { 1-----2------3-----4------5-----6-----7-----0 }
$$

37. My partner slammed me against a wall.

$$
\text { 1-----2------3-----4------5-----6-----7------0 }
$$




\begin{tabular}{|c|}
\hline $\begin{array}{c}\text { How often did this happen in the past year? } \\
\begin{array}{c}1=\text { Once that year } \mid \mathbf{2}=\text { Twice that year } \mid \mathbf{3}=3-5 \text { times that year } \mid \mathbf{4}=6-10 \\
\text { times that year } \mid \\
\mathbf{5}=11-20 \text { times that year } \mid \mathbf{6}=\text { More than } 20 \text { times that year } \mid \\
\mathbf{7}=\text { Not that year, but it did happened before or after that } \mid \mathbf{0}=\text { This never } \\
\text { happened }\end{array}\end{array}$ \\
\hline $\begin{array}{l}\text { 38. I said I was sure we could work out a problem. } \\
\qquad 1-----2-----3-----4-----5-----6-----7----0\end{array}$ \\
\hline 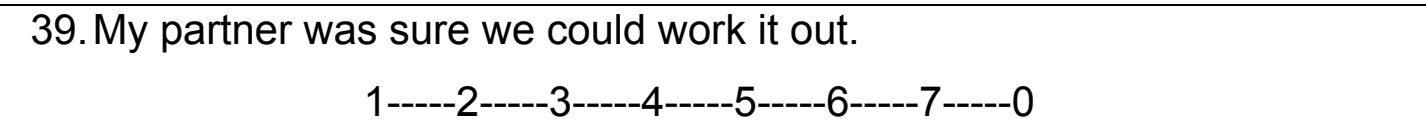 \\
\hline $\begin{array}{l}\text { 40.I needed to see a doctor because of a fight with my partner, but I didn't. } \\
\text { 1-----2-----3----4----5----6-----7----0 }\end{array}$ \\
\hline 41. My partner needed to see a doctor because of a fight with me, but didn't. \\
\hline $\begin{array}{l}\text { 42.I beat up my partner. } \\
\text { 1-----2-----3-----4-----5-----6-----7-----0 }\end{array}$ \\
\hline $\begin{array}{l}\text { 43. My partner beat me up. } \\
\qquad 1----2-----3----4----5----6-----7----0\end{array}$ \\
\hline $\begin{array}{l}\text { 44.I grabbed my partner. } \\
\qquad 1-----2-----3-----4-----5----6-----7-----0\end{array}$ \\
\hline $\begin{array}{l}\text { 45. My partner grabbed me. } \\
\qquad 1-----2-----3----4-----5-----6-----7----0\end{array}$ \\
\hline $\begin{array}{l}\text { 46. I used force (like hitting, holding down, or using a weapon) to make my } \\
\text { partner have sex. }\end{array}$ \\
\hline
\end{tabular}


47. My partner used force to make me have sex.

$$
\text { 1-----2------3-----4------5-----6-----7-----0 }
$$

48. I stomped out of the room or house or yard during a disagreement.

$$
1 \text {-----2------3-----4------5-----6-----7-----0 }
$$

49. My partner stomped out of the room or house or yard during a disagreement.

$$
\text { 1-----2-----3------4------5-----6------7-----0 }
$$

50.I insisted on sex when my partner did not want to (but did not use physical force).

$$
\text { 1-----2-----3-----4------5-----6------7-----0 }
$$

51. My partner insisted that I have sex when I didn't want to (but did not use physical force).

$$
\text { 1-----2-----3-----4-----5-----6-----7-----0 }
$$

52. I slapped my partner.

$$
\text { 1-----2------3-----4-----5------6-----7----0 }
$$

53. My partner slapped me.

$$
\text { 1-----2-----3-----4------5-----6-----7-----0 }
$$

54.I had a broken bone from a fight with my partner.

$$
\text { 1-----2-----3-----4------5-----6-----7-----0 }
$$

55. My partner had a broken bone from a fight with me.

$$
\text { 1-----2------3-----4------5-----6-----7-----0 }
$$

56. I used threats to make my partner have oral or anal sex.

$$
\text { 1-----2------3-----4------5-----6------7-----0 }
$$

57. My partner used threats to make me have oral or anal sex.

$$
\text { 1-----2------3-----4------5-----6-----7-----0 }
$$

58. I suggested a compromise to a disagreement.

$$
\text { 1-----2------3-----4-----5-----6-----7-----0 }
$$


59. My partner suggested a compromise to a disagreement.

$$
1 \text {-----2------3-----4------5------6-----7-----0 }
$$

60. I burned or scalded my partner on purpose.

$$
\text { 1-----2-----3------4------5-----6------7-----0 }
$$

61. My partner burned or scalded me on purpose.

$$
\text { 1-----2-----3------4-----5------6-----7-----0 }
$$

62.I insisted my partner have oral or anal sex (but did not use physical force).

$$
\text { 1-----2------3------4------5-----6------7-----0 }
$$

63. My partner insisted I have oral or anal sex (but did not use physical force).

$$
\text { 1-----2------3-----4------5-----6------7-----0 }
$$

64.I accused my partner of being a lousy lover.

$$
\text { 1-----2------3-----4------5-----6------7-----0 }
$$

65. My partner accused me of being a lousy lover.

$$
\text { 1-----2------3-----4------5------6-----7-----0 }
$$

66. I did something to spite my partner.

$$
\text { 1-----2------3-----4------5-----6-----7-----0 }
$$

67. My partner did something to spite me.

$$
\text { 1-----2------3-----4------5-----6-----7-----0 }
$$

68. I threatened to hit or throw something at my partner.

$$
\text { 1-----2-----3-----4----------6-----7-----0 }
$$

69. My partner threatened to hit or throw something at me.

$$
\text { 1-----2------3-----4-----5-----6-----7-----0 }
$$




\begin{tabular}{|c|}
\hline $\begin{array}{c}\text { How often did this happen in the past year? } \\
\begin{array}{c}\mathbf{1}=\text { Once that year } \mid \mathbf{2}=\text { Twice that year } \mid \mathbf{3}=3-5 \text { times that year } \mid \mathbf{4}=6-10 \\
\text { times that year } \mid \\
\mathbf{5}=11-20 \text { times that year } \mid \mathbf{6}=\text { More than } 20 \text { times that year } \mid \\
\mathbf{7}=\text { Not that year, but it did happened before or after that } \mid \mathbf{0}=\text { This never } \\
\text { happened }\end{array}\end{array}$ \\
\hline $\begin{array}{l}\text { 70.I felt physical pain that still hurt the next day because of a fight with my } \\
\text { partner. }\end{array}$ \\
\hline $\begin{array}{l}\text { 71. My partner still felt physical pain the next day because of a fight we had. } \\
\text { 1-----2-----3-----4-----5----6-----7-----0 }\end{array}$ \\
\hline $\begin{array}{l}\text { 72.I kicked my partner. } \\
\text { 1-----2-----3-----4-----5-----6-----7-----0 }\end{array}$ \\
\hline $\begin{array}{l}\text { 73. My partner kicked me. } \\
\text { 1-----2-----3-----4----5-----6-----7----0 }\end{array}$ \\
\hline
\end{tabular}

74.I used threats to make my partner have sex.

$$
\text { 1-----2-----3-----4------5-----6-----7-----0 }
$$

75. My partner used threats to make me have sex.

$$
\text { 1-----2------3-----4------5-----6-----7-----0 }
$$

76. I agreed to try a solution to a disagreement my partner suggested.

$$
\text { 1-----2------3-----4------5-----6-----7-----0 }
$$

77. My partner agreed to try a solution I suggested.

$$
\text { 1-----2-----3------4-----5-----6------7-----0 }
$$




\section{Please continue next page}

*Material from the CTS2 copyright (C) 2003 by Western Psychological Services. Adapted by R. Vera, Florida International University, for specific, limited research use under license of the publisher, WPS, 625 Alaska Avenue, Torrance, California 90503, U.S.A. (rights@wpspublish.com). No additional reproduction, in whole or in part, by any medium or for any purpose, may be made without the prior, written authorization of WPS. All rights reserved. 


\section{*SECTION D: Questions About Your Experiences With Your Parents}

Directions: No matter how parents get along, there are times when they disagree, get annoyed with each other, want different things from each other, just have spats or fights because they are in a bad mood, are tired, or for some other reasons. Parents also have many ways of trying to settle their differences with each other. This is a list of things that might happen when your parents had differences or were angry with each other.

Please circle how many times each of them did the things on the list in the year when you were about 13 years old. If a parent did not do one of these things in the year when you were 13 years old but happened some other year before or after that, circle "7".

How often did this happen in the year when you were about 13 years old?

$1=$ Once that year $\mid \mathbf{2}=$ Twice that year $\mid \mathbf{3}=3-5$ times that year $\mid$

$4=6-10$ times that year

$\mathbf{5}=11-20$ times that year $\mid \mathbf{6}=$ More than 20 times that year $\mid$

$7=$ Not that year, but it did happened before or after that $\mid \mathbf{0}=$ This never happened

1. Mother showed she cared about father even when they disagreed

123345667

2. Father showed he cared about mother even when they disagreed

$\begin{array}{llllllll}1 & 2 & 3 & 4 & 5 & 6 & 7 & 0\end{array}$

3. Mother explained her side of a disagreement to father

$\begin{array}{llllllll}1 & 2 & 3 & 4 & 5 & 6 & 7 & 0\end{array}$

4. Father explained his side of a disagreement to mother

5. Mother insulted or swore at father

12234456670

6. Father insulted or swore at mother

7. Mother threw something at father that could hurt

8. Father threw something at mother that could hurt

$\begin{array}{llllllll}1 & 2 & 3 & 4 & 5 & 6 & 7 & 0\end{array}$

$\begin{array}{llllllll}1 & 2 & 3 & 4 & 5 & 6 & 7 & 0\end{array}$

9. Mother twisted father's arm or hair

$\begin{array}{llllllll}1 & 2 & 3 & 4 & 5 & 6 & 7 & 0\end{array}$

10. Father twisted mother's arm or hair.

11. Mother had a sprain, bruise, or small cut because of a fight with father

123455670

12. Father had a sprain, bruise, or small cut because of a fight with mother

$\begin{array}{llllllll}1 & 2 & 3 & 4 & 5 & 6 & 7 & 0\end{array}$

13. Mother showed respect for father's feelings about an issue

$\begin{array}{llllllll}1 & 2 & 3 & 4 & 5 & 6 & 7 & 0\end{array}$

14. Father showed respect for mother's feelings about an issue

$\begin{array}{llllllll}1 & 2 & 3 & 4 & 5 & 6 & 7 & 0\end{array}$ 
How often did this happen in the year when you were about 13 years old?

$1=$ Once that year $\mid \mathbf{2}=$ Twice that year $\mid 3=3-5$ times that year $\mid 4=6-10$ times that year $\mid$

$5=11-20$ times that year $\mid \mathbf{6}=$ More than 20 times that year $\mid$

$7=$ Not that year, but it did happened before or after that $\mid \mathbf{0}=$ This never happened

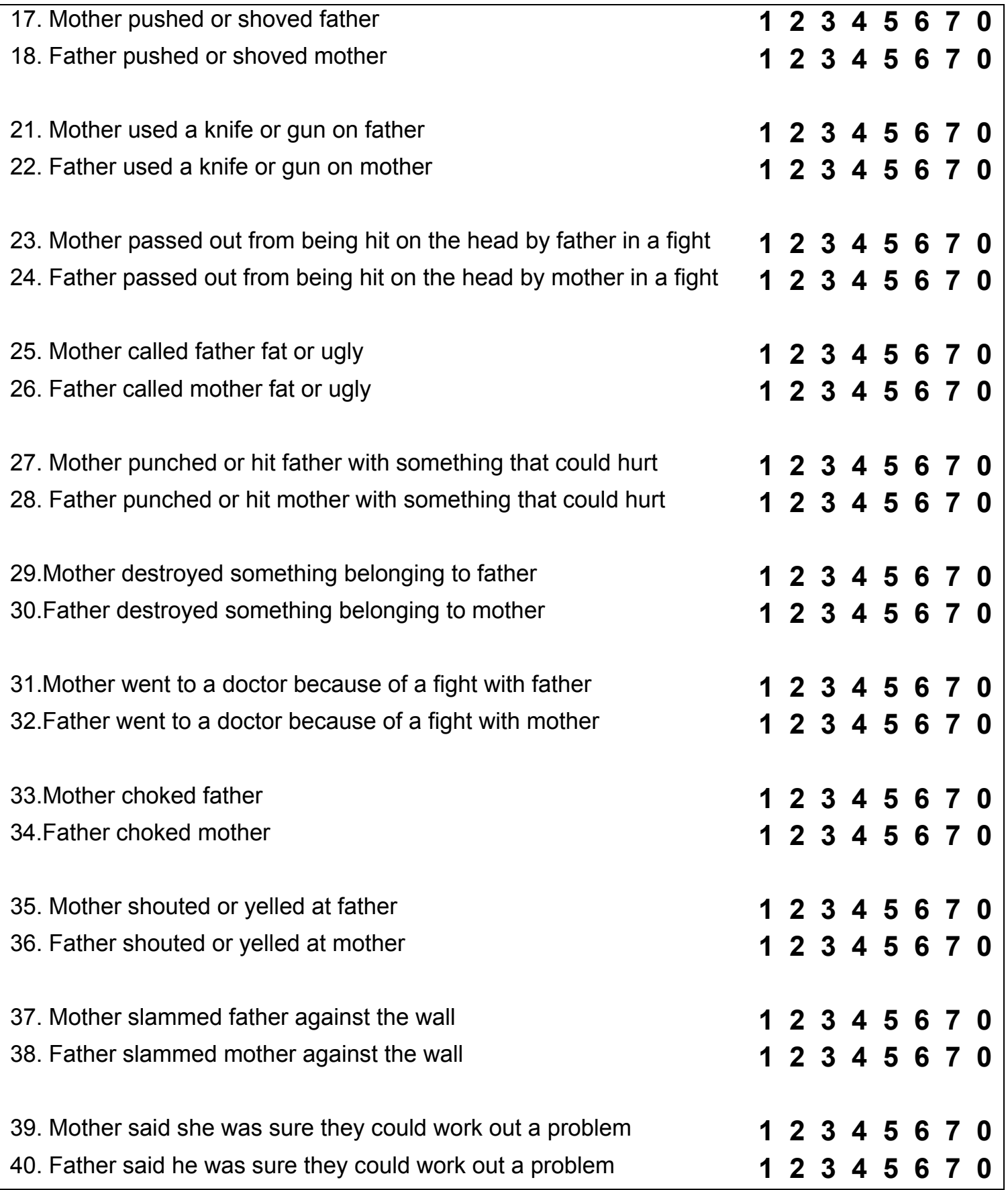


(Section D, continue)

How often did this happen in the year when you were about 13 years old?

$1=$ Once that year $\mid 2=$ Twice that year $\mid 3=3-5$ times that year $\mid 4=6-10$ times that year $\mid$

$5=11-20$ times that year $\mid \mathbf{6}=$ More than 20 times that year $\mid$

$7=$ Not that year, but it did happened before or after that $\mid 0=$ This never happened

41. Mother needed to see a doctor because of a fight with father, but $\quad \begin{array}{llllllll}1 & 2 & 3 & 4 & 6 & 7 & 0\end{array}$ didn't go.

42. Father needed to see a doctor because of a fight with father, but didn't go.

43. Mother beat up father

1233455676

44. Father beat up mother

12233455677

45. Mother grabbed father

12234456676

46. Father grabbed mother

12234456670

49. Mother stomped out of the room or house or yard when she had disagreement with father

50. Father stomped out of the room or house or yard when she had disagreement with mother

53. Mother slapped father

1223455670

54. Father slapped mother

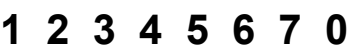

1233455677

55. Mother had a broken bone from a fight with father

1233455677

56. Father had a broken bone from a fight with mother

$\begin{array}{llllllll}1 & 2 & 3 & 4 & 5 & 6 & 7 & 0\end{array}$

59. Mother suggested a compromise to a disagreement with father

60. Father suggested a compromise to a disagreement with mother

12234456677

1233455677

61. Mother burned or scalded father on purpose

12234556770

62. Father burned or scalded mother on purpose

12234456670

67. Mother did something to spite father

1233456670

68. Father did something to spite mother

69. Mother threatened to hit or throw something at father

$\begin{array}{llllllll}1 & 2 & 3 & 4 & 5 & 6 & 7 & 0\end{array}$

70. Father threatened to hit or throw something at mother

$\begin{array}{llllllll}1 & 2 & 3 & 4 & 5 & 6 & 7 & 0\end{array}$ 
(Section D, continue)

How often did this happen in the year when you were about 13 years old?

$1=$ Once that year $\mid 2=$ Twice that year $\mid 3=3-5$ times that year $\mid 4=6-10$ times that year $\mid$

$5=11-20$ times that year $\mid \mathbf{6}=$ More than 20 times that year $\mid$

$7=$ Not that year, but it did happened before or after that $\mid \mathbf{0}=$ This never happened

71. Mother felt physical pain that still hurt the next day because of a fight with father

72. Father felt physical pain that still hurt the next day because of a

fight with mother

73. Mother kicked father

122334556770

74. Father kicked mother

12234456670

77. Mother agreed to try a solution to a disagreement suggested by father

78.Father agreed to try a solution to a disagreement suggested by mother

1233456670

1233456670

1233455676

12234556770

\section{Please continue next page}

*Material from the CTS2 copyright (C) 2003 by Western Psychological Services. Adapted by R. Vera, Florida International University, for specific, limited research use under license of the publisher, WPS, 625 Alaska Avenue, Torrance, California 90503, U.S.A. (rights@wpspublish.com). No additional reproduction, in whole or in part, by any medium or for any purpose, may be made without the prior, written authorization of WPS. All rights reserved. 
SECTION E: Questions About Your Beliefs on Men's Role in Women's Lives

We are interested in how you feel about the following statements. There is no right or wrong answers, only opinions. Tell us how you feel using the following scale:

$$
\begin{aligned}
& 1=\text { Strongly Agree } \\
& 2=\text { Agree } \\
& 3=\text { Undecided } \\
& 4=\text { Disagree } \\
& 5=\text { Strongly Disagree }
\end{aligned}
$$

A. A man has the right to decide whether or not his wife/partner/girlfriend should work outside the home.

\begin{tabular}{|ccccc|}
\hline $\begin{array}{c}\text { Strongly Agree } \\
1\end{array}$ & 2 & 3 & 4 & Strongly Disagree \\
\hline
\end{tabular}

B. A man has the right to decide whether his wife/partner/girlfriend should go out in the evening with her friends.

\begin{tabular}{|lcccc|}
\hline \multicolumn{2}{|l}{ Strongly Agree } & & & Strongly Disagree \\
1 & 2 & 3 & 4 & 5 \\
\hline
\end{tabular}

C. Sometimes it is important for a man to show his wife/partner/girlfriend that he is head of the house.

\begin{tabular}{|llllc|}
\hline \multicolumn{2}{|c}{ Strongly Agree } & & & Strongly Disagree \\
1 & 2 & 3 & 4 & 5 \\
\hline
\end{tabular}

D. A man has the right to have sex with his wife/partner/girlfriend when he wants, even though she may not want to.

\begin{tabular}{|lrllc|}
\hline Strongly Agree & & & Strongly Disagree \\
1 & 2 & 3 & 4 & 5 \\
\hline
\end{tabular}

E. Women should be protected by law if their partners beat them.

\begin{tabular}{|lrrrc|}
\hline \multicolumn{2}{|c}{ Strongly Agree } & & & Strongly Disagree \\
1 & 2 & 3 & 4 & 5 \\
\hline
\end{tabular}


Please check the appropriate box that you perceive applies to you:

YES NO

\section{QUESTION \#1:}

Do/did you consider yourself a victim/survivor of a partner or dating violence?

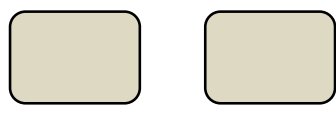

\section{QUESTION \#2:}

Do/did you consider yourself a perpetrator of violence while in a relationship?
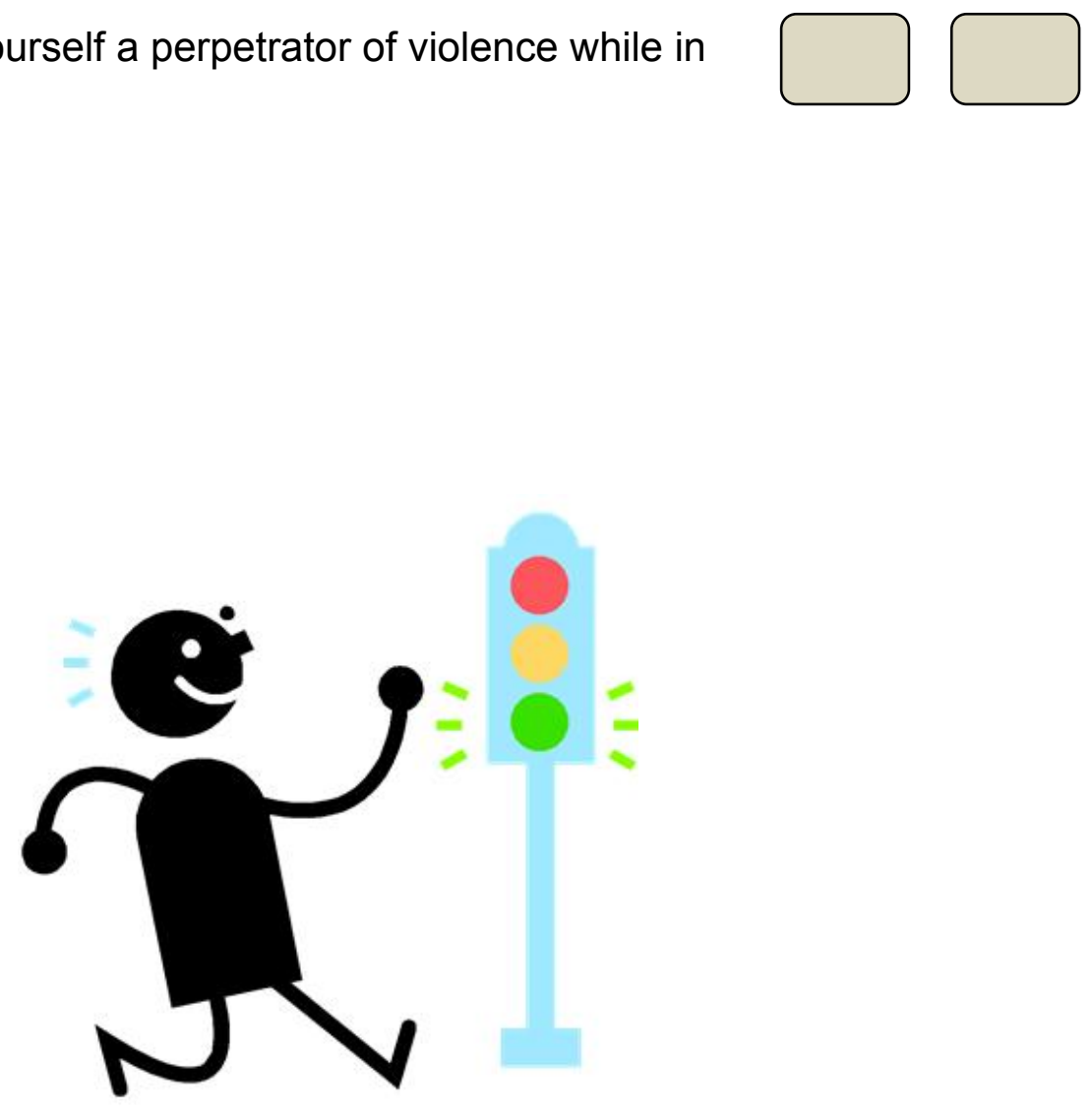

Please continue next page 
Based on Question \#1 and Question \#2, please answer the following openended questions to the best of your ability. Remember, there is no right or wrong answer.

\begin{tabular}{|l|l|l|}
\hline & $\begin{array}{l}\text { OPEN ENDED } \\
\text { QUESTIONS }\end{array}$ & ANSWERS \\
\hline a. & $\begin{array}{l}\text { What does intimate } \\
\text { partner violence mean to } \\
\text { you? } \\
\text { Please give at least one } \\
\text { example. }\end{array}$ & \\
\hline b. & $\begin{array}{l}\text { What do you think intimate } \\
\text { partner violence mean } \\
\text { your parents? } \\
\text { Please give at least one } \\
\text { example. }\end{array}$ \\
\hline
\end{tabular}




\begin{tabular}{|l|l|l|}
\hline $\begin{array}{l}\text { OPEN ENDED } \\
\text { QUESTIONS }\end{array}$ & ANSWERS \\
\hline c. & $\begin{array}{l}\text { Do you believe that your } \\
\text { definition of intimate } \\
\text { partner violence similar to } \\
\text { that of your parents? } \\
\text { Why? } \\
\begin{array}{l}\text { Please give at least one } \\
\text { example. }\end{array}\end{array}$ & \\
\hline d. & $\begin{array}{l}\text { Tell me about what kinds } \\
\text { of violence there are. }\end{array}$ & \\
\hline
\end{tabular}




$$
\text { The End. }
$$

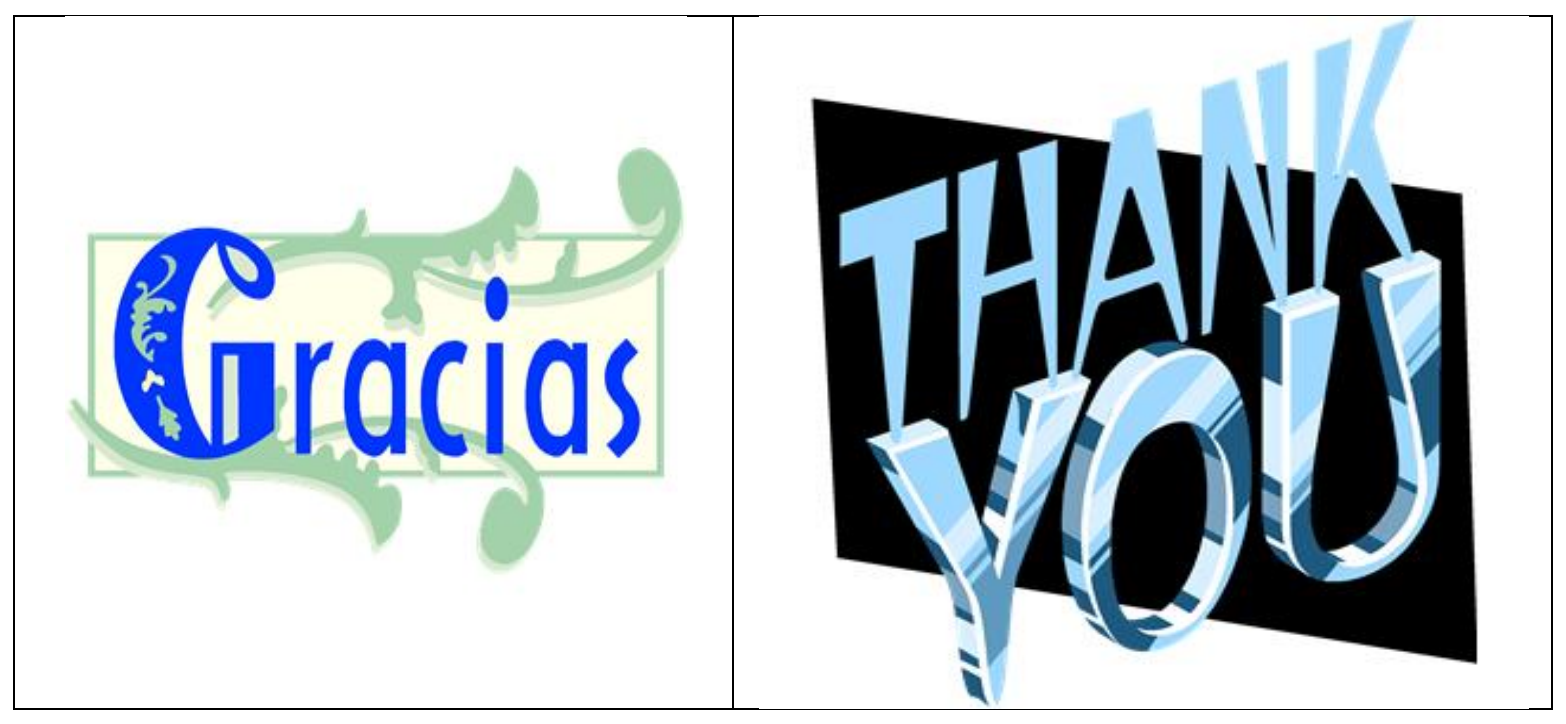


VITA

RACQUEL VERA

Born, Quezon City, Philippines

1991

B.S. in Nursing

United Doctors Medical Center

1994-1999

Staff Nurse, Charge Nurse

Jackson North Hospital (formerly Parkway

Regional Medical Center)

Miami, Florida

1997-1999

1998-2000

Staff Nurse

Joe DiMaggio Children's Hospital,

Hollywood, Florida

Children's Multidisciplinary Assessment

Team (CMAT) Coordinator - Broward

County

Children's Medical Services

Fort Lauderdale, Florida

2000

M.S.N. in Nursing

Florida International University

Miami, Florida

2000-2003

Pediatric Nurse Practitioner

Boca Raton, Florida

2003-Present

Assistant Professor

Community and Pediatric Clinical

Coordinator

College of Nursing, South University

West Palm Beach, Florida

2005-2008

On-line Nurse Faculty / Facilitator

College of Nursing, South University

West Palm Beach, Florida

2007-2008

Nurse Educator

Health Care District of Palm Beach County, Florida 
$2008-2010$

2013-Present

2013
Adjunct- Clinical Professor

College of Nursing and Health Sciences

Florida International University

Miami, Florida

RN-BSN Program Director

College of Nursing \& Public Health

South University

West Palm Beach, Florida

Ph.D. in Nursing Science

Florida International University

Miami, Florida

\section{PUBLICATIONS AND PRESENTATIONS}

Siegel, R. V. (July, 2010). Perceptions of intimate partner violence among Haitian

women: Poster presented at the 21st International Nursing Research Congress: Global

Diversity through Research, Education and Evidence-Based Practice. Orlando, Florida.

\section{PROFESSIONAL LICENSURE AND CERTIFICATION}

Florida, License Number RN/ARNP 2724872, Expiration Date July 30, 2014

Florida, Department of Health, Certified Immunization Nurse Adults and Pediatrics.

$\begin{array}{ll}\text { MEMBERSHIPS IN PROFESSIONAL ORGANIZATIONS } \\ 2001 \text { - present } & \begin{array}{l}\text { National Association for Pediatric Nurse Practitioners (NAPNAP) } \\ \text { Member }\end{array} \\ 2003 \text { - present } & \text { National League of Nursing (NLN) Member } \\ 2005 \text { - present } & \text { Florida Nurses Association (FNA) Member } \\ 2009-\text { present } & \text { Association of Women's Health, Obstetric and Neonatal Nurses } \\ & \text { (AWOHNN) Member } \\ 2009-\text { present } & \text { Sigma Theta Tau International - Pi Alpha (STTI) Member } \\ 2009-\text { present } & \text { Delta Epsilon Iota (DEI) Academic Honor Society Member } \\ 2009-\text { present } & \text { American Nurses Association (ANA) Member } \\ 2009-\text { present } & \text { Southern Research Nursing Society (SRNS) Member }\end{array}$

